\title{
Axiomatising Incomplete Preferences through Sets of Desirable Gambles
}

\author{
Marco Zaffalon \\ IDSIA \\ Galleria 2 \\ CH-6928 Manno (Lugano), Switzerland \\ Enrique Miranda \\ Department of Statistics and Operations Research \\ University of Oviedo \\ C-Federico García Lorca, 18 \\ 33007 Oviedo, Spain
}

MIRANDAENRIQUE@UNIOVI.ES

\begin{abstract}
We establish the equivalence of two very general theories: the first is the decisiontheoretic formalisation of incomplete preferences based on the mixture independence axiom; the second is the theory of coherent sets of desirable gambles (bounded variables) developed in the context of imprecise probability and extended here to vector-valued gambles. Such an equivalence allows us to analyse the theory of incomplete preferences from the point of view of desirability. Among other things, this leads us to uncover an unexpected and clarifying relation: that the notion of state independence - the traditional assumption that we can have separate models for beliefs (probabilities) and values (utilities) - coincides with that of strong independence in imprecise probability; this connection leads us also to propose much weaker, and arguably more realistic, notions of state independence. Then we simplify the treatment of complete beliefs and values by putting them on a more equal footing. We study the role of the Archimedean condition - which allows us to actually talk of expected utility - identify some weaknesses and propose alternatives that solve these. More generally speaking, we show that desirability is a valuable alternative foundation to preferences for decision theory that streamlines and unifies a number of concepts while preserving great generality. In addition, the mentioned equivalence shows for the first time how to extend the theory of desirability to imprecise non-linear utility, thus enabling us to formulate one of the most powerful self-consistent theories of reasoning and decision-making available today.
\end{abstract}

\section{Introduction}

Our main goal in this paper is to establish a bridge between the axiomatisations of incomplete preferences and imprecise probability models.

\subsection{Two Theories}

Von Neumann and Morgestern (1947), Anscombe and Aumann (1963) and Savage (1954) have shown long ago that a rational decision maker can be regarded as taking decisions by maximising his expected utility. This view has had an enormous impact in various fields of research. In artificial intelligence, in particular, it is at the basis of all Bayesian inference 
models as well as in any process of decision making, for instance in robotics, planning, classification, to name just a few.

Yet, many authors, including von Neumann, Morgenstern, Anscombe and Aumann themselves, have soon recognised that it is not realistic to assume that the decision maker can always compare alternatives; in some cases he may just not know which one to prefereven if only because he lacks information about them. In this case we talk of incomplete, or partial, preferences (for some applications in artificial intelligence see, e.g., Corani \& Zaffalon, 2008, Kikuti et al., 2011, de Bock \& de Cooman, 2014).

Axiomatisations of rational decision making with incomplete preferences came much later, though, through the works of Seidenfeld et al. (1995), Bewley (2002), Nau (2006), and, more recently, Ok et al. (2012) and Galaabaatar and Karni (2013). ${ }^{1}$ These works build upon the analytical framework of Anscombe and Aumann so as to represent rational (or coherent) preferences through sets of expected utilities; the disagreeing decisions these may lead to account for the incomplete nature of preferences.

In parallel, other researchers were developing a theory of imprecise probability (sets of probabilities). It started from de Finetti's view of expectation as a primitive concept: that of a fair price for a gamble (a bounded variable); de Finetti (1937) then deduced probability by imposing a single axiom of self-consistency on given expectations. Smith (1961) suggested that de Finetti's approach could be extended to account for the case that probabilities are indeterminate or not precisely specified. Williams (1975) made Smith's ideas precise, by giving an axiomatisation that is again based on a notion of self-consistency, which is called coherence since then. Walley (1991) popularised this notion through his theory of imprecise probability, which is largely based on Williams' (see Augustin et al., 2014, for a collection of introductory papers on imprecise probability and some main applications to artificial intelligence).

At the basis of the theories of both Williams (1975) and Walley (1991), there is the notion of a set of desirable gambles. ${ }^{2}$ The set is said to be coherent if it satisfies some axioms of rationality. Lower and upper expectations are derived from the coherent set of gambles, and are shown to be equivalent to sets of probabilities. The important point is, however, that coherent sets of desirable gambles can be conditioned, marginalised, extended to bigger spaces, and so on, without ever needing to talk about (sets of) probabilities. This is remarkable since coherent sets of desirable gambles can deal with non-Archimedean problems. Unlike for standard probability models, but similarly to lexicographic probabilities and full conditional measures, we can for instance condition a coherent set of gambles on an event of probability zero and obtain a well defined, informative model.

\subsection{Equivalence}

At the core of both the theory of desirability and of incomplete preferences is the geometrical structure of convex cones. This is well known since the start of either theory (Aumann, 1962; Smith, 1961). Moreover, it is well known that desirability and preference are tightly related

1. See the work of Dubois et al. (2003) for the related problem of axiomatising qualitative preferences.

2. Independently of Williams' and Walley's work, Seidenfeld et al. (1990) call them favourable gambles; in finance, Artzner et al. (1999) call them acceptable risks. 
concepts. So it is quite surprising that the two theories have not explicitly been related so far.

Part of the reason could be that the theories are based on different objects: gambles (desirability) and horse lotteries, or acts (incomplete preferences). But actually these two objects become very similar once we allow for vector-valued gambles: ${ }^{3}$ by doing so, in this paper we provide a simple proof of the general equivalence between desirability and partial preferences under one, widely used, requirement about the latter - that there is an act worse than all the others. Interestingly, the equivalence holds also for the corresponding notions of Archimedeanity (which allows us to represent preferences, or our desirable gambles, using probabilities and utilities).

\subsection{Main Contributions}

The mentioned equivalence has a major implication: it shows that by using vector-valued gambles, we can immediately extend desirability to deal with imprecise non-linear utility. What we get is arguably one of the most general theories of uncertainty and decision making available today; it is proposed here for the first time. To better appreciate such a result, consider that Walley (1991) regarded "a general theory of decision making, when both probabilities and utilities are imprecise" (pp. x-xi) as one of the main goals of future work.

Another important implication is that by mapping incomplete preferences into desirable gambles, we are allowed to formally regard decision problems as problems of uncertainty alone, where values (utilities) are mathematically represented as beliefs (probabilities) too; it is only the interpretation that differs. ${ }^{4}$ This makes the whole desirability toolset available for use in decision making in the general context of incomplete preferences.

Probably the brightest outcome of this implication in the paper is the proof that the decision-theoretic concept of state independence - the assumption that a decision maker holds separate models for beliefs and values - is nothing but strong independence in imprecise probability: that is, the widely used idea of expressing independence using a set of stochastically independent models. Besides making the assumption of state independence very clear, this also makes its limitations obvious, and in fact it leads us to propose a weaker, and arguably better notion. Moreover, and conversely, by exploiting the traditional decision-theoretic axiomatisation of state independence, we obtain the first behavioural interpretation of strong independence: this notion has in fact been widely regarded so far, and to some extent criticised, only as a product of the sensitivity analysis interpretation of imprecise probability. Cozman (2012, sec. 5) makes a further step by arguing that strong independence "stays uncomfortably between the more intuitive concept of epistemic independence and the easier to handle concept of complete independence", which, as we understand it, means that strong independence is neither based on actual sensitivity analysis nor it has a behavioural interpretation. Here we eventually find a comfortable place for strong independence within the behavioural interpretation.

Along similar lines, we reconsider the decision-theoretic notions of preferences that express complete beliefs and incomplete values and the opposite condition. These two cases

3. In fact, $\mathrm{Nau}(2008,2016)$ has allowed for them and, even though he has not made the link with desirability, has actually derived the axioms of desirability in a special case. See Section 6.2 for details.

4. Drèze (1971) has already made this observation, as, more recently, has Nau (2008). 
are treated by different axioms in the literature. Thanks to desirability, we show here that they are subject to the very same, and simple, formulation.

The question of Archimedeanity deserves special mention as well. We observe, apparently for the first time, that the classical decision-theoretic notion of Archimedeanity is incompatible with a vacuous state of preference. This motivates us to propose a weaker notion that exhibits better properties. Key to this result, as well as to the overall equivalence between preference and desirability, is that the preference relation admits a worst act. We show that the existence of the worst act can essentially be assumed without loss of generality. We go on to give a quite detailed analysis of different notions of Archimedeanity and their relations.

All these results have two dimensions of generality that are worth remarking. The first is that they are obtained directly at the level of the cone of desirable gambles: for this reason, they apply smoothly both to Archimedean and non-Archimedean problems, thus covering the broadest range of problems. This should be contrasted with the traditional decision-theoretic developments, which are for the most part obtained in the special case where preferences are expressed via probabilities and utilities. The second is that we do not place restrictions on the cardinality of the space of possibility, which can then be (also uncountably) infinite.

\subsection{Outline of the Paper}

To make the paper self-contained, we provide an introduction to desirability and preferences in Section 2. Section 3 details the equivalence of the two theories and discusses the extension of a preference relation to the worst act. Section 4 reconsiders the main result discussing some of its implications. New notions of state independence and completeness are proposed in Section 5.1 at the level of cones and specialised in Section 5.2 to sets of expected utilities. Section 5.3 proves the equivalence of the traditional formulation of state independence and strong independence. In Section 6 we compare our work with that of Nau (2006, 2008, 2016), Galaabaatar and Karni (2013) and Seidenfeld et al. (1995). Discussion and conclusions are gathered in Section 7. Appendix A deepens the study of different notions of Archimedeanity. Appendix B contains the proofs of all the results in the paper. Finally, Appendix C includes a list of the symbols used throughout the paper, and a graphical view of the relations between some main concepts.

\section{Preliminary Notions}

In this Section, we introduce the main features of sets of desirable gambles and preference relations that shall be needed in the remainder of the paper.

\subsection{Desirable Gambles}

We provide a very quick introduction to desirability in Section 2.1.1 for the reader that prefers to skip details, whereas a detailed one is given in Section 2.1.2. 


\subsubsection{In SHORT}

We consider a possibly infinite set of states $\Omega$. We use $\mathcal{L}(\Omega)$ to denote the set of all gambles on $\Omega$, i.e., bounded mappings from $\Omega$ to real-valued amounts of linear utility. $\mathcal{R}$ normally denotes a subset of $\mathcal{L}(\Omega)$ consisting of 'desirable' gambles having the property that they are coherent, which means that they satisfy some rationality axioms (D1-D4 below). A coherent set of desirable gambles in turn determines a so-called coherent lower prevision $\underline{P}$ on $\mathcal{L}(\Omega)$ (which has the interpretation of a lower expectation). In turn, $\underline{P}$ determines a set of linear previsions $P$, denoted by $\mathcal{M}(\underline{P})$, which can be uniquely associated with a set of finitely additive probabilities. ${ }^{5}$ Coherent sets of desirable gambles, as well as coherent lower previsions and sets of probabilities, can be conditioned, marginalised or extended. The former are however more general than the latter, in that they can readily deal with conditioning on events of probability zero or with choices under zero expectation, for which the traditional, Kolmogorovian, approach to probability is not applicable.

\subsubsection{In Detail}

We next introduce in more detail the basic theory of sets of desirable gambles; for a deeper account of the results and concepts mentioned in this section, we refer to the work of Walley (1991, sec. 3.7), Couso and Moral (2011), de Cooman and Miranda (2012), de Cooman and Quaeghebeur (2012), Miranda (2008), Miranda and Zaffalon (2010) and Augustin et al. (2014, ch. 1).

Let $\Omega$ denote the set of possible outcomes of an experiment, that is, the space of possibilities. We let the cardinality of $\Omega$ be general, so $\Omega$ can be infinite. A gamble $f: \Omega \rightarrow \mathbb{R}$ is a bounded real-valued function of $\Omega$. It is interpreted as an uncertain reward in a linear utility scale. Accepting a gamble $f$ is regarded as a commitment to receive $f(\omega)$ whatever $\omega$ occurs.

We take a set of desirable gambles as a primitive model of uncertainty about $\Omega$.

Denote by $\mathcal{L}(\Omega)$ the set of all the gambles on $\Omega$ and by $\mathcal{L}^{+}(\Omega):=\{f \in \mathcal{L}(\Omega): f \geqslant 0\}$ the subset of the positive gambles, where $f \geq 0$ means that $f \geq 0$ and $f \neq 0$. We denote these sets also by $\mathcal{L}$ and $\mathcal{L}^{+}$, respectively, when there can be no ambiguity about the space involved.

Definition 1 (Coherent set of gambles). $\mathcal{R}$ is said to be a coherent set of desirable gambles in $\mathcal{L}$ if and only if it satisfies the following conditions:

D1. $\mathcal{L}^{+} \subseteq \mathcal{R}$ [Accepting Partial Gains];

D2. $0 \notin \mathcal{R}$ [Avoiding Null Gain];

D3. $f \in \mathcal{R}, \lambda>0 \Rightarrow \lambda f \in \mathcal{R}$ [Positive Homogeneity];

D4. $f, g \in \mathcal{R} \Rightarrow f+g \in \mathcal{R}$ [Additivity].

A coherent set of desirable gambles is thus a convex cone (D3, D4) that excludes the origin (D2) and that contains the positive gambles (D1).

5. Such a correspondence follows from a separating hyperplane theorem. 
Consider an event $B \subseteq \Omega$. We denote by $B$ both the subset of $\Omega$ and its indicator function $I_{B}$ (that equals one in $B$ and zero elsewhere). Using this convention, we can multiply $B$ and a gamble $f$ obtaining a gamble $B f$ given for all $\omega \in \Omega$ by

$$
B f(\omega)= \begin{cases}f(\omega) & \text { if } \omega \in B \\ 0 & \text { otherwise }\end{cases}
$$

Definition 2 (Conditioning for gambles). Consider a coherent set of desirable gambles $\mathcal{R}$ on $\mathcal{L}$ and let $B$ be a non-empty subset of $\Omega$. The set of desirable gambles conditional on $B$ derived from $\mathcal{R}$ is defined as

$$
\mathcal{R} \mid B:=\{f \in \mathcal{R}: f=B f\} .
$$

There is a natural correspondence between $B f$ and the restriction of $f$ to $B$, whence we can also put $\mathcal{R} \mid B$ in relation with

$$
\mathcal{R}\rfloor B:=\left\{f_{B} \in \mathcal{L}(B):(\exists f \in \mathcal{R} \mid B)(\forall \omega \in B) f(\omega)=f_{B}(\omega)\right\},
$$

which is a coherent set of desirable gambles in $\mathcal{L}(B) . \mathcal{R} \mid B$ and $\mathcal{R}\rfloor B$ are equivalent representations of the conditional set.

Definition 3 (Marginalisation for gambles). Let $\mathcal{R}$ be a coherent set of desirable gambles in the product space $\Omega \times \Omega^{\prime}$, where $\Omega, \Omega^{\prime}$ are two logically independent sets. The $\Omega$ marginal set of desirable gambles on $\mathcal{L}\left(\Omega \times \Omega^{\prime}\right)$ induced by $\mathcal{R}$ is defined as

$$
\mathcal{R}_{\Omega}:=\left\{f \in \mathcal{R}:(\forall \omega \in \Omega)\left(\forall \omega_{1}^{\prime}, \omega_{2}^{\prime} \in \Omega^{\prime}\right) f\left(\omega, \omega_{1}^{\prime}\right)=f\left(\omega, \omega_{2}^{\prime}\right)\right\} .
$$

Since $\mathcal{R}_{\Omega}$ is made up of gambles that depend on $\Omega$ only (we call them $\Omega$-measurable gambles), we can establish a bijection between $\mathcal{R}_{\Omega}$ and

$$
\mathcal{R}_{\Omega}^{\prime}:=\left\{g \in \mathcal{L}(\Omega):(\exists f \in \mathcal{R})(\forall \omega \in \Omega)\left(\forall \omega^{\prime} \in \Omega^{\prime}\right) g(\omega)=f\left(\omega, \omega^{\prime}\right)\right\} .
$$

$\mathcal{R}_{\Omega}^{\prime}$ is a coherent set of gambles in $\mathcal{L}(\Omega)$.

A coherent set of desirable gambles encompasses a probabilistic model for $\Omega$ made of lower and upper expectations (also called previsions after de Finetti):

Definition 4 (Coherent lower and upper previsions). Let $\mathcal{R}$ be a coherent set of desirable gambles in $\mathcal{L}$. For all $f \in \mathcal{L}$, let

$$
\underline{P}(f):=\sup \{\mu \in \mathbb{R}: f-\mu \in \mathcal{R}\}
$$

it is called the lower prevision of $f$. The conjugate value given by $\bar{P}(f):=-\underline{P}(-f)$ is called the upper prevision of $f$. The functionals $\underline{P}, \bar{P}: \mathcal{L} \rightarrow \mathbb{R}$ are respectively called a coherent lower prevision and a coherent upper prevision.

Definition 5 (Linear prevision). Let $\underline{P}, \bar{P}$ be coherent lower and upper previsions on $\mathcal{L}$. If $\underline{P}(f)=\bar{P}(f)$ for some $f \in \mathcal{L}$, then we call the common value the prevision of $f$ and we denote it by $P(f)$. If this happens for all $f \in \mathcal{L}$ then we call the functional $P$ a linear prevision. 
A coherent lower prevision $\underline{P}$ has a set of dominating linear previsions:

$$
\mathcal{M}(\underline{P}):=\{P \text { linear prevision }:(\forall f \in \mathcal{L}) P(f) \geq \underline{P}(f)\},
$$

which turns out to be closed ${ }^{6}$ and convex. Since each linear prevision is in a one-to-one correspondence with a finitely additive probability, we can regard $\mathcal{M}(\underline{P})$ also as a set of probabilities (or a credal set). Moreover, $\underline{P}$ is the lower envelope of the previsions in $\mathcal{M}(\underline{P})$ :

$$
(\forall f \in \mathcal{L}) \underline{P}(f)=\min \{P(f): P \in \mathcal{M}(\underline{P})\} .
$$

The coherent upper prevision $\bar{P}$ is the upper envelope of the same set; as a consequence, it follows that $\underline{P}(f) \leq \bar{P}(f)$ for all $f \in \mathcal{L}$.

Definition 6 (Strict desirability). A coherent set of gambles $\mathcal{R}$ is said to be strictly desirable if it satisfies the following condition:

D0. $f \in \mathcal{R} \backslash \mathcal{L}^{+} \Rightarrow(\exists \delta>0) f-\delta \in \mathcal{R}$ [Openness],

where addition of a gamble with a constant is meant pointwise.

Strict desirability means that $\mathcal{R} \backslash \mathcal{L}^{+}$does not include its topological border. By an abuse of terminology, $\mathcal{R}$ is said to be open too. ${ }^{7}$

There is a one-to-one correspondence between coherent lower previsions and strictly desirable sets: from $\underline{P}$ we can induce set

$$
\mathcal{R}:=\{f \in \mathcal{L}: f \gtrless 0 \text { or } \underline{P}(f)>0\} ;
$$

$\mathcal{R}$ is coherent and strictly desirable and moreover induces $\underline{P}$ through Eq. (5).

Remark 1 (General vs strict and almost desirability). Note that a general coherent set of desirable gambles is a cone that need not be open nor closed. The closure of a coherent set of desirable gambles is called a set of almost-desirable gambles by Walley (1991, sec. 3.7.3). Strict desirability, as we have seen, is associated with open cones. Both almost and strictly desirable gambles are in a one-to-one correspondence with, and are therefore as informative as, a closed convex set of linear previsions. The extra generality of sets of desirable gambles makes them useful when dealing with the problem of conditioning on sets of probability zero, or of choosing between two options under zero expectation (Zaffalon $\mathcal{G}_{3}$ Miranda, 2013, sec. 5; see also Dubins, 1975; de Bock \&6 de Cooman, 2015a; Coletti E6 Scozzafava, 2002 for other approaches to this problem). It is this extra expressiveness that allows us to deal, later on in the paper, with decision problems in great generality. (For axiomatisations of incomplete preferences involving lower previsions, we refer to the works discussed in Section 6. Other works, more weakly related to ours, are Gilboa \&5 Schmeidler, 1989; Gilboa et al., 2010.)

6. In the weak* topology, which is the smallest topology for which all the evaluation functionals given by $f(P):=P(f)$, where $f \in \mathcal{L}$, are continuous.

7. A note of caution to prevent confusion in the reader: the adjective 'strict' denotes two unrelated things in desirability and in preferences. In preferences it characterises irreflexive relations, while in desirability it formalises an Archimedean condition as it will become clear in Section 3. We are keeping the same adjective in both cases for historical reasons and given that there should be no possibility to create ambiguity by doing so. 
Note also that with coherent sets of desirable gambles we need not enter the controversy as to whether or not we should use the 'regularity assumption', which prescribes that probabilities of possible events should be positive (see Pedersen, 2014). Such an assumption goes back to an important article by Shimony (1955). In the language of this paper, Shimony argued that de Finetti's framework could lead to the questionable undesirability of a positive gamble in case zero probabilities were present, given that the prevision (expectation) of such a gamble could be zero. In other words, Shimony argued, as we do, that D1 is an axiom of rationality. This led Shimony and a number of later authors, among whom Carnap and Skyrms, to advocate strengthening de Finetti's theory by requiring regularity. But it has originated also much controversy given the very constraining nature of regularity on probabilistic models. Between requiring regularity and dropping the desirability of positive gambles, coherent sets of desirable gambles actually offer a third possible way that keeps all desiderata together: events can have zero probability, positive gambles are desirable, and all operations, such as conditioning or comparisons, are well defined and informative. $\diamond$

Definition 7 (Conditional coherent lower and upper previsions). Let $\mathcal{R}$ be a coherent set of desirable gambles in $\mathcal{L}(\Omega)$ and $B$ a non-empty subset of $\Omega$. For all $f \in \mathcal{L}(\Omega)$, let

$$
\underline{P}(f \mid B):=\sup \{\mu \in \mathbb{R}: B(f-\mu) \in \mathcal{R}\}
$$

be the conditional lower prevision of $f$ given $B$. The conjugate value given by $\bar{P}(f \mid B):=$ $-\underline{P}(-f \mid B)$ is called the conditional upper prevision of $f$. The functionals $\underline{P}(\cdot \mid B), \bar{P}(\cdot \mid B)$ : $\mathcal{L}(\Omega) \rightarrow \mathbb{R}$ are respectively called a conditional coherent lower prevision and a conditional coherent upper prevision.

Denote by $\inf _{B} f$ the infimum value that $f$ takes on $B . \underline{P}(\cdot \mid B)$ satisfies the following conditions for all $f \in \mathcal{L}(\Omega)$ and all real $\lambda>0$ :

CC1. $\underline{P}(f \mid B) \geq \inf _{B} f$

CC2. $\underline{P}(\lambda f \mid B)=\lambda \underline{P}(f \mid B)$;

CC3. $\underline{P}(f+g \mid B) \geq \underline{P}(f \mid B)+\underline{P}(g \mid B)$.

These conditions are sometimes used as axioms of coherent conditional lower previsions, when not taking desirability as the primitive concept. Note that coherent lower previsions can be defined as a special case of conditional ones obtained when $B=\Omega$.

Definition 8 (Maximal coherent set of gambles). Let $\mathcal{R}$ be a coherent set of desirable gambles. It is called maximal if

$$
(\forall f \in \mathcal{L} \backslash\{0\}) f \notin \mathcal{R} \Rightarrow-f \in \mathcal{R} .
$$

Requiring maximality is tantamount to assuming complete preferences.

Finally, it is important for this paper to also say something about conglomerability, which is a condition that depends on the choice of a partition $\mathcal{B}$ of $\Omega:^{8}$

8. Note that we should call it $\mathcal{B}$-conglomerability, but we drop $\mathcal{B}$ given that in this paper we shall always consider the same partition. 
D5. $(\forall B \in \mathcal{B}) B f \in \mathcal{R} \cup\{0\} \Rightarrow f \in \mathcal{R} \cup\{0\}$ [Conglomerability].

D5 follows from $\mathrm{D} 4$ when $\mathcal{B}$ is finite. The rationale behind $\mathrm{D} 5$ is that if $f$ is desirable conditional on $B$, and this holds for all $B \in \mathcal{B}$, then it should also be unconditionally desirable. We refer to the work by Miranda et al. (2012) and Walley (1991, ch. 6) for a discussion of conglomerability in terms of sets of desirable gambles.

Definition 9 (Marginal extension for gambles). Let $\mathcal{R}_{\Omega}$ be a marginal coherent set of gambles in $\mathcal{L}\left(\Omega \times \Omega^{\prime}\right)$ and $\mathcal{R} \mid\{\omega\}$, for all $\omega \in \Omega$, be conditional coherent sets. Let

$$
\mathcal{R} \mid \Omega:=\left\{h \in \mathcal{L}\left(\Omega \times \Omega^{\prime}\right):(\forall \omega \in \Omega) h(\omega, \cdot) \in \mathcal{R} \mid\{\omega\} \cup\{0\}\right\} \backslash\{0\}
$$

be a set that conglomerates all the conditional information along the partition $\left\{\{\omega\} \times \Omega^{\prime}\right.$ : $\omega \in \Omega\}$ of $\Omega \times \Omega^{\prime}$ (by an abuse of notation, we denote this partition by $\Omega$ too). Then the following coherent and $\Omega$-conglomerable set of gambles:

$$
\hat{\mathcal{R}}:=\left\{g+h: g \in \mathcal{R}_{\Omega} \cup\{0\}, h \in \mathcal{R} \mid \Omega \cup\{0\}\right\} \backslash\{0\},
$$

is called the marginal extension of the given marginal and conditional information.

The marginal extension is a generalisation of the law of total expectation to desirable gambles. It can be defined for lower previsions too (see for instance Walley, 1991, sec. 6.7 and also Miranda \& de Cooman, 2007). To this end, we first give some preliminary notions.

Definition 10 (Separately coherent conditional lower prevision). Let $\mathcal{B}$ be a partition of $\Omega$ and $\underline{P}(\cdot \mid B)$ a coherent lower prevision conditional on $B$ for all $B \in \mathcal{B}$. Then we call

$$
\underline{P}(\cdot \mid \mathcal{B}):=\sum_{B \in \mathcal{B}} B \underline{P}(\cdot \mid B)
$$

$a$ separately coherent conditional lower prevision.

For every gamble $f, \underline{P}(f \mid \mathcal{B})$ is the gamble on $\Omega$ that equals $\underline{P}(f \mid B)$ for $\omega \in B$; so it is a $\mathcal{B}$-measurable gamble.

Definition 11 (Marginal coherent lower prevision). Let $\underline{P}$ be a coherent lower prevision on $\mathcal{L}\left(\Omega \times \Omega^{\prime}\right)$. Then the $\Omega$-marginal coherent lower prevision it induces is given by

$$
\underline{P}_{\Omega}(f):=\underline{P}(f)
$$

for all $f \in \mathcal{L}\left(\Omega \times \Omega^{\prime}\right)$ that are $\Omega$-measurable.

The $\Omega$-marginal is simply the restriction of $\underline{P}$ to the subset of gambles in $\mathcal{L}\left(\Omega \times \Omega^{\prime}\right)$ that only depend on elements of $\Omega$. For this reason, and analogously to the case of desirability, we can represent the $\Omega$-marginal in an equivalent way also through the corresponding lower prevision $\underline{P}_{\Omega}^{\prime}$ defined on $\mathcal{L}(\Omega)$.

Definition 12 (Marginal extension for lower previsions). Consider the possibility space $\Omega \times \Omega^{\prime}$ and its partition $\left\{\{\omega\} \times \Omega^{\prime}: \omega \in \Omega\right\}$. We shall denote this partition by $\Omega$ and its elements by $\{\omega\}$, with an abuse of notation. Let $\underline{P}_{\Omega}^{\prime}$ be a marginal coherent lower prevision and let $\underline{P}(\cdot \mid \Omega)$ be a separately coherent conditional lower prevision on $\mathcal{L}\left(\Omega \times \Omega^{\prime}\right)$. Then the marginal extension of $\underline{P}_{\Omega}^{\prime}$ and $\underline{P}(\cdot \mid \Omega)$ is the lower prevision $\underline{P}$ given for all $f \in \mathcal{L}\left(\Omega \times \Omega^{\prime}\right)$ by

$$
\underline{P}(f):=\underline{P}_{\Omega}^{\prime}(\underline{P}(f \mid \Omega)) .
$$




\subsection{Preference Relations}

We turn now our attention to the second pillar of this paper: preference relations.

\subsubsection{IN SHORT}

We shall consider $\mathcal{H}$ as the set of horse lotteries, i.e., all the mappings from the possibility space $\Omega$ to probability mass functions over $\mathcal{X}$, which is a set of prizes. They are interpreted as compound lotteries. The definition of a coherent preference relation on $\mathcal{H}$ is then introduced, namely one that satisfies some rationality axioms (A1, A2 below). Then $\mathcal{A}$ is introduced as the set of scaled differences of elements of $\mathcal{H}$ and $\mathcal{C}$ is introduced as the subset of $\mathcal{A}$ that equivalently represents the coherent preference relation.

\subsubsection{In Detail}

Let $\Omega$ denote, as before, the space of possibilities. In order to deal with preferences, we introduce now another set $\mathcal{X}$ of outcomes, or prizes. We take $\mathcal{X}$ to be a finite set. Moreover, we assume that all the pairs in $\Omega \times \mathcal{X}$ are possible or, which is equivalent, that $\Omega$ and $\mathcal{X}$ are logically independent.

The treatment of preferences relies on the basic notion of a horse lottery (Anscombe \& Aumann, 1963):

Definition 13 (Horse lottery). A horse lottery is a map $p: \Omega \times \mathcal{X} \rightarrow[0,1]$ such that $p(\omega, \cdot)$ is a probability mass function on $\mathcal{X}$ for all $\omega \in \Omega$.

Let us denote by $\mathcal{H}(\Omega \times \mathcal{X})$ the set of all horse lotteries on $\Omega \times \mathcal{X}$. Horse lotteries will also be called acts for short. In the following we shall use the notation $\mathcal{H}$ for the set of all the acts in case there is no possibility of ambiguity. An act $p$ for which $p\left(\omega_{1}, \cdot\right)=p\left(\omega_{2}, \cdot\right)$ for all $\omega_{1}, \omega_{2} \in \Omega$ is called a von Neumann-Morgenstern lottery; moreover, if such a $p(\omega, \cdot)$ is degenerate on an element $x \in \mathcal{X}$, then it is called a constant von Neumann-Morgenstern lottery and is denoted by the symbol $x$ : that is, $x(\omega, x)=1$ for all $\omega \in \Omega$.

A horse lottery $p$ is usually regarded as a pair of nested lotteries: at the outer level, the outcome $\omega \in \Omega$ of an experiment is employed to select the simple lottery $p(\omega, \cdot)$; this is used at the inner level to determine a prize $x \in \mathcal{X}$ (see Section 4.3 for more details). Horse lotteries can be related to a behavioural interpretation through a notion of preference.

Definition 14 (Coherent preference relation). A preference relation $\succ$ over horse lotteries is a subset of $\mathcal{H} \times \mathcal{H}$. It is said to be coherent if it satisfies the next two axioms:

A1. $(\forall p, q, r \in \mathcal{H}) p \nsucc p$ and $p \succ q \succ r \Rightarrow p \succ r$ [Strict Partial Order];

A2. $(\forall p, q \in \mathcal{H})(p \succ q \Leftrightarrow(\forall r \in \mathcal{H})(\forall \alpha \in(0,1]) \alpha p+(1-\alpha) r \succ \alpha q+(1-\alpha) r)$ [Mixture Independence].

If also the next axiom is satisfied, then we say that the coherent preference relation is Archimedean:

A3. $(\forall p, q, r \in \mathcal{H})(p \succ q \succ r \Rightarrow(\exists \alpha, \beta \in(0,1)) \alpha p+(1-\alpha) r \succ q \succ \beta p+(1-\beta) r)$ [Archimedeanity]. 
Let the set of scaled differences of horse lotteries be defined by

$$
\mathcal{A}(\mathcal{H}):=\{\lambda(p-q): p, q \in \mathcal{H}, \lambda>0\}
$$

We shall also denote this set simply by $\mathcal{A}$ in the following, if there is no possibility of confusion. The preference relation $\succ$ is characterised by the following subset of $\mathcal{A}$,

$$
\mathcal{C}:=\{\lambda(p-q): p, q \in \mathcal{H}, \lambda>0, p \succ q\}
$$

as the next proposition makes clear:

Proposition 1. Let $\succ$ be a coherent preference relation on $\mathcal{H} \times \mathcal{H}$. Then:

(i) For all $p, q \in \mathcal{H}$ and $\lambda>0$, it holds that $p \succ q \Leftrightarrow \lambda(p-q) \in \mathcal{C}$.

(ii) $\mathcal{C}$ is a convex cone that excludes the origin; it is empty if and only if $\succ$ is.

(iii) There is a one-to-one correspondence between coherent preference relations and convex cones in $\mathcal{A} \backslash\{0\}$.

(iv) Given $p, q, r, s \in \mathcal{H}$ such that $p-q=\lambda(r-s)$ for some $\lambda>0$, it holds that $p \succ q \Leftrightarrow$ $r \succ s$.

This equivalence between preferences and cones is not new, and dates back to the work of Aumann (1962). It has also been established, under various conditions, by Dubra et al. (2004, Lem. 2), Nau (2006, Thm. 1), and Galaabaatar and Karni (2013, Lem. 1), amongst others. The correspondence in (iii) needs suitable separation theorems in the traditional axiomatisation of preferences, as discussed in quite some detail by Nau $(2008,2016)$.

\section{Equivalence of Desirability and Preference}

In the generalisation of desirability we are about to describe in this section, $\mathcal{L}$ will be redefined to be the set of gambles on $\Omega \times \mathcal{X}$. These may be considered as vector-valued gambles whose elements are measured in 'probabilistic' units associated with the respective prizes (see Section 4.3 for more details). $\mathcal{R}$ henceforth will denote a coherent set of desirable gambles of this form, which is a subset of the new $\mathcal{L}$. Such a generalised definition of gambles makes them very close to horse lotteries. On this basis, the main result will be that the cone $\mathcal{C}$ that represents a coherent preference relation can be turned into a set of desirable gambles $\mathcal{R}$ and vice versa. Even more so, the equivalence will be shown to hold also for the notions of Archimedeanity and strict desirability. Key to these equivalences will be the notion of worst outcome, which will also lead us to a weakened definition of Archimedeanity. We shall also show that the existence of the worst outcome can be essentially assumed without loss of generality.

\subsection{Worst Outcome}

It is customary in the literature to assume that a coherent preference relation comes with a best and a worst act: 
Definition 15 (Best and worst acts). Let $\succ$ be a coherent preference relation and $p \in \mathcal{H}$. The relation has $a$ best act $b$ if $b \succ p$ for all $p \neq b$ and, similarly, it has $a$ worst act $w$ if $p \succ w$ for all $p \neq w$.

It follows from this definition that best and worst acts are necessarily unique. In this paper, however, we shall only be concerned with the worst act.

If we focus on constant von Neumann-Morgenstern lotteries, we see that a coherent preference relation $\succ$ also induces a strict partial order on the set of outcomes $\mathcal{X}$. Then a particular type of worst act would correspond to the case where this partial order also has a smallest element:

Definition 16 (Worst outcome). Let $\succ$ be a coherent preference relation. The relation has a worst outcome $z \in \mathcal{X}$ if $p \succ z$ for all $p \neq z .^{9}$

Now we wonder whether it is restrictive to assume that a coherent relation has a worst outcome compared to assuming that it has a worst act.

Lemma 2. Let $\succ$ be a coherent preference relation with worst act $w$. Then for each $\omega \in \Omega$, there is a (necessarily unique) $x_{\omega} \in \mathcal{X}$ such that $w\left(\omega, x_{\omega}\right)=1$.

In other words, a worst act has to be very similar to a worst outcome: a worst act tells us that for each $\omega \in \Omega$ there is always an element $x_{\omega} \in \mathcal{X}$ that is the worst possible; the difference is that such a worst element is not necessarily the same for all $\omega \in \Omega$. But at this point it is clear that we can reformulate the representation so as to work with the relation $\succ$ as if it had a worst outcome:

Proposition 3. Consider a coherent preference relation $\succ$ with a worst act $w$. Let $z$ be any element in $\mathcal{X}$. Define the bijection $\sigma: \mathcal{H} \rightarrow \mathcal{H}$ that, for all $\omega \in \Omega$, swaps the probabilities of $x_{\omega}$ and $z$ :

$$
\sigma(p)(\omega, x):= \begin{cases}p(\omega, z) & \text { if } x=x_{\omega} \\ p\left(\omega, x_{\omega}\right) & \text { if } x=z \\ p(\omega, x) & \text { otherwise }\end{cases}
$$

for all $p \in \mathcal{H}$. Application $\sigma$ induces a relation $\succ_{\sigma}$ by:

$$
p \succ_{\sigma} q \Leftrightarrow \sigma^{-1}(p) \succ \sigma^{-1}(q) .
$$

Then $\succ_{\sigma}$ is a coherent preference relation for which $z$ is the worst outcome. Moreover, the relation $\succ_{\sigma}$ is Archimedean if the relation $\succ$ is.

This means that if our original relation $\succ$ has a worst act $w$, we can always map it to a new relation, on the same product space $\Omega \times \mathcal{X}$, for which $z$ is the worst outcome and such that we can recover the original preferences from those of the new relation. So there is no loss of generality in assuming right from the start that $w=z$.

9. The ' $z$ ' symbol is a reminder for the gamble identically equal to zero, which the counterpart of act $z$ in the desirability formulation of preferences; see Section 3.2. 
In the rest of the paper, we shall indeed assume that a coherent preference relation has a worst outcome. However, as we shall show, this assumption is not without implications: the inclusion of a worst outcome is incompatible with the usual formulation of the Archimedean axiom, in the sense that it may entail the inclusion of preferences that were not present in the initial preference relation. This point will be clarified in Section 3.3. ${ }^{10}$

Remark 2 (Notation for the worst outcome). It is convenient to define some notation that is tailored for $z$. In particular, when we want to specify that the set of outcomes contains $z$, then we shall denote it by $\mathcal{X}_{z}$; otherwise we shall denote it by $\mathcal{X}$. In the latter case, it can either be that the set does not contain it or that the statements we make hold irrespectively of that. The distinction will be clear from the context. Note in particular that when the two sets are used together, the relation between them will always be that $\mathcal{X}_{z}=\mathcal{X} \cup\{z\}$. Moreover, we let $\mathcal{H}_{z}:=\mathcal{H}\left(\Omega, \mathcal{X}_{z}\right)$, besides the usual $\mathcal{H}:=\mathcal{H}(\Omega, \mathcal{X})$, and we use them accordingly. $\diamond$

Remark 3 (Assumption about the worst outcome). Note, in addition, that if $\mathcal{X}_{z}=$ $\{z\}$, then it is necessary that $\mathcal{H}_{z}=\{z\}$ and hence the only possible relation on $\mathcal{H}_{z} \times \mathcal{H}_{z}$ is the empty one. We skip this trivial case in the paper by assuming throughout that $\mathcal{X}_{z}$ contains at least two elements. $\diamond$

\subsection{Equivalence}

When $z$ exists, we can associate $\mathcal{C}$ with another set. Let us first define the operator that drops the $z$-components from an act:

Definition 17 (Projection operator). Consider a set of outcomes $\mathcal{X}_{z}$ that includes $z$. The projection operator is the linear map

$$
\psi: \mathcal{L}\left(\Omega \times \mathcal{X}_{z}\right) \rightarrow \mathcal{L}(\Omega \times \mathcal{X})
$$

defined by $\psi(h)(\omega, x):=h(\omega, x)$, for all $(\omega, x) \in \Omega \times \mathcal{X}$ and all $h \in \mathcal{L}\left(\Omega \times \mathcal{X}_{z}\right)$.

We shall use this operator to project horse lotteries in $\mathcal{H}_{z}$, or scaled differences of them, into gambles on $\Omega \times \mathcal{X}$.

It is then an easy step to show the following:

Theorem 4. Let $\succ$ be a preference relation on $\mathcal{H}_{z} \times \mathcal{H}_{z}$ and define

$$
\mathcal{R}:=\{\lambda \psi(p-q): p \succ q, \lambda>0\}=\psi(\mathcal{C}) .
$$

(i) If $\succ$ is coherent, then $\mathcal{R}$ is a coherent set of desirable gambles in $\mathcal{L}(\Omega \times \mathcal{X})$. Moreover, $p-q \in \mathcal{C} \Leftrightarrow \psi(p-q) \in \mathcal{R}$.

(ii) Conversely, any coherent set of desirable gambles $\mathcal{R}$ in $\mathcal{L}(\Omega \times \mathcal{X})$ defines a convex cone $\mathcal{C}$ that is equivalent to a coherent preference relation $\succ$ on $\mathcal{H}_{z} \times \mathcal{H}_{z}$ for which $z$ is the worst outcome.

10. The extension to a worst outcome 'without loss of generality' is also established by Seidenfeld et al. (1995), albeit in a framework with open sets of expectations. As we shall show in Section 6.3, their approach and results do not apply to closed sets. 
A similar correspondence between coherent preference relations and coherent sets of desirable gambles was established by Nau (2016, Thm. 3.3); see also some previous work by Nau (2008). One important difference with ours is that Nau works with weak preference relations $\succeq$, and as a consequence the associated set of desirable gambles $\overline{\mathcal{R}}$ can be shown to satisfy the axioms of almost-desirability from Walley (1991, sec. 3.7.3). A coherent set of almost-desirable gambles is the topological closure of a coherent set of desirable gambles, and turns out to be in a one-to-one correspondence with a set of linear previsions. It is less informative than a coherent set of desirable gambles, and it is also less well suited to conditioning, as discussed by Miranda and Zaffalon (2010, sec. 4) and de Cooman and Quaeghebeur (2012, sec. 3.1). ${ }^{11}$

Remark 4 (Vector-valued gambles). Note that $\mathcal{R}$ is made of gambles from $\mathcal{L}(\Omega \times$ $\mathcal{X})$, but only $\Omega$ is the space of possibilities. This marks a significant departure from the traditional definition of a set of desirable gambles, which would require in this case that $\Omega \times \mathcal{X}$, and not just $\Omega$, was the space of possibilities. Consider updating, for instance: in probability, we call updating the application of conditioning that is meant to capture how beliefs should change under the assumption that a certain event has occurred (conditioning is just the corresponding mathematical operation with no interpretation attached). In the current setting, it follows that updating is meaningful only when we condition on subsets of $\Omega$, as the elements of $\mathcal{X}$ simply do not 'occur' (and yet note that its application now updates both beliefs and values). In contrast, observe that conditioning, as a mere mathematical operation, is obviously legitimate also on subsets of $\mathcal{X}$. In line with these considerations, in general we interpret any $f \in \mathcal{R}$ as a vector-valued gamble: for each $\omega \in \Omega, f(\omega, \cdot)$ is the vector of associated rewards. In the following we shall omit the specification 'vector-valued' and refer more simply to the elements of $\mathcal{L}(\Omega \times \mathcal{X})$ as gambles. $\checkmark$

The projection operator gives us the opportunity to define a special type of preference:

Definition 18 (Objective preference). Given acts $p, q \in \mathcal{H}_{z}$ we say that $p$ is objectively preferred to $q$ if $\psi(p) \geq \psi(q)$ and $\psi(p) \neq \psi(q)$. We denote objective preference by $p \triangleright q$.

The idea is that $p$ is objectively preferred to $q$ because the probability $p$ assigns to outcomes, except for the outcome $z$, is always not smaller than that of $q$ while being strictly greater somewhere. An objective preference must indeed be included in any coherent preference relation: ${ }^{12}$

Proposition 5. Let $\succ$ be a coherent preference relation on $\mathcal{H}_{z} \times \mathcal{H}_{z}$ and $p, q \in \mathcal{H}_{z}$. Then $p \triangleright q \Rightarrow p \succ q$.

Objective preferences are therefore included in any coherent preference relation. If they are the only preferences in the relation, then we call the relation vacuous, as it is expressing only a non-informative type of preferences.

Perhaps surprisingly, the vacuity of a coherent preference relation turns out to be incompatible with the Archimedean axiom, except in trivial cases:

11. Another approach that is also more informative than almost-desirable gambles is that of Seidenfeld et al. (1995), who work with sets of expected utilities that need not be open nor closed. However, there are quite a few technical differences between our approach and theirs; we refer to Section 6.3 for a comparison.

12. In this paper we focus on pointwise comparisons of gambles; a more general approach would be that considered by de Cooman and Quaeghebeur (2012) and Quaeghebeur et al. (2015). 
Proposition 6. Let $\succ$ be the vacuous preference relation on $\mathcal{H}_{z} \times \mathcal{H}_{z}$, so that $p \succ q \Leftrightarrow p \triangleright q$. Then $\succ$ is Archimedean if and only if $|\Omega|=1$ and $\left|\mathcal{X}_{z}\right|=2$.

Nevertheless, it is possible to define a weaker version of the Archimedean axiom that does not lead to such an incompatibility and that is based on restricting the attention to nontrivial preferences:

Definition 19 (Weak Archimedean condition). Let $\succ$ be a coherent preference relation on $\mathcal{H}_{z} \times \mathcal{H}_{z}$. We say that the relation is weakly Archimedean if

wA3. $\left(\forall p, q \in \mathcal{H}_{z}\right) p \succ q$ and $p \not q \Rightarrow(\exists \alpha \in(0,1)) \alpha p+(1-\alpha) z \succ q$ [weak Archimedeanity].

In other words, wA3 retains the overall structure of the traditional condition A3, while focusing only on the non-trivial preferences. This is clarified in the following:

Proposition 7. Let $\succ$ be a coherent preference relation on $\mathcal{H}_{z} \times \mathcal{H}_{z}$. Consider the following condition:

$\mathrm{A}^{\prime} .\left(\forall p, q, r \in \mathcal{H}_{z}\right) p \succ q \succ r$ and $p \not q \Rightarrow(\exists \alpha \in(0,1)) \alpha p+(1-\alpha) r \succ q$.

Then $\succ$ satisfies wA3 if and only if it satisfies A3'. Moreover, the condition implies that

$$
\left(\forall p, q, r \in \mathcal{H}_{z}\right) p \succ q \succ r \text { and } q \not r \Rightarrow(\exists \beta \in(0,1)) q \succ \beta p+(1-\beta) r .
$$

Weak Archimedeanity and strict desirability are related as follows:

Theorem 8. (i) Let $\succ$ be a coherent preference relation on $\mathcal{H}_{z} \times \mathcal{H}_{z}$ satisfying wA3. Then the corresponding set $\mathcal{R}$ obtained through (13) is a coherent set of strictly desirable gambles.

(ii) Conversely, let $\mathcal{R}$ be a coherent set of strictly desirable gambles and let $\succ$ denote its associated coherent preference relation. Then $\succ$ is weakly Archimedean.

\subsection{Can We Add a Worst Outcome Without Loss of Generality?}

As Theorems 4 and 8 show, the presence of the worst outcome in a preference relation is the key to establishing the equivalence between preference and desirability. What if the worst outcome is not present?

Consider a coherent preference relation $\succ$ on $\mathcal{H} \times \mathcal{H}$. Let us investigate the problem of extending it in a minimal way to $\mathcal{H}_{z} \times \mathcal{H}_{z}$, where the new element $z \in \mathcal{X} \cup\{z\}=\mathcal{X}_{z}$ acts as the worst outcome. Let us start by making the notion of extension more precise:

Definition 20 (Extension to the worst outcome). Let $\succ$ be a coherent preference relation on $\mathcal{H} \times \mathcal{H}$. An extension of $\succ$ to the worst outcome is a coherent preference relation, denoted by the same symbol $\succ$, on $\mathcal{H}_{z} \times \mathcal{H}_{z}$ such that:

(i) $p \succ z$ for all $p \in \mathcal{H}_{z}, p \neq z$; 
(ii) $p^{\prime} \succ q^{\prime}$ whenever $p \succ q$ for some $p, q \in \mathcal{H}$, where $p^{\prime}, q^{\prime} \in \mathcal{H}_{z}$ are given by

$$
\begin{aligned}
p^{\prime}: \Omega \times \mathcal{X}_{z} & \rightarrow \mathbb{R} \\
(\omega, x) & \mapsto \begin{cases}p(\omega, x) & \text { if } x \neq z \\
1-\sum_{x \in \mathcal{X}} p(\omega, x) & \text { if } x=z,\end{cases}
\end{aligned}
$$

and similarly for $q^{\prime}$ with respect to $q$.

This definition is based on two truly minimal requirements. The first is that $z$ be the worst act. The second is that the original preferences be preserved by the extension. Note that we are not requiring that the extension to $\mathcal{H}_{z} \times \mathcal{H}_{z}$ does not include any new preferences in the original domain $\mathcal{H} \times \mathcal{H}$; indeed, as we shall show in Appendix A.2, this may be unavoidable when we want to preserve Archimedeanity.

On this basis, we have the following:

Theorem 9. Let $\succ$ be a coherent relation on $\mathcal{H} \times \mathcal{H}$ and let $\mathcal{C}$ be the corresponding cone. Then:

(i) A coherent preference relation $\succ$ on $\mathcal{H}_{z} \times \mathcal{H}_{z}$ extends the relation $\succ$ on $\mathcal{H} \times \mathcal{H}$ to the worst outcome if and only if for all $p, q \in \mathcal{H}_{z}$,

$$
p \succ q \text { whenever } p \triangleright q \text { or } \psi(p-q)=r-s \text { for some } r, s \in \mathcal{H} \text { such that } r \succ s \text {. }
$$

(ii) The smallest extension of $\succ$ to the worst outcome corresponds to the coherent set of desirable gambles given by:

$$
\mathcal{R}:=\{f \geq \lambda(r-s): r, s \in \mathcal{H}, r \succ s, \lambda>0\} \cup \mathcal{L}^{+} .
$$

(iii) Let $\underline{P}$ be the coherent lower prevision induced by $\mathcal{R}$. For all $f \in \mathcal{C}$, it holds that $\underline{P}(f)=0$. As a consequence, the minimal extension is weakly Archimedean if and only if $\mathcal{C}=\emptyset$.

Condition (14) has a simple interpretation. The part related to objective preferences has been discussed already after Definition 18: it means that objective preferences should indeed always be preferences. Concerning the remaining part, observe that $\psi(p-q) \in \mathcal{C}$ implies that $\sum_{x \neq z} p(\omega, x)=\sum_{x \neq z} q(\omega, x)$ for every $\omega \in \Omega$, and as a consequence $p(\omega, z)=q(\omega, z)$ for all $\omega \in \Omega$. This means that the evaluation of $p$ versus $q$ is not going to be based on their behaviour on $z$, since it is identical in the two cases; and for this reason one can determine the preference relation between $p$ and $q$ by relying only on the original relation (via $\mathcal{C}$ ).

The second part of Theorem 9 gives an important result: that there is always a leastcommittal extension of a coherent preference relation to the worst outcome. Saying that it is least-committal means that such an extension is obtained only using the coherence axioms A1, A2, and the assumption that $\mathcal{X}$ can be bounded by a worst outcome. ${ }^{13}$

On the other hand, in the third part of the theorem we obtain a result that is not as positive: that $\mathcal{R}$ is never a coherent set of strictly desirable gambles (except in the trivial

13. Under the assumption that the elements of $\mathcal{X}$ are prizes, that is achieved by simply letting $z$ represent the possibility that nothing is eventually won (from $\mathcal{X}$ ). 
case that $\mathcal{C}=\emptyset$ ). This means that the minimal extension cannot be reproduced by a set of linear previsions. Stated differently, we obtain that any coherent preference relation, when extended in a least-committal way to the worst outcome, is not going to be weakly Archimedean, as it follows from Theorem 8, and hence not Archimedean either, because of Proposition 7.

Still, one might wonder whether there are Archimedean extensions (these would introduce assessments other than those in (14)) so as to define the notion of minimal extension among the Archimedean ones. But this is actually not possible:

Corollary 10. Let $\succ$ be a coherent relation under the assumptions of Theorem 9 and $\mathcal{R}$ be as in (15). If there is a coherent set of strictly desirable gambles $\mathcal{R}_{1}$ that includes $\mathcal{R}$, then there is another coherent set of strictly desirable gambles $\mathcal{R}_{2}$ such that $\mathcal{R} \subsetneq \mathcal{R}_{2} \subsetneq \mathcal{R}_{1}$.

We can rephrase the corollary by saying that the notion of minimal extension among the weak Archimedean ones is ill-posed: in fact, the minimal extension should correspond to the intersection of all the Archimedean extensions; but this is just $\mathcal{R}$, the least-committal extension of Theorem 9.

\section{A Summary View and Some Discussion}

The analysis in the previous sections can be summarised by the following:

Theorem 11. There is a one-to-one correspondence between coherent preference relations on $\mathcal{H}_{z} \times \mathcal{H}_{z}$ for which $z$ is the worst outcome and coherent sets of desirable gambles on $\Omega \times \mathcal{X}$. Moreover the relation is weakly Archimedean if and only if the set of gambles is strictly desirable. In this case they give rise to the same representation in terms of sets of linear previsions.

In other words, from the mathematical point of view, it is completely equivalent to use coherent sets of desirable gambles on $\Omega \times \mathcal{X}$ in order to represent coherent preference relations on $\mathcal{H}_{z} \times \mathcal{H}_{z}$. That the two theories are essentially the same becomes even clearer if we consider that we can define a notion of preference directly on top of a coherent set of desirable gambles as shown by Walley (1991, sec. 3.7.7):

Definition 21 (Coherent preference relation for gambles). Given a coherent set of desirable gambles $\mathcal{R}$ in $\mathcal{L}(\Omega \times \mathcal{X})$ and gambles $f, g$, we say that $f$ is preferred to $g$ in $\mathcal{R}$ if $f-g \in \mathcal{R}$, and denote this by $f \succ g$.

It is trivial to show that the notion of preference we have just described is equivalent to the preference relation on horse lotteries that corresponds to $\mathcal{R}$ : this means that $f \succ g$ if and only if $p \succ q$ for all $p, q \in \mathcal{H}_{z}$ such that $p-q=\psi(\lambda(f-g))$ for some $\lambda>0$. This is also the reason why we use the same symbol in both cases.

All this suggests that we can think of establishing the foundations of rational decision making on gambles by evaluating the rationality of given assessments through D1-D4. Moreover, we can profit now from all the theory already developed for desirable gambles in order to study decision-theoretic problems in quite some generality. 


\subsection{Representing Utility (Values) by Uncertainty Models}

In this perspective, there is a feature of our proposed representation of decision-theoretic problems that is worth discussing even if only to make its usefulness explicit.

Let us consider the simplest setup to make things very clear: a finite $\Omega$ (as well as $\mathcal{X}$, as usual) and a coherent set of strictly desirable gambles $\mathcal{R}$ corresponding to the interior of a maximal set. This means that $\mathcal{R}$ is in a one-to-one correspondence with a joint mass function $\Pi$ on $\Omega \times \mathcal{X}$ that is the restriction of a linear prevision to the elements of the product space. $\Pi$ is a joint mass function in the sense that $\Pi(\omega, x) \geq 0$ for all $(\omega, x) \in \Omega \times \mathcal{X}$ and $\sum_{(\omega, x) \in \Omega \times \mathcal{X}} \Pi(\omega, x)=1$. Now, if we assume that $\Pi(\omega, x)>0$ for all $(\omega, x) \in \Omega \times \mathcal{X}$, then $\Pi$ becomes an equivalent representation of $\mathcal{R}$. Rewriting $\Pi$ by the law of total probability (marginal extension) we get:

$$
\Pi(\omega, x)=\Pi(\omega) \Pi(x \mid \omega)
$$

for all $(\omega, x) \in \Omega \times \mathcal{X}$. Here the implicit meaning of $\Pi(\omega)$ is that of the probability of $\omega$, whereas $\Pi(x \mid \omega)$ represents the utility of prize $x$ conditional on the occurrence of $\omega$.

The important point here is that in our approach the utility function is just another mass function, that is, one such that $\Pi(x \mid \omega) \geq 0, \sum_{x \in \mathcal{X}} \Pi(x \mid \omega)=1$ for all $\omega \in \Omega$. That is, the utility mass function is formally equivalent to a probability mass function, it is only our interpretation that differs. Interestingly, this very point was made long ago by Drèze (1971, p. 152); it was also taken up in recent years by Nau (2008). However, this observation does not seem to have been exploited to a very large extent so far. Our viewpoint is instead that it is very convenient to treat utility formally as probability, as we shall actually do in the rest of the paper.

An advantage of this representation is that we can directly exploit all the machinery and concepts already in place for probabilities also for utilities. For example, the notion of state independence trivially becomes probabilistic independence through (16), and this happens whenever for all $x \in \mathcal{X}$ it holds that

$$
\Pi\left(x \mid \omega^{\prime}\right)=\Pi\left(x \mid \omega^{\prime \prime}\right)
$$

for all $\omega^{\prime}, \omega^{\prime \prime} \in \Omega$, so that we are allowed to work with marginal mass functions for probability and utility. Instead, that state independence fails means that we have to work with the joint mass function $\Pi$.

Similar comments can also be made if we move back towards greater generality. For instance, if we relax the precision requirement of the previous example, what we get is a representation of a decision-theoretic problem by a closed convex set $\mathcal{M}$ of joint mass functions. State independence in this case will coincide with a notion of independence or irrelevance for credal sets. When state independence holds, preferences will be formed by combining a marginal credal set for probabilities $\mathcal{M}_{\Omega}$ with a marginal one for utilities $\mathcal{M}_{\mathcal{X}}$. Completeness of utilities will mean that $\mathcal{M}_{\mathcal{X}}$ contains only one mass function; vice versa for completeness of probabilities (all this will be detailed in Sections 5.1-5.3).

These credal sets are in a one-to-one correspondence with coherent lower previsions. This means that there will be a coherent lower prevision $\underline{P}(\cdot \mid\{\omega\})$ representing the lower envelope of the previsions that we can obtain from the credal set $\mathcal{M}_{\mathcal{X}}(\cdot \mid\{\omega\})$ of the utilities conditional on $\{\omega\}$; that is, by formally treating utilities as probabilities and computing the related expectations. Relaxing the example further, we can consider an infinite (not 
necessarily countable) space $\Omega$ and in this case the credal set $\mathcal{M}_{\Omega}$ will be made up of finitely additive probabilities.

What we find remarkable is also that we can easily obtain the quantities we need explicitly from sets of desirable gambles using established tools. In more traditional axiomatisations of preferences, separation theorems are usually aimed at obtaining a "proof of existence' of the linear functionals that represent probabilities and utilities, which then leads to a representation theorem. Our representation naturally appears to be more of a constructive nature, in the sense that we can use (8) to compute the elements of interest; for instance, we can obtain the lower utility mass of $x \in \mathcal{X}$ conditional on $\omega \in \Omega$ simply as

$$
\underline{P}\left(I_{\{x\}} \mid\{\omega\}\right)=\sup \left\{\mu \in \mathbb{R}: I_{\{\omega\}}\left(I_{\{x\}}-\mu\right) \in \mathcal{R}\right\} .
$$

The overall situation is very much alike when we finally move backwards to $\mathcal{R}$. This will be in general a 'joint' model for beliefs and values. When we decompose it into separate models, there will be a marginal set for beliefs $\mathcal{R}_{\Omega}$ and other models for values, like $\mathcal{R} \mid\{\omega\}$, which will be formally treated as usual as if they were models of conditional beliefs instead of values. And this will allow us to exploit all the tools and concepts already developed for desirability, like marginal extension, for instance.

Note that we are not claiming that all this is impossible to do in the traditional way of axiomatising preference. What we want to say is that our framework naturally represents decision-theoretic problems in which values (utilities) are formally treated as beliefs (probabilities); and that this helps to deal with these problems in a familiar way while exploiting well established tools for beliefs also for values.

\subsection{Weak Archimedeanity, Strict Preference and the Cardinality of Spaces}

The discussion in the previous section helps us also to uncover a question related to Condition A3 in relation to strict preference orderings. In fact, A3 is conceived to eventually lead to models that have to assign positive lower expected utility (prevision) to all the elements of the cone $\mathcal{C}$, or, equivalently, to all the gambles in $\mathcal{R}$. Remember that in the formulation based on $\mathcal{R}$, beliefs and values naturally turn out to be jointly represented through a set $\mathcal{M}$ of linear previsions, which are formally equivalent to a set of probabilities (in the sense that not only probability but also utility is mathematically represented through a 'probability' function). This enables us to see clearly that, under A3 and strict preference, it is necessary that $\Omega \times \mathcal{X}$ be countable: for $\mathcal{R}$ contains the positive gambles, among which that are the

indicator functions of the subsets of $\Omega \times \mathcal{X}$; and a probability can assign positive mass to countably many events at most.

Of course this is quite a limitation in the representation. This makes us appreciate that, by taking aside the positive gambles, the weak Archimedean condition wA3 is not subject to such a problem: it yields a representation through a set of linear previsions on gambles that can be defined on uncountable spaces.

\subsection{Is Utility Non-Linear After All?}

Throughout this paper we say that the presented setting allow us to deal with non-linear utility (or values). Here we would like to make such a claim precise, as it is subtler than it may seem at first. 
Suppose, to make things simple and concrete, that the prizes in $\mathcal{X}$ represent amounts of dollars. Moreover, assume, as in (16), that utility is represented by a single mass function $\Pi$, which we now take as state-independent: this means that for each $x \in \mathcal{X}, \Pi(x)$ is a number in $[0,1]$ that represents the utility of $x$ dollars, no matter the possible states $\omega \in \Omega$. Now, if we plotted dollars $(x \in \mathcal{X})$ vs. utilities $(\Pi(x))$, we could in principle see $\Pi$ take any shape. In this sense, utility is clearly non-linear in dollars, in general.

But do we ever apply utility directly to dollars in the framework of this paper? Actually not. To see why, consider a very simple experiment made of a single state $\omega$, that is, in which $|\Omega|=1$. This allows us to simplify the formalism by regarding gambles as functions of dollars alone, $f: \mathcal{X} \rightarrow \mathbb{R}$. Now suppose that $\mathcal{X}=\{1,1000\}$, i.e., that there are only two prizes: 1 dollar and 1000 dollars, and that there are gambles $f, g$ such that

$$
f(x)=\left\{\begin{array}{ll}
1000 & \text { if } x=1, \\
0 & \text { if } x=1000,
\end{array} \quad g(x)= \begin{cases}0 & \text { if } x=1 \\
1 & \text { if } x=1000\end{cases}\right.
$$

We wonder whether $f$ and $g$ are equivalent gambles. In order to be equivalent, it should hold that $1000 \Pi(1)=\Pi(1000)$; namely, that $\Pi$ is linear in dollars. Since this is just a special case, $f$ and $g$ are not equivalent in general. This means also that accepting $f$ will not get us 1000 times 1 dollar. But then what is the meaning of that 1000 in the definition of $f$ ?

Remember that we have derived gambles from horse lotteries by applying the projection operator; and by definition horse lotteries do not pay the prizes in $\mathcal{X}$ : they only return a probability over them. They essentially pay us in 'probability currency': these are probabilities that, properly scaled down, add up to our pre-existing probabilities to win the prizes to determine our new probability by which we could play a simple lottery that eventually returns the actual prizes. ${ }^{14}$ In the case of gambles, which - as opposed to horse lotteries - can take any real values, we can reformulate the same idea in an equivalent way by regarding them as returning lottery tickets. ${ }^{15}$ Gamble $f$, for instance, returns 1000 tickets that would lead us to get 1 dollar if the ticket eventually drawn at the simple lottery corresponded to one of those; gamble $g$ instead returns 1 lottery ticket corresponding to the possible prize of 1000 dollars.

Now, considered that the probability of winning a prize at a simple lottery is proportional to the number of corresponding lottery tickets one owns, we get that the utility is linear in the number of lottery tickets. In other words, under the 'probability currency' interpretation, we obtain that positive homogeneity (D3) and additivity (D4) must hold for gambles, or, equivalently, that mixture independence (A2) and transitivity (A1 deprived of irreflexivity) must hold in the case of horse lotteries.

What we get is that the achievement of a formalism capable of handling 'non-linear' utility in this paper, as discussed above (the non-linearity of $\Pi$ ), is actually obtained through linear assumptions (D3, D4). Such an apparent paradox is felt particularly strong when we

14. A subtle point is indeed that the probabilities returned by horse lotteries should not be interpreted as the probabilities used to play the simple lottery directly; otherwise, for instance, a probability 1 would mean that a prize is won for sure, which is not consistent with the assumption of linearity embodied by the mixture independence axiom (given that in general we have non-linear utility in the prizes).

15. The idea of a 'probability currency' appears to go back to Savage (1954) and Smith (1961). Walley (1991, sec. 2.2.2) has discussed in some detail how that idea leads gambles (restricted to the case of a single prize) to be interpreted as uncertain rewards in terms of lottery tickets that can be won or lost. 
confuse actual prizes with corresponding lottery tickets, since utility is non-linear in the actual prizes but linear in the lottery tickets. In particular, we should remember that while $\Pi$ is indeed the tool that allows us to compare different prizes in $\mathcal{X}$, it is targeted at eventually comparing gambles expressed in terms of lottery tickets: the conceptual step we should not take is to deduce that we can apply $\Pi$ directly to choose between gambles that return dollars instead of lottery tickets about dollars.

To summarise, our approach, in the tradition of Anscombe and Aumann (1963), models non-linearity in utility in the sense discussed above about $\Pi$. It does not model the case where gambles return actual prizes about which we may possibly have non-linear utility. That avenue is taken by Savage (1954), for instance: in fact, Savage's axiomatisation is often regarded as the prominent justification for the maximum expected utility approach when it comes to actual prizes. At the same time, it is the subject of substantial criticism, since maximising the expected utility of actual prizes does not seem to always yield sensible policies; this is notorously illustrated through the Allais paradox (1953).

\section{Decomposition and Completeness of Preferences}

Now we exploit the equivalence given in Section 3 to analyse the most important special cases that allow us to simplify a decision-theoretic problem through a decomposition of preferences in beliefs and perceived values (of consequences).

\subsection{In Terms of Sets of Desirable Gambles}

We begin by considering the case where our assessments are modelled by sets of desirable gambles.

\subsubsection{State Independence}

Consider the following:

Definition 22 ( $\Omega$-X $\mathcal{X}$ irrelevant product for gambles). A coherent set of gambles $\mathcal{R}$ on $\Omega \times \mathcal{X}$ is called an $\Omega-\mathcal{X}$ irrelevant product of its marginal sets of gambles $\mathcal{R}_{\Omega}^{\prime}, \mathcal{R}_{\mathcal{X}}^{\prime}$ if it includes the set

$$
\mathcal{R} \mid \Omega:=\left\{h \in \mathcal{L}(\Omega \times \mathcal{X}):(\forall \omega \in \Omega) h(\omega, \cdot) \in \mathcal{R}_{\mathcal{X}}^{\prime} \cup\{0\}\right\} \backslash\{0\} .
$$

These products have been considered, in another context and for lower previsions, by Miranda and Zaffalon (2015). The rationale of the definition is the following. First, the requirement that $h(\omega, \cdot) \in \mathcal{R}_{\mathcal{X}}^{\prime} \cup\{0\}$ is there so that the inferences conditional on $\{\omega\}$ encompassed by $\mathcal{R} \mid \Omega$ should yield the marginal set $\mathcal{R}_{\mathcal{X}}^{\prime}$. This is just a way to formally state that $\omega$ is irrelevant to $\mathcal{X}$ : that is, the assumption that getting to know $\omega$ would have no implication for $\mathcal{X}$. The same is repeated for every $\omega$, so that $\mathcal{R} \mid \Omega$ can be regarded as being born out of aggregating all the irrelevant conditional sets. Given that $\Omega$ is possibly infinite, this entails an assumption of conglomerability. ${ }^{16}$ Finally, that $\mathcal{R}$ contains $\mathcal{R} \mid \Omega$ is imposed to make sure that $\mathcal{R}$ is a model coherent with the irrelevance of beliefs on values.

16. Without such an assumption one could obtain uninformative preferences; see the work by Miranda and Zaffalon (2015, sec. 5.1) for details. 
If $\mathcal{R}$ satisfies Definition 22 we say it models state-independent preferences. The leastcommittal among these models is given by ${ }^{17}$

Proposition 12. Given two marginal coherent sets of desirable gambles $\mathcal{R}_{\Omega}, \mathcal{R}_{\mathcal{X}}$ defined on the subsets of $\mathcal{L}(\Omega \times \mathcal{X})$ given by the $\Omega$-measurable and $\mathcal{X}$-measurable gambles, respectively, their smallest $\Omega-\mathcal{X}$ irrelevant product is given by the marginal extension of $\mathcal{R}_{\Omega}$ and $\mathcal{R} \mid\{\omega\}:=$ $\mathcal{R}_{\mathcal{X}}$ for all $\omega \in \Omega$. Namely,

$$
\hat{\mathcal{R}}:=\left\{g+h: g \in \mathcal{R}_{\Omega} \cup\{0\}, h \in \mathcal{R} \mid \Omega \cup\{0\}\right\} \backslash\{0\} .
$$

Definition 22 and Eq. (18) represent our main proposal to model state-independent preferences. Note that it follows from Remark 4 that updating is meaningful only if applied to elements of $\Omega$, and therefore state independence should be an asymmetric notion (we should not consider updating on $\mathcal{X}$ ). In this sense, the present context of beliefs and values is one where the interest in an asymmetric notion of independence arises very naturally. For applications of irrelevance to the more traditional case of beliefs only, see for instance the work by de Cooman and Miranda (2009), de Bock and de Cooman (2014) and de Bock and de Cooman (2015b).

Having said this, we also note that symmetric notions are considered in the literature; whence we introduce a symmetrised version of Definition 22 so as to lay the ground for a connection with former proposals (that we shall deepen in Section 5.2.1 in the special case of lower previsions):

Definition 23 (Independent product for gambles). A coherent set of gambles $\mathcal{R}$ on $\Omega \times \mathcal{X}$ is called an independent product of its marginal sets of gambles $\mathcal{R}_{\Omega}, \mathcal{R}_{\mathcal{X}}$ if it includes $\mathcal{R} \mid \Omega$ and $\mathcal{R} \mid \mathcal{X}$, where

$$
\mathcal{R} \mid \mathcal{X}:=\left\{h \in \mathcal{L}(\Omega \times \mathcal{X}):(\forall x \in \mathcal{X}) h(\cdot, x) \in \mathcal{R}_{\Omega}^{\prime} \cup\{0\}\right\} \backslash\{0\} .
$$

These products were studied for finite spaces by de Cooman and Miranda (2012). Note that the above conditions hold in particular when $\mathcal{R}$ includes $\mathcal{R} \mid \Omega$ and

$$
\begin{aligned}
& (\forall \omega \in \Omega) \mathcal{R} \mid\{\omega\}=\mathcal{R}_{\mathcal{X}}, \\
& (\forall x \in \mathcal{X}) \mathcal{R} \mid\{x\}=\mathcal{R}_{\Omega} ;
\end{aligned}
$$

this is closer to the formulation by de Cooman and Miranda, and it is not required in general in the definition above.

An independent product models state-independent preferences since it includes the weakest $\Omega-\mathcal{X}$ irrelevant product. The smallest, or least-committal, independent product of two sets is given by the following proposition: ${ }^{18}$

Proposition 13 (Independent natural extension). Let $\mathcal{R}_{\Omega}^{\prime}, \mathcal{R}_{\mathcal{X}}^{\prime}$ be two marginal coherent sets of desirable gambles and let $\mathcal{R}|\Omega, \mathcal{R}| \mathcal{X}$ be given by Eqs. (17)-(19). Then the independent natural extension of $\mathcal{R}_{\Omega}^{\prime}, \mathcal{R}_{\mathcal{X}}^{\prime}$ is given by:

$$
\mathcal{R}_{\Omega}^{\prime} \otimes \mathcal{R}_{\mathcal{X}}^{\prime}:=\{g+h: g \in \mathcal{R}|\mathcal{X} \cup\{0\}, h \in \mathcal{R}| \Omega \cup\{0\}\} \backslash\{0\}
$$

17. We refer to the work by Moral (2005, sec. 2.4); de Cooman and Miranda (2012, Thm. 13) and de Bock and de Cooman (2015b, Thm. 8) for somewhat related results on finite referential spaces.

18. For related results when both $\Omega, \mathcal{X}$ are assumed to be finite, we refer to the work of de Cooman and Miranda (2012, Thm. 19) and de Bock and de Cooman (2015b, Prop. 13). 
It is the least-committal model for which beliefs on $\Omega$ are independent of values of $\mathcal{X}$, and that moreover satisfies conglomerability with respect to $\Omega$.

We need the independent natural extension in particular to be able to define a more informative independent product; our next definition is based on the work by de Cooman and Miranda (2012, sec. 8.2) for finite referential spaces.

Definition 24 (Strong product for gambles). The strong (independent) product of marginal coherent sets $\mathcal{R}_{\Omega}^{\prime}, \mathcal{R}_{\mathcal{X}}^{\prime}$ is defined as

$$
\mathcal{R}_{\Omega}^{\prime} \otimes \mathcal{R}_{\mathcal{X}}^{\prime}:=\cap\left\{\mathcal{D}_{\Omega} \otimes \mathcal{D}_{\mathcal{X}}: \mathcal{R}_{\Omega}^{\prime} \subseteq \mathcal{D}_{\Omega} \text { maximal and } \mathcal{R}_{\mathcal{X}}^{\prime} \subseteq \mathcal{D}_{\mathcal{X}} \text { maximal }\right\} .
$$

The strong product is the extension of a set of stochastically independent models to desirable gambles. It shall be studied in more detail in Section 5.2.

\subsubsection{State Dependence}

We say that a set of desirable gambles $\mathcal{R}$ models state-dependent preferences when it does not satisfy the conditions in Definition 22, i.e., when it does not include the set $\mathcal{R} \mid \Omega$ derived from its marginal $\mathcal{R}_{\mathcal{X}}$ and the assumption of $\Omega-\mathcal{X}$ irrelevance. This may arise for instance when the conditional sets of gambles $\mathcal{R} \mid\{\omega\}$ that $\mathcal{R}$ induces do not satisfy Eq. (20).

\subsubsection{Completeness}

So far we have discussed the general case of coherent sets of gambles $\mathcal{R}$ without discussing in particular the so-called complete or maximal coherent sets for which it holds that $f \notin$ $\mathcal{R} \Rightarrow-f \in \mathcal{R}$ for all $f \in \mathcal{L}, f \neq 0$. Given the equivalence between coherent sets of gambles in $\mathcal{L}(\Omega \times \mathcal{X})$ and coherent preferences in $\mathcal{H}_{z} \times \mathcal{H}_{z}$, discussing the case of maximal sets amounts to discussing the case of complete preferences.

Definition 25 (Completeness of beliefs and values for gambles). A coherent set of desirable gambles $\mathcal{R} \subseteq \mathcal{L}(\Omega \times \mathcal{X})$ is said to represent complete beliefs if $\mathcal{R}_{\Omega}$ is maximal. It is said to represent complete values if $\mathcal{R}_{\mathcal{X}}$ is maximal. Finally, if $\mathcal{R}$ is maximal, then it is said to represent complete preferences.

The rationale of this definition is straightforward: when we say, for instance, that $\mathcal{R}$ represents complete beliefs, we mean that there is never indecision between two options that are concerned only with $\Omega$. The situation is analogous in the case of complete values. Finally, in the case of complete preferences, the definition is just the direct application of the definition of maximality. If a coherent set of desirable gambles represents complete preferences, then it represents both complete beliefs and complete values, as shown by de Cooman and Miranda (2012, Prop. 22). The converse does not hold in general: we can construct a non-maximal coherent set of desirable gambles that represents both complete beliefs and values, and this also in the case of finite $\Omega$. We refer to de Cooman and Miranda (2012, app. A1) for an example.

\subsection{In Terms of Lower Previsions}

We now consider the case where preferences are modelled by means of a coherent lower prevision $\underline{P}$ on $\mathcal{L}(\Omega \times \mathcal{X})$. 


\subsubsection{State Independent Preferences}

Let us define the products we shall be concerned with.

Definition 26 ( $\Omega-\mathcal{X}$ irrelevant product for lower previsions). Given marginal coherent lower previsions $\underline{P}_{\Omega}^{\prime}, \underline{P}_{\mathcal{X}}^{\prime}$, let

$$
\underline{P}(f \mid\{\omega\}):=\underline{P}_{\mathcal{X}}^{\prime}(f(\omega, \cdot)),
$$

for all $f \in \mathcal{L}(\Omega \times \mathcal{X})$, and

$$
\underline{P}(\cdot \mid \Omega):=\sum_{\omega \in \Omega} I_{\{\omega\}} \underline{P}(\cdot \mid\{\omega\}) .
$$

Then a coherent lower prevision $\underline{P}$ on $\mathcal{L}(\Omega \times \mathcal{X})$ is called an $\Omega$-X irrelevant product of $\underline{P}_{\Omega}^{\prime}, \underline{P}_{\mathcal{X}}^{\prime}$ if

$$
\underline{P} \geq \underline{P}_{\Omega}^{\prime}(\underline{P}(\cdot \mid \Omega)) .
$$

In this case we also say that $\underline{P}$ models state-independent preferences.

Here $\underline{P}(\cdot \mid \Omega)$ plays the role that $\mathcal{R} \mid \Omega$ took in Section 5.1, that is, conglomerating all the conditional information. The concatenation $\underline{P}_{\Omega}^{\prime}(\underline{P}(\cdot \mid \Omega))$ is a marginal extension: in particular, it is the least-committal coherent $\Omega$-conglomerable model built out of the given marginals and the assessment of irrelevance that defines $\underline{P}(\cdot \mid \Omega)$ through $\underline{P}_{\mathcal{X}}^{\prime}$. Every coherent lower prevision that dominates $\underline{P}_{\Omega}^{\prime}(\underline{P}(\cdot \mid \Omega))$ is compatible with the irrelevance assessment but is also more informative than that:

Proposition 14. Given two marginal coherent lower previsions $\underline{P}_{\Omega}^{\prime}, \underline{P}_{\mathcal{X}}^{\prime}$, their smallest $\Omega-\mathcal{X}$ irrelevant product is given by the marginal extension

$$
\underline{P}_{\Omega}^{\prime}(\underline{P}(\cdot \mid \Omega)) .
$$

We refer to the work of Walley (1991, sec. 6.7), Miranda and de Cooman (2007), de Cooman and Miranda (2009) and de Cooman and Hermans (2008) for additional information on the marginal extension and its use in a number of different contexts.

As in the case of desirability, we can define more precise irrelevant products. Among them there are the independent products: ${ }^{19}$

Definition 27 (Independent product for lower previsions). Consider marginal coherent lower previsions $\underline{P}_{\Omega}^{\prime}, \underline{P}_{\mathcal{X}}^{\prime}$. We say that a coherent lower prevision $\underline{P}$ on $\mathcal{L}(\Omega \times \mathcal{X})$ is an independent product of $\underline{P}_{\Omega}^{\prime}, \underline{P}_{\mathcal{X}}^{\prime}$ if it is both an $\Omega-\mathcal{X}$ irrelevant product and an $\mathcal{X}-\Omega$ irrelevant product.

The weakest independent product is again called the independent natural extension (for more information, see de Cooman \& Miranda, 2012; Miranda \& Zaffalon, 2015). The, more informative, strong (independent) product is simply defined as a lower envelope of stochastic products:

19. This definition is equivalent to the one established by Miranda and Zaffalon (2015, Def. 4) in the context of this paper, where one of the referential spaces is finite; we refer to the works by Miranda and Zaffalon $(2015,2016)$ for a more general study of independent products. 
Definition 28 (Strong product for lower previsions). Let $\underline{P}_{\Omega}^{\prime}, \underline{P}_{\mathcal{X}}^{\prime}$ be two marginal coherent lower previsions. Their strong (independent) product is defined for every $f \in$ $\mathcal{L}(\Omega \times \mathcal{X})$ as

$$
\underline{P}_{\Omega}^{\prime} \otimes \underline{P}_{\mathcal{X}}^{\prime}(f):=\min \left\{P_{\Omega}^{\prime}\left(P_{\mathcal{X}}^{\prime}(f \mid \Omega)\right): P_{\Omega}^{\prime} \geq \underline{P}_{\Omega}^{\prime}, P_{\mathcal{X}}^{\prime} \geq \underline{P}_{\mathcal{X}}^{\prime} \text { linear previsions }\right\},
$$

where

$$
P_{\mathcal{X}}(\cdot \mid \Omega):=\sum_{\omega \in \Omega} I_{\{\omega\}} P(\cdot \mid\{\omega\}) \quad \text { and }(\forall f \in \mathcal{L}(\Omega \times \mathcal{X})) P(f \mid\{\omega\}):=P_{\mathcal{X}}^{\prime}(f(\omega, \cdot)) .
$$

It is important to remark that the order in which the concatenation is made is relevant: not only we are assuming the irrelevance of $\Omega$ towards $\mathcal{X}$, and not the other way, but in general given two linear previsions $P_{1}, P_{2}$ the concatenations $P_{1}\left(P_{2}\right)$ and $P_{2}\left(P_{1}\right)$ may not coincide when one of the possibility spaces is infinite, due to the finite additivity of linear previsions; we refer to the work by de Cooman and Miranda (2009) and Miranda and Zaffalon (2015) for some discussion of this problem.

\subsubsection{State Dependent Preferences}

As in the case of desirable gambles, we define state dependence as the lack of state independence: this means that if we consider a coherent lower prevision $\underline{P}$ with marginals $\underline{P}_{\Omega}^{\prime}, \underline{P}_{\mathcal{X}}^{\prime}$, it is said to model state-dependent preferences when it does not dominate the concatenation $\underline{P}_{\Omega}^{\prime}(\underline{P}(\cdot \mid \Omega))$, where $\underline{P}(\cdot \mid \Omega)$ is derived from $\underline{P}_{\mathcal{X}}^{\prime}$ by an assumption of epistemic irrelevance.

\subsubsection{Completeness}

The definition of completeness for lower previsions is a rephrasing of that for desirable gambles:

Definition 29 (Completeness of beliefs and values for lower previsions). A coherent lower prevision $\underline{P}$ on $\mathcal{L}(\Omega \times \mathcal{X})$ is said to represent complete beliefs if its marginal $\underline{P}_{\Omega}$ is linear. It is said to represent complete values if its marginal $\underline{P}_{\mathcal{X}}$ is linear. Finally, if $\underline{P}$ is linear, then it is said to represent complete preferences.

There are a number of equivalent ways in which we can characterise the linearity of previsions in terms of the corresponding set of desirable gambles:

Proposition 15. Let $\underline{P}$ be a coherent lower prevision on $\mathcal{L}$ and $\mathcal{R}$ its corresponding coherent set of strictly desirable gambles. The following are equivalent:

(i) $\underline{P}$ is a linear prevision.

(ii) If $f \notin \mathcal{R}$ then $\varepsilon-f \in \mathcal{R}$ for all $\varepsilon>0$.

(iii) $\mathcal{R}$ is negatively additive, meaning that $f, g \notin \mathcal{R} \Rightarrow(\forall \varepsilon>0) f+g-\varepsilon \notin \mathcal{R}$.

(iv) For any event $A$, any $\alpha \in(0,1)$ and real numbers $\mu_{1}>\mu_{2}$, either $\alpha \mu_{1}+(1-\alpha) \mu_{2} \succ$ $\mu_{1} I_{A}+\mu_{2} I_{A^{c}}$ or $\mu_{1} I_{A}+\mu_{2} I_{A^{c}} \succ \alpha^{\prime} \mu_{1}+\left(1-\alpha^{\prime}\right) \mu_{2}$ for every $\alpha^{\prime}<\alpha$, where $\succ$ is the coherent preference relation on gambles associated with $\mathcal{R}$ by Definition 21. 
The equivalence of (i) and (ii) was established by Walley (1991, sec. 3.8.3). The characterisation of (i) through negative additivity in (iii) resembles the one used to deduce the completeness of values by Galaabaatar and Karni (2013) (see their Axiom A.6). The characterisation of (i) in (iv) has an analog too in the work of Galaabaatar and Karni (2013) (see their Axiom A.7), which is used there to derive the completeness of beliefs. So it is interesting to see that two notions that have been used with separate aims in the literature are actually the same notion once we represent utilities, besides probabilities, as coherent lower previsions. We can then focus on any of those formulations to immediately deduce characterisations for all the cases of completeness in preferences:

Proposition 16. Consider a coherent preference relation $\succ$ on gambles represented by a coherent lower prevision $\underline{P}$ on $\mathcal{L}(\Omega \times \mathcal{X})$, whose corresponding coherent set of gambles is denoted by $\mathcal{R}$. Let $\mathcal{R}_{\Omega}, \mathcal{R}_{\mathcal{X}}$ denote its marginals. Then:

(i) $\underline{P}$ represents complete beliefs $\Leftrightarrow \mathcal{R}_{\Omega}$ is negatively additive.

(ii) $\underline{P}$ represents complete values $\Leftrightarrow \mathcal{R}_{\mathcal{X}}$ is negatively additive.

(iii) $\underline{P}$ represents complete preferences $\Leftrightarrow \mathcal{R}$ is negatively additive.

\subsection{State Independence Means Strong Product in Former Proposals}

It is time to discuss in detail how our proposal of state independence relates to previous ones that have been made under incomplete preferences. We focus on Galaabaatar and Karni's (2013) work, which provides quite a general treatment and is easier therefore to put into correspondence with ours. They obtain state independence by means of their Axioms A.4 and A.5. The former is also called the dominance axiom in their work; the latter corresponds to Eq. (22) in the following:

Proposition 17. Let $\underline{P}$ be a coherent lower prevision on $\mathcal{L}(\Omega \times \mathcal{X})$ and let $\underline{P}_{\Omega}^{\prime}, \underline{P}_{\mathcal{X}}^{\prime}$ denote the marginals of $\underline{P}$. For any $P_{\mathcal{X}}^{\prime} \geq \underline{P}_{\mathcal{X}}^{\prime}$, we define $P_{\mathcal{X}}(\cdot \mid \Omega)$ by means of Eq. (21). Then

$$
\underline{P} \leq \underline{P}\left(P_{\mathcal{X}}(\cdot \mid \Omega)\right) \text { for every } P_{\mathcal{X}}^{\prime} \geq \underline{P}_{\mathcal{X}}^{\prime}
$$

if and only if $\underline{P} \leq \underline{P}_{\Omega} \otimes \underline{P}_{\mathcal{X}}$.

Thus, Galaabaatar and Karni's Axiom A.5 imposes that preferences be represented by a coherent lower prevision $\underline{P}$ that is pointwise at most as precise as the strong product of the marginal models. In other words, the greatest coherent lower prevision $\underline{P}$ with marginals $\underline{P}_{\Omega}^{\prime}, \underline{P}_{\mathcal{X}}^{\prime}$ satisfying $(22)$ is their strong product.

Next we give an alternative characterisation of the strong product as the least informative model satisfying (our reformulation of) Galaabaatar and Karni's Axiom A.4. For every $\omega \in \Omega$ and $f \in \mathcal{L}(\Omega \times \mathcal{X})$, let us define the $\mathcal{X}$-measurable gamble

$$
\begin{aligned}
f^{\omega}: \Omega \times \mathcal{X} & \rightarrow \mathbb{R} \\
\left(\omega^{\prime}, x\right) & \mapsto f(\omega, x) .
\end{aligned}
$$

The reformulation of their axiom is the following: 
STP. $(\forall g, f \in \mathcal{L}(\Omega \times \mathcal{X}))\left((\forall \omega \in \Omega) \underline{P}\left(g-f^{\omega}\right) \geq 0 \Rightarrow \underline{P}(g-f) \geq 0\right)$.

This condition characterises independent products in the linear case:

Lemma 18. A linear prevision $P$ satisfies STP if and only if it is an independent product of its marginals. Moreover, STP is preserved by taking lower envelopes.

In order to extend Lemma 18 to the imprecise case, we prove that (i) the result holds when both $\Omega, \mathcal{X}$ are finite, and that (ii) the case of infinite $\Omega$ can be approximated as a limit of finite sets. The next proposition addresses the first case.

Proposition 19. Let $\Omega, \mathcal{X}$ be finite spaces, and let $\underline{P}$ be a coherent lower prevision on $\mathcal{L}(\Omega \times \mathcal{X})$. Then $\underline{P}$ satisfies STP if and only if it is a lower envelope of linear previsions satisfying

$$
(\forall \omega \in \Omega)(\forall x \in \mathcal{X}) P(\{(\omega, x)\})=P(\{\omega\}) P(\{x\}) .
$$

It is an open problem at this stage whether a result akin to Proposition 19 holds when one of the spaces is infinite.

The second case to be dealt with is done through the next result:

Proposition 20. Consider a coherent lower prevision $\underline{P}$ on $\mathcal{L}(\Omega \times \mathcal{X})$ with marginals $\underline{P}_{\Omega}^{\prime}, \underline{P}_{\mathcal{X}}^{\prime}$. If $\underline{P}$ satisfies $\mathrm{STP}$, then it dominates the strong product $\underline{P}_{\Omega}^{\prime} \otimes \underline{P}_{\mathcal{X}}^{\prime}$.

We see then that the strong product is the smallest (i.e., most conservative) coherent lower prevision with given marginals that satisfies STP.

We have thus two characterisations of the strong product. Each of them can be used to characterise stochastic independence in the case of complete preferences:

Corollary 21. Let $P$ be a linear prevision on $\mathcal{L}(\Omega \times \mathcal{X})$. The following are equivalent:

(i) $P$ is the product of its marginals.

(ii) P satisfies (22).

(iii) P satisfies STP.

Note that the corollary holds also for the case where $\Omega$ is infinite.

Most importantly, if we put the two conditions together, then we can characterise the strong product:

Theorem 22. Let $\underline{P}$ be a coherent lower prevision on $\mathcal{L}(\Omega \times \mathcal{X})$. Then $\underline{P}$ is the strong product of its marginals if and only if it satisfies (22) and STP.

What we have obtained is that the notion of state independence is implicitly implemented by Galaabaatar and Karni (2013) in terms of the strong product. We find this interesting for several reasons:

- It provides a bridge to a well known notion of independence with sets of probabilities.

- It shows that Axioms A.4 and A.5, used by Galaabaatar and Karni (2013) to formalise state independence, can be employed also when $\Omega$ is infinite. 
- It implies that there are weaker and potentially better notions than the traditional formulation of state independence, such as the weakest independent products of Sections 5.1.1 and 5.2.1. One reason for this is, for instance, that the strong product may not exist when both $\Omega, \mathcal{X}$ are infinite, as shown by Miranda and Zaffalon (2015, Ex. 1). Some alternatives in this case are discussed in Section 5 of the same paper.

- Turning the viewpoint around, it provides a behavioural interpretation of the strong product (so far, the strong product appears to have been justified only through sensitivity analysis), in particular by regarding it as the weakest model that satisfies STP. This is a behavioural interpretation as it characterises the strong product without resorting to precise models of probability and utility (as it would be done using sensitivity analysis); it instead defines it by only requiring some gambles to be desirable once others are. Note that this behavioural interpretation readily applies to the traditional use of the strong product in the case of sets of probabilities (when no utility - or, better, only linear utility - is considered): the reason is that there is nothing in STP that restricts its application to the case of probability and utility (it is enough to reinterpret $\mathcal{X}$ as a further possibility space besides $\Omega$ ).

\section{Related Work}

In the next sections, we compare our work with three main approaches in the literature that have dealt with the axiomatisation of incomplete preferences.

\subsection{The Work of Galaabaatar and Karni}

The work most influential for this paper was carried out by Galaabaatar and Karni (2013) (GK). They provide an axiomatisation of incomplete preferences for finite spaces of possibilities and outcomes. They consider a strict preference ordering on acts and use axioms Axioms A.1-A.3 that are essentially ours as well (with the difference that they impose Archimedeanity all the way through). In fact, the similarity of the approaches has allowed us to exploit some of their results in key passages of our Theorem 8 and Proposition 19.

A preliminary difference with our work is at the level of assumptions: in fact, GK assume that a preference relation is bounded by worst and best acts while ours only needs the former. Moreover, the assumption about the worst act is not without consequences in their case: as we have shown in Section 3.3, if we start with a coherent preference relation without a worst act, we can always extend it to one with a worst outcome, but the minimal extension will never satisfy the Archimedean property A3 (except in the trivial case where we start with a vacuous preference relation; see Theorem 9); and this Archimedean condition is needed throughout all of GK's paper.

Galaabaatar and Karni (2013, Thm. 1) show that a coherent and Archimedean preference relation that is moreover bounded and satisfies their so-called dominance axiom is in correspondence with a family $\Phi$ of probability-utility models. They represent these as $\left\{\left(\Pi^{U}, U\right): U \in \mathcal{U}\right\}$, where $\Pi^{U}$ denotes the family of probability measures on $\Omega$ that is combined with a particular utility function $U$ on $\mathcal{X}$. Then Galaabaatar and Karni (2013, Thm. 2) provide conditions for the complete separation of probabilities and utilities, so that $\Pi^{U}$ does not depend on $U$ in the above representation. This is implicitly done using the 
strong product by GK. We make this explicit with Theorem 22, which actually also holds for infinite $\Omega$. Moreover, we propose weaker and arguably more realistic models of state independence, such as the marginal extension models of Propositions 12 and 14.

Finally, GK also discuss the cases of precise probabilities or utilities. Their work has actually been connected to other characterisations of linear previsions in Proposition 15.

Galaabaatar and Karni (2013, Thm. 3) characterise Knightian uncertainty, that is, imprecise probabilities and precise utilities. To this end, they add an axiom of negative transitivity on constant acts on $\Omega$. We have established an analogous result in our context in Proposition 16(ii) using $\mathcal{X}$-measurable gambles and the negative additivity of a coherent set of desirable gambles.

Concerning precise probabilities and imprecise utilities, the characterisation established by Galaabaatar and Karni (2013, Thm. 4) requires their Axiom A.7 that is analogous to condition (iii) of Proposition 16; in our case it becomes another axiom of negative additivity, in a uniform way with respect to Knightian uncertainty.

Note that in both cases our results are established also for infinite $\Omega$.

\subsection{The Work of Nau}

Another important work in the axiomatisation of the expected utility model with imprecise probabilities and utilities was carried out by Nau (2006). Like GK, he considers the case of finite spaces of possibilities and outcomes; unlike them, he axiomatises weak preference, that is, he uses a reflexive relation $\succeq$, instead of strict preference $\succ$ as we have considered in this paper. He discusses this point in some detail (see Nau, 2006, sec. 4).

From the point of view of desirability, Nau works with sets of almost-desirable gambles, that is, with closed convex cones (see Remark 1). Those models are equivalent to sets of expectations and hence they cannot deal with non-Archimedean situations. However, it is worth noting that it is possible to model weak preference through desirability while retaining the capability to deal with non-Archimedean problems: it suffices to employ a formulation of general desirability that is essentially equivalent to D1-D4 but that assumes the zero gamble to be desirable (see Walley, 1991, app. F, Axioms D2, D3, D10, D11). This means that the present paper could be rewritten with minor changes so as to model weak preference while maintaining the same level of generality.

Nau imposes the existence of a best and a worst outcome (similarly to GK, with the difference that they impose best and worst acts); we refer to the previous section for a discussion of this point. In addition, he also requires that the relation $\succeq$ satisfies a continuity axiom. Nau (2006, Thm. 2) proves that under these conditions, and together with the analogue of axioms A1, A2 in case of weak preference, the relation $\succeq$ can be represented by means of a family of state-dependent expected utility functions. This result is similar to our basic representation theorem in terms of a coherent set of desirable gambles, or its associated coherent lower prevision $\underline{P}$ : in the finite case any linear prevision $P \in \mathcal{M}(\underline{P})$ will be equal to $P(P(\cdot \mid \Omega))$, by marginal extension - the law of total probability - , so that we can regard it in general as a state-dependent representation (even though for the special cases made of stochastic products, this will obviously be formal rather than substantial).

With respect to state independence, he studies under which conditions the relation $\succeq$ is characterised by means of a family of state-independent models, which in the language 
of this paper correspond to factorising linear previsions. Nau (2006, Thm. 3) first obtains a representation of this type for constant von Neumann-Morgenstern lotteries only, and then another representation for arbitrary horse lotteries in his Theorem 4, by means of the following axiom:

$$
\begin{aligned}
& \text { If } p \succ q, A \succeq \alpha b+(1-\alpha) z, \text { and } \beta b+(1-\beta) z \succeq B \text {, then: } \\
& \alpha^{\prime} A p+\left(1-\alpha^{\prime}\right) p^{\prime} \succeq \alpha^{\prime} A q+\left(1-\alpha^{\prime}\right) q^{\prime} \Rightarrow \beta^{\prime} B p+\left(1-\beta^{\prime}\right) p^{\prime} \succeq \beta^{\prime} B q+\left(1-\beta^{\prime}\right) q^{\prime},
\end{aligned}
$$

where $b$ denotes the best act, $A, B$ are subsets of $\Omega, \alpha>0, f^{\prime}, g^{\prime}$ are constant acts and $\alpha^{\prime}, \beta^{\prime}$ are related by the formula

$$
\alpha^{\prime}=1 \Rightarrow \beta^{\prime}=1 \text { and } \alpha^{\prime}<1 \Rightarrow \frac{\beta^{\prime}}{1-\beta^{\prime}} \leq \frac{\alpha \alpha^{\prime}}{(1-\alpha)\left(1-\alpha^{\prime}\right)}
$$

He calls this axiom strong state-independence. In our language, the representation he obtains means that the corresponding lower prevision is a lower envelope of factorising linear previsions. It has been characterised in Proposition 19 by means of the somewhat simpler axiom STP. One advantage of Nau's axiom, though, is its constructive character, in the sense that it allows us to establish new preferences if we have three preferences in the manner depicted above, whereas axiom STP requires the comparison of $g$ with $f^{\omega}$ for all $\omega$ in the (potentially infinite) set $\Omega$.

Nau (2016) also studies the problem of axiomatising incomplete preferences (see also Nau, 2008). In addition to the results discussed above, he also tackles a number of issues we have not considered in this paper, such as the epistemic differences between the axiomatisations of (imprecise) subjective probability and expected utility; the technical role of separation theorems and duality in the axiomatisations; and the importance of a no arbitrage condition as the main rationality criterion, amongst other things.

\subsection{The Work of Seidenfeld, Schervish and Kadane}

Seidenfeld, Schervish, and Kadane (1995) (SSK) study the problem for strict preference orders and impose on them Axioms A1 and A2, such as GK and we do. However, they allow for a countable space of prizes $\mathcal{X}$ and for simple lotteries defined as $\sigma$-additive probabilities on such a countable support. The space of possibilities $\Omega$ can be regarded as finite in their approach. Moreover, they use convex sets of expected utilities that take advantage of the border to enrich the expressiveness of the model.

Their approach can be understood somehow as sort of dual to ours: we use infinite $\Omega$ and finite $\mathcal{X}$; we also take advantage of the border, but in our case it is the border of the cone. And yet their focus on sets of probabilities rather than cones of gambles seems to make the treatment more difficult: for instance, they have to use transfinite induction instead of separation theorems; they are prevented from defining state independence in general (only a notion of 'almost' state independence is allowed); they cannot use the Archimedean condition A3, since it is not suited to deal with sets of expected utilities that are informative on the border, and rather use:

tA3. $(\forall n \in \mathbb{N})\left(p_{n} \succ q_{n}\right)\left(\left(p_{n}\right)_{n \in \mathbb{N}} \rightarrow p\right)\left(\left(q_{n}\right)_{n \in \mathbb{N}} \rightarrow q\right)$ $\Rightarrow(\forall r \in \mathcal{H})(r \succ p \Rightarrow r \succ q)(q \succ r \Rightarrow p \succ r)$, 
where the convergence of the horse lotteries is understood as pointwise.

Since SSK's relation $\succ$ is coherent for us, it corresponds to a coherent set $\mathcal{R}$ of desirable gambles. Moreover, that $\left(p_{n}\right)_{n \in \mathbb{N}} \rightarrow p$ and $\left(q_{n}\right)_{n \in \mathbb{N}} \rightarrow q$ imply that $\left(p_{n}-q_{n}\right)_{n \in \mathbb{N}} \rightarrow(p-q)$; whence, if $p_{n} \succ q_{n}$ for every $n \in \mathbb{N}, \psi(p-q)$ is in the topological closure $\overline{\mathcal{R}}$ of $\mathcal{R}$. This means that Axiom tA3 can be reformulated as ${ }^{20}$

tA3'. $f \in \overline{\mathcal{R}}, g \in \mathcal{R} \Rightarrow f+g \in \mathcal{R}$.

We can use this desirability formulation of tA3 to give an example of a minimal extension to a worst outcome of a relation satisfying axioms A1, A2, tA3. This is in fact known to be possible in SSK's setting as it allows for open sets of probabilities.

Example 1. Let $\underline{P}$ be a coherent lower prevision on $\mathcal{L}(\Omega \times \mathcal{X})$ such that $\underline{P}(\{(\omega, x)\})>0$ for every $(\omega, x) \in \Omega \times \mathcal{X}$, and consider the relation $\succ^{\prime}$ on $\mathcal{H} \times \mathcal{H}$ given by $r \succ^{\prime} s \Leftrightarrow \underline{P}(r-s)>0$. This relation satisfies axioms $\mathrm{A} 1, \mathrm{~A} 2$ and $\mathrm{tA} 3$. Let the relation $\succ$ on $\mathcal{H}_{z} \times \mathcal{H}_{z}$ be the minimal extension of the relation $\succ^{\prime}$ to the worst outcome that satisfies axioms $\mathrm{A} 1, \mathrm{~A} 2$, as given by Theorem 9.

Then $p \succ q$ implies $\underline{P}(\psi(p-q))>0$. To prove this, note that if $p \triangleright q$, then $\psi(p-q) \in \mathcal{L}^{+}$, and then given $(\omega, x) \in \Omega \times \mathcal{X}$ such that $\psi(p-q)(\omega, x)>0$, it holds that $\underline{P}(\psi(p-q)) \geq$ $\underline{P}\left(I_{\{(\omega, x)\}} \psi(p-q)(\omega, x)\right)>0$. On the other hand, if $\psi(p-q)=r-s$ for some $r, s \in \mathcal{H}, r \succ^{\prime} s$, then $\underline{P}(\psi(p-q))=\underline{P}(r-s)>0$.

It follows that given a gamble $f$ that is the limit of a sequence of gambles of the type $p_{n}-q_{n}, n \in \mathbb{N}$, with $p_{n} \succ q_{n}$, it holds that $\underline{P}(\psi(f)) \geq 0$. This and the super-additivity of $\underline{P}$ imply $\mathrm{tA} 3^{\prime}$; and as a consequence the relation $\succ$ satisfies tA3. $\diamond$

\section{Discussion and Conclusions}

In this paper we have formally bridged two different theories: the decision-theoretic axiomatisation of incomplete preferences and Williams' theory of desirability - as extended here to vector-valued gambles.

\subsection{Main Message}

This bridge has given us a fresh new perspective on the axiomatisation of incomplete preferences. Our view is that this perspective enables streamlining the question and unifying several of its aspects, while maintaining a generality that allows us to deal with nonArchimedean problems as well as infinite possibility spaces. To pinpoint the question in different words, the present paper allows us to replace (quite a general version of) the usual axioms of rational preferences that can be found in textbooks with D1-D4, which, for one thing, we find simpler to understand. Most importantly, and somewhat differently from the traditional axiomatisation, D1-D4 directly lead to operators to manipulate the theory at the very level of cones, for conditioning, marginalisation, etcetera; axioms and operators are nicely close in desirability.

20. Interestingly, a similar condition has appeared with the name weak desirability in the work by de Cooman and Quaeghebeur (2012). Nevertheless, what tA3' means is that any almost-desirable gamble is also weakly desirable, and not only the opposite; see also app. A2 by Miranda and Zaffalon (2010). A similar condition also appears in the broader framework of sets of accepted-rejected statements with the name of favouring sweetened deals in a paper by Quaeghebeur et al. (2015). 
Our results have implications for imprecise probability as well. Most importantly, the extension of desirability we have proposed here to vector-valued gambles can be regarded as the foundation of a very general theory of uncertainty and decision making capable of dealing with imprecise non-linear utility. This is guaranteed by the equivalence that we have established between desirability and incomplete preferences; in practice, it is immediately achieved by the simple move of redefining gambles on the product space $\Omega \times \mathcal{X}$. In hindsight, it is surprising that such a simple, and yet powerful, change has had to wait so long to be found.

Let us stress once more, as we have pointed out in a number of places in the paper, that we are not claiming to be the first ones relating the axiomatisation of incomplete preferences with that of some types of cones of gambles. But we appear to be the first ones making the explicit link to desirability theory, with the presented type of generality, and rigorously obtaining from this a number of new results.

\subsection{Summary of the Results Following from the Equivalence}

In our opinion, the main contributions that we can derive from our equivalence are related to two categories: the Archimedeanity axiom and the decomposition and completeness of preferences.

\subsubsection{ARChIMEDEANITY}

We have shown in Section 3 that the traditional Archimedean axiom conflicts with the possibility to represent a maximally uninformative, or vacuous, relation. By defining a weak Archimedean condition, we have solved the problem while maintaining the ability to represent preferences in terms of sets of expected utilities.

Weak Archimedeanity allows also the probabilistic models derived in our representation to be meaningfully defined on uncountable sets, unlike in the case of the traditional Archimedean axiom. This is remarked in Section 4.

In Section 3.3 we have shown that a coherent preference relation that has no worst act can always be extended in a least-committal way to one that has it. But the extension is never (weakly) Archimedean irrespectively of the preference relation we start from-apart from the case where this relation is empty. Moreover, the notion of minimal extension is ill-defined in the Archimedean case: given any Archimedean extension, it is always possible to find a smaller one.

In Appendix A.1 we dive deeper into this problem; we define a strong Archimedean condition and show that the weak, strong and traditional Archimedean conditions are all (essentially) equivalent in case a relation has a worst outcome. When it has not, and restricting the attention to the case of finite $\Omega$, in Appendix A.2 we show that the strong Archimedean condition leads to an Archimedean extension and that instead the traditional one does not serve the purpose. Moreover, we show that strong Archimedeanity is equivalent to the topological openness of the cone $\mathcal{C}$.

Finally, in Appendix A.3 we give a definition of 'full Archimedeanity' that captures all and only the problems that can be expressed with collections of sets of expected utilities, and of which the Archimedean condition is a special case. 


\subsubsection{Decomposition and Completeness of Preferences}

In Section 5.1, we have discussed the cases of state dependence and independence directly for the case of desirable gambles - whence also for non-Archimedean problems; this seems to be done here for the first time. We have shown that there are much weaker (and arguably, more intuitive) notions than the traditional ones we can employ to model state independence.

Something similar happens with the case of complete preferences. The definition in the case of desirability is straightforward and very general.

In Section 5.2, we have described what happens of these notions when we move down to the level of coherent lower previsions, that is, sets of expected utilities. Also in this case, we have provided weaker notions than the traditional ones that are at the same time very direct.

For the case of complete preferences, we have given a number of equivalent conditions to impose them, thus also simplifying the traditional formulations, and have shown that we can use the very same condition both for the case of complete beliefs and incomplete values and for the opposite one (the so-called Knightian uncertainty).

In Section 5.3 we have considered two axioms used in the literature to impose state independence in the case of a multiprior expected multiutility representation. After quite an involved analysis, we have shown that imposing those axioms is equivalent to modelling state independence with sets of expectations using the strong product. This gives the strong product, for the first time, a behavioural interpretation.

\subsection{Future Research}

With respect to future research, it will be important to relax the current limitation that prizes $\mathcal{X}$ be finite in number. Although some of our results can easily be formulated in the case where $\mathcal{X}$ is countable, this is not the case for those related to state dependence and independence, because when both $\Omega, \mathcal{X}$ are infinite a number of mathematical complications arise; we refer to a paper by Miranda and Zaffalon (2015) for a discussion of this point. More generally speaking, allowing for infinitely many outcomes is challenging especially in the uncountable case, as we cannot represent the simple lottery $p(\omega, \cdot)$ through a countably additive probability when $\mathcal{X}$ is uncountable, and using a finitely additive probability does not seem to be immediate. Still, this case has received quite some attention in the literature; see the work by Ok et al. (2012), Dubra et al. (2004), del Amo and Ríos Insua (2002) and Ghirardato and Siniscalchi (2012), just to name a few.

Another question concerns conglomerability. We have tried to minimise its involvement in this paper so as not to overcomplicate the analysis. And yet the issue of conglomerability remains an important one to clarify; the central question is whether or not decision making in the infinite case needs a conglomerability axiom to be considered rational.

Also, it would be important to work out the relation between this work and the growing literature on preference modelling in artificial intelligence. The general formulation established here might provide foundations for existing methods, as well as deliver a full toolset to move back and forth between preferences and expected utilities.

Finally, we hope that this work contributes to clarifying, and thus demystifying, the notion of imprecise probability: despite the unconventional language (made of terms such as previsions, gambles, desirability), now we can see that one of the most credited theories 
in the field, Williams', actually has a very familiar face: that of the classical axiomatisation of preference relations in the special case where the space of prizes has only one element; Walley's theory is the same with an additional requirement of conglomerability.

\section{Acknowledgments}

A first version of this manuscript appeared as a technical report (Zaffalon \& Miranda, 2015), which a few other papers have started referring to by now. The main content is essentially the same across that report and the present paper; however, this paper has been refined thanks to the suggestions of the following people: Robert Nau, who has commented on the report and has pointed us to his course notes on choice theory besides providing us with a draft of the first five chapters of his book (in preparation) about arbitrage and rational choice; the anonymous referees, and in particular Ref. 2 and 3, who have read the paper truly carefully providing us with many useful comments. All these people are gratefully acknowledged. This work has been partially supported by the Swiss National Science Foundation grants nos. 200021_146606 / 2, IZKSZ2_162188, 407540_167199 / 1, and by project TIN2014-59543-P.

\section{Appendix A. Strong and Full Archimedeanity}

In this section we deepen the study of Archimedeanity. In Appendix A.1 we introduce a strong notion of Archimedeanity, which we characterise together with the previous notions. Strong Archimedeanity is shown in Appendix A.2 to be equivalent to the openness of $\mathcal{C}$, and this is used to show that there are Archimedean extensions to the worst outcome in the case of finite $\Omega$. Finally, in Appendix A.3 we give a notion of 'full Archimedeanity' that captures the subclass of coherent sets of desirable gambles that are equivalent to collections of sets of linear previsions.

\section{A.1 Archimedean Conditions and Their Relations}

Let us consider a strengthening of the Archimedean property A3:

Definition 30 (Strong Archimedean condition). Let $\succ$ be a coherent preference relation on $\mathcal{H} \times \mathcal{H}$. We say that the relation is strongly Archimedean if it satisfies

sA3. $(p, q \in \mathcal{H}) p \succ q \Rightarrow(\forall r, s \in \mathcal{H})(\exists \beta \in(0,1)) \beta p+(1-\beta) r \succ \beta q+(1-\beta) s$ [strong Archimedeanity].

That sA3 is in fact stronger than the usual Archimedean property A3 is established in the following:

Proposition 23. Let $\succ$ be a coherent relation and $\mathcal{C}$ the corresponding cone. Then:

(i) Relation $\succ$ satisfies $\mathrm{A} 3 \Leftrightarrow(\forall f, g \in \mathcal{C})\left(\exists \beta_{1}, \beta_{2} \in(0,1)\right) \beta_{1} f-\left(1-\beta_{1}\right) g \in \mathcal{C}, \beta_{2} g-(1-$ $\left.\beta_{2}\right) f \in \mathcal{C}$.

(ii) Relation $\succ$ satisfies sA3 $\Leftrightarrow(\forall f \in \mathcal{C})(\forall g \in \mathcal{A})(\exists \beta \in(0,1)) \beta f+(1-\beta) g \in \mathcal{C}$. 
(iii) Relation $\succ$ satisfies $\mathrm{sA} 3 \Rightarrow$ relation $\succ$ satisfies $\mathrm{A} 3$.

To see that A3 and sA3 are not equivalent in general, consider the following example:

Example 2. Consider a gamble $0 \neq f \in \mathcal{A}$, and let us define the binary relation $\succ$ on $\mathcal{H} \times \mathcal{H}$ by $p \succ q \Leftrightarrow p-q=\lambda f$ for some $\lambda>0$. This relation satisfies the following axioms:

A1. $f \neq 0 \Rightarrow p \nsucc p$ for every $p \in \mathcal{H}$. Moreover, if $p \succ q \succ r$, there are $\lambda_{1}, \lambda_{2}>0$ such that $p-q=\lambda_{1} f$ and $q-r=\lambda_{2} f$, and then $p-r=\left(\lambda_{1}+\lambda_{2}\right) f$, whence $p \succ r$.

A2. $p \succ q \Rightarrow \exists \lambda>0$ such that $p-q=\lambda f$. Given $r \in \mathcal{H}$ and $\beta \in(0,1], \beta p+(1-\beta) r \succ$ $\beta q+(1-\beta) r$, since $\beta(p-q)=\beta \lambda f$. The converse is analogous.

A3. We apply Proposition 23. Given $f_{1}, f_{2} \in \mathcal{C}$, there are $\lambda_{1}, \lambda_{2}>0$ such that $f_{1}=\lambda_{1} f$ and $f_{2}=\lambda_{2} f$. Then there are $\beta_{1}, \beta_{2} \in(0,1)$ such that $\beta_{1} \lambda_{1}-\left(1-\beta_{1}\right) \lambda_{2}>0$ and $\beta_{2} \lambda_{2}-\left(1-\beta_{2}\right) \lambda_{1}>0$, from which we deduce that $\beta_{1} f_{1}-\left(1-\beta_{1}\right) f_{2}$ and $\beta_{2} f_{2}-\left(1-\beta_{2}\right) f_{1}$ belong to $\mathcal{C}$.

To see that sA3 may not hold, it suffices to consider $p, q, r, s \in \mathcal{H}$ such that $p \succ q$ (so $p-q=\lambda f$ for some $\lambda>0)$ and $r \nsucc s$. Then applying Proposition 23, there should be $\beta \in(0,1)$ such that $\beta(p-q)+(1-\beta)(r-s) \in \mathcal{C}$. This implies that there is some $\lambda^{\prime}>0$ such that

$$
\beta(p-q)+(1-\beta)(r-s)=\beta \lambda f+(1-\beta)(r-s)=\lambda^{\prime} f,
$$

and as a consequence there is some $\lambda^{\prime \prime}>0$ such that $r-s=\lambda^{\prime \prime} f$, a contradiction. $\diamond$

Next, assume that the relation $\succ$ contains a worst outcome. This makes all the Archimedean conditions we have introduced so far collapse into a single notion (in the case of wA3 provided that an additional assumption of positivity holds):

Proposition 24. Let $\succ$ be a coherent relation with worst outcome $z, \mathcal{C}$ the corresponding cone and $\mathcal{R}:=\psi(\mathcal{C})$. Let $\underline{P}$ be the coherent lower prevision induced by $\mathcal{R}$ through (5). Then the following are equivalent:

(i) Relation $\succ$ satisfies sA3.

(ii) Relation $\succ$ satisfies A3.

(iii) Relation $\succ$ satisfies wA3 and $\underline{P}(f)>0$ for all $f \in \mathcal{L}^{+}(\Omega \times \mathcal{X})$.

\section{A.2 Existence of Archimedean Extensions in the Finite Case}

Note that at this point we do not know yet whether there are coherent Archimedean extensions to the worst outcome of a given coherent preference relation $\succ$. In particular we would like that there were Archimedean extensions that do not introduce informative assessments, that is, assessments other than those necessary for the extension to be weakly Archimedean. We show next that these extensions may not always exist when $\Omega$ is finite; the case of infinite $\Omega$ remains an open problem.

Showing that there exists an Archimedean extension with worst outcome is equivalent to proving that it is possible to include the set $\mathcal{R}$, given in (15), in a coherent set of strictly 
desirable gambles. We are going to show that, when a coherent preference relation $\succ$ satisfies the strong Archimedean condition sA3, and $\Omega$ is indeed finite, then the set $\mathcal{R}$ of desirable gambles $\succ$ induces has a proper superset of strictly desirable gambles.

It is interesting to note that, even when we can build an open superset of $\mathcal{R}$, it could be that any such superset introduces new preferences in the original space, as the following example shows:

Example 3. Consider $\Omega:=\{\omega\}, \mathcal{X}:=\left\{x_{1}, x_{2}, x_{3}\right\}$ and let $\succ$ be the binary relation on $\mathcal{H} \times \mathcal{H}$ given by

$$
p \succ q \Leftrightarrow(\exists \lambda>0) p-q=\lambda h,
$$

where the gamble $h$ is given by $h\left(\omega, x_{1}\right):=1, h\left(\omega, x_{2}\right):=-1, h\left(\omega, x_{3}\right):=0$. Then $\succ$ is $a$ preference relation, and the set $\mathcal{C}$ it induces is given by

$$
\mathcal{C}=\{(a,-a, 0): a>0\}
$$

where the triple refers to $\left(f\left(\omega, x_{1}\right), f\left(\omega, x_{2}\right), f\left(\omega, x_{3}\right)\right)$, in order to simplify the notation. It follows from Example 2 that this preference relation satisfies axioms A1, A2 and A3, but not sA3.

Now, if $\mathcal{R}_{1}$ is a set of strictly desirable gambles that includes the set $\mathcal{R}$ determined by (15) and $\underline{P}_{1}$ is the coherent lower prevision it induces, then it must hold that $\underline{P}_{1}(f)>0$ for every $f \in \mathcal{C}$. This means that the credal set $\mathcal{M}\left(\underline{P}_{1}\right)$ is a closed subset of

$$
\mathcal{M}:=\{P:(\forall f \in \mathcal{C}) P(f)>0\}
$$

Let $P$ be a linear prevision on $\mathcal{L}(\Omega \times \mathcal{X})$, and let $p_{1}:=P\left(\left\{\omega, x_{1}\right\}\right), p_{2}:=P\left(\left\{\omega, x_{2}\right\}\right), p_{3}:=$ $P\left(\left\{\omega, x_{3}\right\}\right)$. Then it follows that $P(f)>0$ for all $f \in \mathcal{C}$ if and only if $p_{1}>p_{2}$.

Let us show now that for any closed set $\mathcal{M}\left(\underline{P}_{1}\right) \subseteq \mathcal{M}$, the set $\left\{f \in \mathcal{A}: \underline{P}_{1}(f)>0\right\}$ is a strict superset of $\mathcal{C}$, from which it will follow that the set $\mathcal{R}_{1}$ of strictly desirable gambles associated with $\underline{P}_{1}$ will induce more preferences than those encompassed by $\succ$.

Assume ex-absurdo that $\left\{f \in \mathcal{A}: \underline{P}_{1}(f)>0\right\}$ coincides with $\mathcal{C}$. Then given $\delta>0$, for the gamble $g:=(1+\delta,-1,-\delta)$ it must hold that $\underline{P}_{1}(g) \leq 0$. This implies that there is some $P \in \mathcal{M}\left(\underline{P}_{1}\right) \subseteq \mathcal{M}$ such that $0 \geq P(g)=p_{1}-p_{2}+\delta\left(p_{1}-p_{3}\right)$. Since we know that $p_{1}>p_{2}$, it must be the case that $p_{1}<p_{3}$, whence $\delta \geq \frac{p_{1}-p_{2}}{p_{3}-p_{1}}$. Moreover, since we can make the positive number $\delta$ as close to 0 as we want, this means that for every natural number $n$ there is some $P_{n}:=\left(p_{1}^{n}, p_{2}^{n}, p_{3}^{n}\right)$ in $\mathcal{M}\left(\underline{P}_{1}\right)$ with $p_{3}^{n}-p_{1}^{n}>0$ such that

$$
\frac{1}{n} \geq \frac{p_{1}^{n}-p_{2}^{n}}{p_{3}^{n}-p_{1}^{n}}
$$

But the set $\mathcal{M}\left(\underline{P}_{1}\right)$ is compact in the metric space associated with the Euclidean topology, so it is also sequentially compact. Thus, the sequence $\left(P_{n}\right)_{n \in \mathbb{N}}$ has a convergent subsequence to some $P^{\prime}:=\left(p_{1}^{\prime}, p_{2}^{\prime}, p_{3}^{\prime}\right)$. From Eq. (24) it follows that $p_{1}^{\prime}-p_{2}^{\prime}=0$, and this is a contradiction with $P^{\prime} \in \mathcal{M}$.

This shows that the set of strictly desirable gambles associated with any closed subset of $\mathcal{M}$ will include some gamble in $\mathcal{A} \backslash \mathcal{C}$ and therefore will not induce the same preferences. $\diamond$ 
One issue in the example above is that the preference relation $\succ$ satisfies A3 but not sA3. Next we study in more detail the case where sA3 holds.

First of all, when $\succ$ is strongly Archimedean, we can always guarantee the existence of an open superset of $\mathcal{R}$ :

Proposition 25. Let $\succ$ be a coherent preference relation on $\mathcal{H} \times \mathcal{H}$ satisfying sA3. Assume $\Omega$ is finite, and let $\mathcal{R}$ be given by (15). Then there is a set of strictly desirable gambles $\mathcal{R}_{1}$ that includes $\mathcal{R}$.

Moreover, under strong Archimedeanity there are cases where we can find sets of strictly desirable gambles that include $\mathcal{R}$ and that introduce no new preferences in the original space:

Example 4. Consider $\Omega:=\{\omega\}, \mathcal{X}:=\left\{x_{1}, x_{2}, x_{3}\right\}$ and let $\succ$ be the binary relation on $\mathcal{H} \times \mathcal{H}$ given by

$$
f \succ g \Leftrightarrow f\left(\omega, x_{1}\right)<g\left(\omega, x_{1}\right) \text { and } f\left(\omega, x_{2}\right)<g\left(\omega, x_{2}\right) .
$$

Then $\succ$ is a coherent preference relation, and the set $\mathcal{C}$ it induces is given by

$$
\mathcal{C}=\{(a, b,-a-b): \max \{a, b\}<0\},
$$

where again the triple refers to $\left(f\left(\omega, x_{1}\right), f\left(\omega, x_{2}\right), f\left(\omega, x_{3}\right)\right)$. Now, if $\mathcal{R}_{1}$ is a set of strictly desirable gambles that includes the set $\mathcal{R}$ determined by (15) and $\underline{P}_{1}$ is the coherent lower prevision it induces, then it must hold that $\underline{P}_{1}(f)>0$ for every $f \in \mathcal{C}$. This means that the credal set $\mathcal{M}\left(\underline{P}_{1}\right)$ is a closed subset of

$$
\mathcal{M}:=\{P:(\forall f \in \mathcal{C}) P(f)>0\} .
$$

Let $P$ be a linear prevision on $\Omega \times \mathcal{X}$, and let $p_{1}:=P\left(\left\{\omega, x_{1}\right\}\right), p_{2}:=P\left(\left\{\omega, x_{2}\right\}\right)$. Then for every $a, b \in \mathbb{R}$, if we take $f$ on $\Omega \times \mathcal{X}$ given by $f\left(\omega, x_{1}\right):=a, f\left(\omega, x_{2}\right):=b, f\left(\omega, x_{3}\right):=-a-b$, it holds that $P(f)=a\left(2 p_{1}+p_{2}-1\right)+b\left(2 p_{2}+p_{1}-1\right)$. From this it follows that $P \in \mathcal{M}$ if and only if

$$
\min \left\{2 p_{1}+p_{2}-1,2 p_{2}+p_{1}-1\right\}<0 \text { and } \max \left\{2 p_{1}+p_{2}-1,2 p_{2}+p_{1}-1\right\} \leq 0 .
$$

Let us show now that there is a closed convex set $\mathcal{M}\left(\underline{P}_{1}\right) \subseteq \mathcal{M}$ such that the set $\{f \in$ $\left.\mathcal{A}: \underline{P}_{1}(f)>0\right\}$ agrees with $\mathcal{C}$, from which it will follow that the set $\mathcal{R}_{1}$ of strictly desirable gambles associated with $\underline{P}_{1}$ induces the same preferences on the original space as the relation $\succ$.

To see that this is indeed the case, consider $Q_{1}:=\left(q_{1}^{1}, q_{2}^{1}, 1-q_{1}^{1}-q_{2}^{1}\right), Q_{2}:=\left(q_{1}^{2}, q_{2}^{2}, 1-\right.$ $\left.q_{1}^{2}-q_{2}^{2}\right)$ in $\mathcal{M}$ such that

$$
2 q_{1}^{1}+q_{2}^{1}-1<0=2 q_{2}^{1}+q_{1}^{1}-1 \text { and } 2 q_{2}^{2}+q_{1}^{2}-1<0=2 q_{1}^{2}+q_{2}^{2}-1 .
$$

Then given $f:=(a, b,-a-b) \in \mathcal{A} \backslash \mathcal{C}$, it holds that $\max \{a, b\} \geq 0$. Then

$$
\begin{aligned}
& a \geq 0 \Rightarrow Q_{1}(f)=a\left(2 q_{1}^{1}+q_{2}^{1}-1\right) \leq 0, \\
& b \geq 0 \Rightarrow Q_{2}(f)=b\left(2 q_{2}^{2}+q_{1}^{2}-1\right) \leq 0,
\end{aligned}
$$

and as a consequence given $\underline{P}_{1}:=\min \left\{Q_{1}, Q_{2}\right\}$, it holds that $\underline{P}_{1}(f) \leq 0$ for every $f \in \mathcal{A} \backslash \mathcal{C}$. Thus, $\mathcal{M}\left(\underline{P}_{1}\right)$ is a closed convex subset of $\mathcal{M}$ satisfying that $\left\{f \in \mathcal{A}: \underline{P}_{1}(f)>0\right\}=\mathcal{C}$. $\diamond$ 
It is an open problem at this stage to determine if this is the case for any coherent preference relation that is strongly Archimedean. Our conjecture is that this will hold, because of the following result:

Proposition 26. Assume $\Omega$ is finite, and let $\succ$ be a coherent preference relation on $\mathcal{H} \times \mathcal{H}$. Then $\succ$ satisfies $\mathrm{sA} 3$ if and only if $\mathcal{C}$ is an open subset of $\mathcal{A}$.

\section{A.3 The Full Archimedean Case}

We know from Section 3 that the case of weakly Archimedean preferences is equivalent to that of a coherent set of strictly desirable gambles $\mathcal{R} \subseteq \mathcal{L}(\Omega \times \mathcal{X})$. Taking into account the correspondence between strict desirability and lower previsions, we deduce that Archimedean preferences can be equivalently represented by means of a set of linear previsions.

Despite the usefulness of this outcome, there are important limitations it is subject to. To see this, assume that there is $\omega \in \Omega$ such that $\underline{P}(\{\omega\})=0$ (it will often be the case even that $\bar{P}(\{\omega\})=0$ if $\Omega$ is infinite). Then conditioning, in the least-committal way, $\underline{P}$ on $\omega$ will lead to the vacuous model. In other words, it turns out that the very assumption to get to know $\omega$ renders the utilities vacuous. The problem here is that $\underline{P}$ is too much of an uninformative model to be able to represent non-vacuous conditionals in the presence of conditioning events with zero probability.

And yet, it is entirely possible that (unconditional) preferences are represented by a coherent lower prevision $\underline{P}$ on $\mathcal{L}(\Omega \times \mathcal{X})$, and at the same time that the utility model conditional on a state of nature $\omega$ is given by a conditional lower prevision $\underline{P}(\cdot \mid\{\omega\})$ that is different from the conditional natural extension of $\underline{P}$ given $\omega$ (see Walley, 1991, app. F4). This is to say that despite a pair such as $\underline{P}, \underline{P}(\cdot \mid \Omega)$ is only made up of probabilities and utilities, there is no single coherent lower prevision that is equivalent to it; that is, there is no set of strictly desirable gambles on $\mathcal{L}(\Omega \times \mathcal{X})$ that is equivalent to it, and hence there is no weakly Archimedean relation either. What we argue, in other words, is that there are useful preference relations, which are expressed only via (a collection of) sets of linear previsions, that are not characterised by Axiom wA3 and therefore that the Archimedean axiom is too restrictive.

If we focus the attention on the case that $\Omega$ is finite, we immediately have a characterisation of the preference relations that can be assessed by relying only on sets of linear previsions:

Definition 31 (Fully Archimedean preferences-finite $\Omega$ ). Given a coherent preference relation $\succ$ on $\mathcal{H}_{z} \times \mathcal{H}_{z}$, with $\Omega$ finite, we say that the relation $\succ$ is fully Archimedean if the corresponding coherent set of desirable gambles $\mathcal{R} \subseteq \mathcal{L}(\Omega \times \mathcal{X})$ satisfies the following condition:

$$
f \in \mathcal{R} \Rightarrow(\exists \varepsilon>0) I_{S(f)}(f-\varepsilon) \in \mathcal{R},
$$

where $S(f):=\{(\omega, x) \in \Omega \times \mathcal{X}: f(\omega, x) \neq 0\}$ is the support of $f$.

In fact, it has been shown by Miranda and Zaffalon (2010, Thm. 15) that a coherent set of desirable gambles $\mathcal{R}$ satisfies (25) if and only if it is equivalent to a collection of separately coherent conditional lower previsions. The remaining coherent sets of desirable 
gambles, those that do not satisfy (25), are in a sense the 'purest' non-Archimedean sets, since there is no way to represent them equivalently by collections of closed convex sets of linear previsions.

Note that Eq. (25) can be interpreted quite simply: what is required there is a property analogous to strict desirability but extended to the conditional case, for all events that are supports of gambles in $\mathcal{R}$. In other words, Eq. (25) is still a continuity property like the Archimedean one, but it is extended so as to be satisfied by all the relevant conditional inferences, not only the unconditional ones, as in the case of wA3.

It is possible to generalise (25) to the case of infinite $\Omega$. In order to do this, we must introduce two additional notions from the theory of desirability: we say that a set of gambles $\mathcal{R}^{\prime}$ avoids partial loss when it is included in some coherent set of gambles. The smallest such set is called its natural extension, and it is given by $\operatorname{posi}\left(\mathcal{R}^{\prime} \cup \mathcal{L}^{+}\right)$, where posi denotes the conic hull operator. The natural extension models the behavioural implications of the assessments present in $\mathcal{R}^{\prime}$ and the axioms of desirability (for more information, we refer to Augustin et al., 2014, ch. 1 and to Walley, 1991, sec. 3.7).

Definition 32 (Full strict desirability). Let $\mathcal{R}$ be a coherent set of gambles. We say that it is fully strictly desirable if it is the natural extension of a set of gambles $\mathcal{R}^{\prime}$ satisfying Eq. (25).

The term above means that $\mathcal{R}$ keeps the same information as a set of conditional lower previsions. This will be a consequence of the following result:

Proposition 27. Consider a coherent set of desirable gambles $\mathcal{R}$ in $\mathcal{L}(\Omega \times \mathcal{X})$. Let

$$
\begin{aligned}
& \mathcal{R}_{1} \mid B:=\left\{f \in \mathcal{R}: f=B f, \inf _{S(f)} f>0\right\} \cup\{0\}, \\
& \mathcal{R}_{2} \mid B:=\{f \in \mathcal{R}: f=B f,(\exists \varepsilon>0) B(f-\varepsilon) \in \mathcal{R}\}, \\
& \widetilde{\mathcal{R}} \mid B:=\left\{f+g: f \in \mathcal{R}_{1}\left|B, g \in \mathcal{R}_{2}\right| B\right\},
\end{aligned}
$$

for every set $B \subseteq \Omega \times \mathcal{X}$, and

$$
\widetilde{\mathcal{R}}:=\cup_{B \subseteq \Omega \times \mathcal{X} \widetilde{\mathcal{R}} \mid B .}
$$

Then $\mathcal{R}$ is the natural extension of $\widetilde{\mathcal{R}}$ if and only if $\mathcal{R}$ is fully strictly desirable.

As a consequence, we have the following:

Proposition 28. Let $\left(\underline{P}\left(\cdot \mid B_{i}\right)\right)_{i \in I}$ be a family of separately coherent conditional lower previsions satisfying

$$
(\forall J \subseteq I,|J|<+\infty)(\forall j \in J)\left(\forall f_{j} \in \mathcal{L}(\Omega \times \mathcal{X})\right) \sup _{\omega \in \cup_{j \in J} B_{j}}\left[\sum_{j \in J} B_{j}\left(f_{j}-\underline{P}\left(f_{j} \mid B_{j}\right)\right)\right](\omega) \geq 0,
$$

and let $\mathcal{R}$ be the natural extension of $\cup_{i \in I} \widetilde{\mathcal{R}} \mid B_{i}$, where

$$
\begin{aligned}
& \mathcal{R}_{1} \mid B_{i}:=\left\{B_{i} f: \inf _{S(f)} f>0\right\} \cup\{0\}, \\
& \mathcal{R}_{2} \mid B_{i}:=\left\{B_{i}\left(f-\underline{P}_{i}\left(f \mid B_{i}\right)+\varepsilon\right): f \in \mathcal{L}, \varepsilon>0\right\}, \\
& \widetilde{\mathcal{R}} \mid B_{i}:=\left\{f_{1}+f_{2}: f_{1} \in \mathcal{R}_{1}\left|B_{i}, f_{2} \in \mathcal{R}_{2}\right| B_{i}\right\} .
\end{aligned}
$$

Then $\mathcal{R}$ is a fully strictly desirable set of gambles. 
In other words, if a family of coherent conditional lower previsions satisfies the regularity condition (27), then there is always a fully strictly desirable coherent set that is equally expressive to them, in the sense that each inference that we can do from the family of conditionals can be done from the set, and vice versa. As a consequence, Proposition 28 generalises Thm. 15 by Miranda and Zaffalon (2010).

Let us show next that for finite possibility spaces, Definition 32 can be simplified:

Proposition 29. If $\Omega$ is finite, then the natural extension of a set of gambles satisfying Eq. (25) also satisfies (25). As a consequence, a coherent set $\mathcal{R}$ is fully strictly desirable if and only if it satisfies (25).

This result does not hold in general for infinite spaces:

Example 5. Let our possibility space be the set of natural numbers, let $B_{n}:=\{2 n-1,2 n\}$ for $n \geq 1$, and let $P\left(\cdot \mid B_{n}\right)$ be the uniform probability distribution. Consider the set of gambles $\mathcal{R}^{\prime}:=\cup_{n \in \mathbb{N}} \mathcal{R} \mid B_{n}$, with $\mathcal{R} \mid B_{n}:=\left\{f \in \mathcal{L}^{+}: f=B_{n} f\right\} \cup\left\{B_{n}\left(f-\left(\underline{P}\left(f \mid B_{n}\right)-\varepsilon\right)\right)\right.$ : $\varepsilon>0, f \in \mathcal{L}\}$. It is easy to prove that $\mathcal{R}^{\prime}$ satisfies condition (25). Its natural extension is given by $\mathcal{R}:=\operatorname{posi}\left(\mathcal{L}^{+} \cup \cup_{n \in \mathbb{N}} \mathcal{R} \mid B_{n}\right)$, which is equal to

$$
\left\{f \neq 0:(\exists J \subseteq \mathbb{N} \text { finite })\left((\forall j \in J)\left(\underline{P}\left(f \mid B_{j}\right)>0\right) \text { and }(\forall n \notin J)\left(B_{n} f \geq 0\right)\right)\right\} .
$$

To see that $\mathcal{R}$ does not satisfy Eq. (25), consider the gamble $f$ given by $f(1):=-1, f(2):=$ $2, f(n):=\frac{1}{n}$ for every $n \geq 3$. It follows from Eq. (28) that $f \in \mathcal{R}$, considering $J=\{1\}$. However, there is no $\varepsilon>0$ such that $f-\varepsilon$ belongs to $\mathcal{R}$, because for every $\varepsilon>0$ there is some natural number $n_{\varepsilon}$ such that $(f-\varepsilon)(m)<0$ for all $m \geq n_{\varepsilon}$. $\diamond$

To summarise this section, what we claim is that it could be worth considering full strict desirability as a replacement of the traditional Archimedean condition (A3 or wA3), given that it fully characterises all the problems that can be expressed only through probabilities and utilities.

\section{Appendix B. Proofs}

In this appendix we collect the proofs of all the results in the paper as well as a few results that are more technical and are needed for some proofs.

Proof of Lemma 2. Suppose ex-absurdo that there is $\bar{\omega} \in \Omega$ such that for no $x \in \mathcal{X}$, $w(\bar{\omega}, x)=1$. Then there must be $x_{1}, x_{2} \in \mathcal{X}$ such that $w\left(\bar{\omega}, x_{1}\right), w\left(\omega, x_{2}\right) \in(0,1)$. Consider the positive real $\varepsilon:=\frac{1}{2} \min \left\{w\left(\bar{\omega}, x_{1}\right), w\left(\bar{\omega}, x_{2}\right), 1-w\left(\bar{\omega}, x_{1}\right), 1-w\left(\bar{\omega}, x_{2}\right)\right\}$. Define acts $p, q$ so that they coincide with $w$ for all $\omega \in \Omega, \omega \neq \bar{\omega}$, while in $\bar{\omega}$ they are defined by

$$
p(\bar{\omega}, x):=\left\{\begin{array}{ll}
w(\bar{\omega}, x)+\varepsilon & \text { if } x=x_{1}, \\
w(\bar{\omega}, x)-\varepsilon & \text { if } x=x_{2}, \\
w(\bar{\omega}, x) & \text { otherwise, }
\end{array} \quad q(\bar{\omega}, x):= \begin{cases}w(\bar{\omega}, x)-\varepsilon & \text { if } x=x_{1}, \\
w(\bar{\omega}, x)+\varepsilon & \text { if } x=x_{2}, \\
w(\bar{\omega}, x) & \text { otherwise. }\end{cases}\right.
$$

Since by assumption it must hold that $p \succ w$ and $q \succ w$, we deduce, thanks also to the convexity of the cone $\mathcal{C}$, that $0=(p-w)+(q-w) \in \mathcal{C}$. This contradicts the irreflexivity of the preference relation, through Proposition 1(i). 
Proof of Proposition 3. It follows from the definition of $\sigma$ that

$$
\sigma(\alpha p+(1-\alpha) q)=\alpha \sigma(p)+(1-\alpha) \sigma(q) \text { for all } \alpha \in(0,1) \text { and all } p, q \in \mathcal{H}
$$

and also that $\sigma^{-1}(z)=\omega$. This, together with the equivalent formulation of $\succ_{\sigma}$ as

$$
p \succ q \Leftrightarrow \sigma(p) \succ_{\sigma} \sigma(q)
$$

leads to the desired result.

Lemma 30. (i) Let $\mathcal{A}\left(\mathcal{H}_{z}\right)$ be given by Eq. (11). For any gamble $f \in \mathcal{L}\left(\Omega \times \mathcal{X}_{z}\right)$ it holds that

$$
f \in \mathcal{A}\left(\mathcal{H}_{z}\right) \Leftrightarrow(\forall \omega \in \Omega) \sum_{x \in \mathcal{X}_{z}} f(\omega, x)=0
$$

(ii) There is a one-to-one correspondence between elements of $\mathcal{A}\left(\mathcal{H}_{z}\right)$ and gambles in $\mathcal{L}(\Omega \times \mathcal{X})$.

Proof. (i) The direct implication is trivial. For the converse, we proceed with a constructive proof.

The case $f=0$ is trivial so we assume that $f \neq 0$. In order to normalise $f$ through $f:=f / \lambda$, we use $\lambda:=\sup _{\omega \in \Omega} \sum_{x \in \mathcal{X}_{z}}|f(\omega, x)|$, so that each of the previous sums is not greater than one. At this point for each $\omega \in \Omega$, we need to represent $f(\omega, \cdot)$ as the difference of two mass functions on $\mathcal{X}$, say $m_{\omega}^{+}-m_{\omega}^{-}$. We proceed as follows:

$$
\begin{aligned}
& m_{\omega}^{+}(x):= \begin{cases}0 & \text { if } x \neq z \text { and } f(\omega, x) \leq 0 \\
f(\omega, x) & \text { if } x \neq z \text { and } f(\omega, x)>0 \\
1-\sum_{x \in \mathcal{X}} m_{\omega}^{+}(x) & \text { if } x=z .\end{cases} \\
& m_{\omega}^{-}(x):= \begin{cases}0 & \text { if } x \neq z \text { and } f(\omega, x) \geq 0 \\
-f(\omega, x) & \text { if } x \neq z \text { and } f(\omega, x)<0 \\
1-\sum_{x \in \mathcal{X}} m_{\omega}^{-}(x) & \text { if } x=z .\end{cases}
\end{aligned}
$$

The difference $m_{\omega}^{+}-m_{\omega}^{-}$reproduces $f(\omega, x)$ for all $x \neq z$. In the case $x=z$, by assumption $f(\omega, z)=-\sum_{x \neq z} f(\omega, x)$, and since

$$
\begin{aligned}
-\sum_{x \neq z} f(\omega, x) & =\sum_{x \neq z: f(\omega, x)<0}-f(\omega, x)-\sum_{x \neq z: f(\omega, x)>0} f(\omega, x) \\
& =\left(1-\sum_{x \neq z: f(\omega, x)>0} f(\omega, x)\right)-\left(1-\sum_{x \neq z: f(\omega, x)<0}-f(\omega, x)\right),
\end{aligned}
$$

also $f(\omega, z)=m_{\omega}^{+}(z)-m_{\omega}^{-}(z)$. Since this holds for all $\omega \in \Omega, f$ can be represented as the difference of two horse lotteries.

Finally, it is enough to multiply $f$ by $\lambda$ to go back to the original, unnormalised, gamble, which is then an element of $\mathcal{A}\left(\mathcal{H}_{z}\right)$. 
(ii) To any $\lambda(p-q) \in \mathcal{A}\left(\mathcal{H}_{z}\right)$, we associate the gamble $f:=\lambda \psi(p-q)$. Let us show that such a map is injective. Assume that there are $\lambda_{1}\left(p_{1}-q_{1}\right)$ and $\lambda_{2}\left(p_{2}-q_{2}\right)$ that are both mapped to the same gamble $f$. This makes it trivial that $\lambda_{1}\left(p_{1}-q_{1}\right)(\omega, x)=$ $f(\omega, x)=\lambda_{2}\left(p_{2}-q_{2}\right)(\omega, x)$ for all $\omega \in \Omega$ and $x \neq z$. In the case of $x=z$, it is enough to write

$$
\begin{aligned}
\lambda_{1}\left(p_{1}-q_{1}\right)(\omega, z)= & -\lambda_{1} \sum_{x \in \mathcal{X}}\left(p_{1}-q_{1}\right)(\omega, x) \\
& =-\lambda_{2} \sum_{x \in \mathcal{X}}\left(p_{2}-q_{2}\right)(\omega, x)=\lambda_{2}\left(p_{2}-q_{2}\right)(\omega, z)
\end{aligned}
$$

for every $\omega \in \Omega$. As a consequence, $\lambda_{1}\left(p_{1}-q_{1}\right)=\lambda_{2}\left(p_{2}-q_{2}\right)$.

To prove surjectivity, if we take a gamble $f \in \mathcal{L}(\Omega \times \mathcal{X})$, we can always extend it to a gamble $g \in \mathcal{L}\left(\Omega \times \mathcal{X}_{z}\right)$ via

$$
g(x):= \begin{cases}f(x) & \text { if } x \neq z \\ -\sum_{x \in \mathcal{X}} f(x) & \text { if } x=z .\end{cases}
$$

Applying statement (i), we deduce that there are acts $p, q$ and $\lambda>0$ such that $g=\lambda(p-q)$, whence $f=\lambda \psi(p-q)$.

Remark 5 (Inverse of the projection operator). In some of the proofs we shall need to take the inverse of the projection operator $\psi$. Although $\psi$ is not injective in general, both its restrictions to $\mathcal{H}_{z}$ and $\mathcal{A}\left(\mathcal{H}_{z}\right)$ are. As a consequence, we shall denote by $\psi_{1}$ the restriction of the projection operator to $\mathcal{H}_{z}$, and then define its inverse

$$
\begin{aligned}
\psi_{1}^{-1}(f): \Omega \times \mathcal{X}_{z} & \rightarrow \mathbb{R} \\
(\omega, x) & \mapsto \begin{cases}f(\omega, x) & \text { if } x \neq z \\
1-\sum_{x \in \mathcal{X}} f(\omega, x) & \text { if } x=z .\end{cases}
\end{aligned}
$$

Similarly, we shall denote by $\psi_{2}$ the restriction of $\psi$ to $\mathcal{A}\left(\mathcal{H}_{z}\right)$, and define its inverse

$$
\begin{aligned}
\psi_{2}^{-1}(f): \Omega \times \mathcal{X}_{z} & \rightarrow \mathbb{R} \\
(\omega, x) & \mapsto \begin{cases}f(\omega, x) & \text { if } x \neq z \\
-\sum_{x \in \mathcal{X}} f(\omega, x) & \text { if } x=z . \diamond\end{cases}
\end{aligned}
$$

Proof of Theorem 4. Let us begin with the first statement. Let us show that $\mathcal{R}$ satisfies D1-D4:

D1. Consider $f \in \mathcal{L}^{+}(\Omega \times \mathcal{X})$ and let $\lambda:=\sup _{\omega \in \Omega} \sum_{x \in \mathcal{X}} f(\omega, x)$. Note that $\lambda>0$ otherwise $f$ would not be positive and moreover that $\lambda<+\infty$ because $f$ is bounded. Let $p \in \mathcal{H}_{z}$ be defined by

$$
p(\omega, x):= \begin{cases}\frac{f(\omega, x)}{\lambda} & \text { if } x \neq z, \\ 1-\sum_{x \neq z} \frac{f(\omega, x)}{\lambda} & \text { otherwise. }\end{cases}
$$

It follows that $\psi(\lambda(p-z))=f$ and since $p \neq z$ because $f$ is positive, then $p \succ z$ and hence $f \in \mathcal{R}$. 
D2. $\mathcal{C}$ does not contain the origin by Proposition 1 (ii) and hence $0 \notin \mathcal{R}$.

D3. This follows from the convexity of $\mathcal{C}$, again by Proposition 1(ii) and the linearity of $\psi$.

D4. This follows again from the convexity of $\mathcal{C}$ taking into consideration that $\psi$ is a linear operator.

The remaining part of the statement follows from the definition of $\mathcal{R}$.

Let us turn now towards the second statement. Define $\mathcal{C}:=\psi_{2}^{-1}(\mathcal{R})$. By Lemma 30, $\emptyset \neq \mathcal{C} \subseteq \mathcal{A}\left(\mathcal{H}_{z}\right) \backslash\{0\}$. To show that $\mathcal{C}$ is convex, it suffices to note that $\mathcal{R}$ is convex and that the operator $\psi_{2}^{-1}$ is linear, and thus preserves convexity.

Using Proposition 1(iii), we obtain that $\mathcal{R}$ induces the following coherent preference relation:

$$
p \succ q \Leftrightarrow \lambda(p-q) \in \mathcal{C} \Leftrightarrow \lambda \psi(p-q) \in \mathcal{R} .
$$

To show that $p \succ z$ for all $p \in \mathcal{H}_{z}, p \neq z$, it is then enough to consider that $\psi(p-z)$ is a positive gamble.

Proof of Proposition 5. If $p \triangleright q$, then it follows that $\psi(p-q) \in \mathcal{L}^{+}(\Omega \times \mathcal{X})$. By Theorem 4(i), $\psi(p-q) \in \mathcal{R}$, and as a consequence $p-q \in \mathcal{C}$. Applying now Proposition 1, we conclude that $p \succ q$.

Proof of Proposition 6. We begin with the direct implication. Assume first of all that $\left|\mathcal{X}_{z}\right| \geq 3$. Let $u$ be the uniform act defined by $u(\omega, x):=1 /\left|\mathcal{X}_{z}\right|$ for all $\omega \in \Omega$. Then fix any $x^{\prime} \neq z$ and $\varepsilon>0$ small enough so as to define the act $p$ given, for all $\omega \in \Omega$, by

$$
p(\omega, x):= \begin{cases}u(\omega, x)+\varepsilon & \text { if } x=x^{\prime} \\ u(\omega, x)-\varepsilon & \text { if } x=z \\ u(\omega, x) & \text { otherwise }\end{cases}
$$

It follows that $p \succ u$ since $p \triangleright u$. Given that also $u \succ z$, we can apply the Archimedean axiom A3 to obtain that there is a $\beta \in(0,1)$ such that:

$$
\beta p+(1-\beta) z \succ u \text {. }
$$

But $\beta p+(1-\beta) z \not u$ : it is enough to notice that for any $\omega \in \Omega$ and any $x \neq x^{\prime}, z$, it holds that $[\beta p+(1-\beta) z-u](\omega, x)=(\beta-1) /\left|\mathcal{X}_{z}\right|<0$.

Next, if $\left|\mathcal{X}_{z}\right|=2$ and $|\Omega| \geq 2$, then, with $x \neq z$ we can take $\omega_{1} \in \Omega$ and define horse lotteries $p, q$ by $p\left(\omega_{1}, x\right):=1, q\left(\omega_{1}, x\right):=0, p\left(\omega^{\prime}, x\right):=q\left(\omega^{\prime}, x\right):=\frac{1}{2}$ for any $\omega^{\prime} \neq \omega_{1}$. Then $p \triangleright q \triangleright z$, whence $p \succ q \succ z$. However, for any $\beta \in(0,1)$ it holds that

$$
\left(\forall \omega^{\prime} \neq \omega_{1}\right) \beta p\left(\omega^{\prime}, x\right)+(1-\beta) z\left(\omega^{\prime}, x\right)<q\left(\omega^{\prime}, x\right)
$$

whence $\beta p+(1-\beta) z \not q$ and as a consequence $\succ$ is not Archimedean.

Conversely, given $\Omega:=\{\omega\}, \mathcal{X}_{z}:=\{x, z\}$, then it holds that $p \triangleright q \Leftrightarrow p(\omega, x)>q(\omega, x)$, and then if $p \succ q \succ r$ there are $\alpha, \beta \in(0,1)$ such that $\alpha p(\omega, x)+(1-\alpha) r(\omega, x)>q(\omega, x)>$ $\beta p(\omega, x)+(1-\beta)>q(\omega, x)$, meaning that $\alpha p+(1-\alpha) r \succ q \succ \beta p+(1-\beta) r$, and as a consequence $\succ$ is Archimedean. 
Proof of Proposition 7. Let us begin by proving that wA3 $\Leftrightarrow \mathrm{A} 3^{\prime}$ :

$(\Rightarrow)$ Consider $p, q, r \in \mathcal{H}_{z}$ with $z \neq z$ such that $p \succ q \succ r$ and $p \not q$. By wA3, $\alpha p+$ $(1-\alpha) z \succ q$ for some $\alpha \in(0,1)$. And since by Proposition 1(iv) we have that $\alpha p+(1-\alpha) r \succ \alpha p+(1-\alpha) z$, we deduce that $\alpha p+(1-\alpha) r \succ q$.

$(\Leftarrow)$ This implication follows by a direct application of $\mathrm{A} 3^{\prime}$ to $p, q \in \mathcal{H}_{z}$ such that $p \succ$ $q, p \not q$, given that $q \succ z$ (note that it cannot be $q=z$ because this would imply that $p \triangleright q)$.

Assume now that $\succ$ satisfies A $3^{\prime}$ and take $p \succ q \succ r, q \not r$; we have by A2 that $\alpha q+(1-$ $\alpha) p \succ \alpha r+(1-\alpha) p \succ \alpha r+(1-\alpha) q$ for any $\alpha \in(0,1)$. We apply A3' to the latter chain of preferences; note that this requires that $\alpha q+(1-\alpha) p \not \alpha r+(1-\alpha) p$, which is true since $q \not r$. As a consequence, there is $\gamma \in(0,1)$ such that $\gamma(\alpha q+(1-\alpha) p)+(1-\gamma)(\alpha r+(1-\alpha) q) \succ$ $\alpha r+(1-\alpha) p$, or, equivalently, that $\alpha \gamma(q-r)-(1-\alpha)(1-\gamma)(p-q) \in \mathcal{C}$, where $\mathcal{C}$ is the cone associated with $\succ$. Normalising we find

$$
\beta=\frac{(1-\alpha)(1-\gamma)}{\alpha \gamma+(1-\alpha)(1-\gamma)} \in(0,1)
$$

such that $\beta q+(1-\beta) q=q \succ \beta p+(1-\beta) r$.

Next we give a lemma that considers a special translation of a coherent set of desirable gambles. Its proof, needed for Theorem 8 , is a reformulation of ideas put forward by Galaabaatar and Karni (2013, Lem. 1), on top of which we establish the connection between wA3 and D0.

Lemma 31. Let $\mathcal{R}$ be the coherent set of desirable gambles in $\mathcal{L}(\Omega \times \mathcal{X})$ arising from a coherent preference relation $\succ$ on $\mathcal{H}_{z} \times \mathcal{H}_{z}$ through (13). Denote by $u$ the uniform act in $\mathcal{H}_{z}$ defined by $u(\omega, x):=1 /\left|\mathcal{X}_{z}\right|$ for all $\omega \in \Omega, x \in \mathcal{X}_{z}$. Consider the translation map $\tau: \mathcal{L} \rightarrow \mathcal{L}$ given by $\tau(f)=\psi(u)+f$, the following translated sets:

$$
\begin{aligned}
\tau(\mathcal{R}) & :=\{g \in \mathcal{L}(\Omega \times \mathcal{X}): g=\psi(u)+f, f \in \mathcal{R}\}, \\
\tau\left(\mathcal{L}^{+}(\Omega \times \mathcal{X})\right) & :=\{g \in \mathcal{L}(\Omega \times \mathcal{X}): g \geqslant \psi(u)\},
\end{aligned}
$$

and the following version of D0 adapted to $\tau(\mathcal{R})$ :

$\mathrm{D}^{\prime} . g \in \tau(\mathcal{R}) \backslash \tau\left(\mathcal{L}^{+}(\Omega \times \mathcal{X})\right) \Rightarrow(\exists \delta>0) g-\delta \in \tau(\mathcal{R})$.

Then it follows that:

(i) D0 and $\mathrm{D} 0$ ' are equivalent conditions.

(ii) $\tau(\mathcal{R})=\{\psi(u)+\lambda \psi(p-u): \lambda>0, p \succ u\}$.

Proof. Note first that if $g=\psi(u)+f, g \in \tau(\mathcal{R})$, then $f \in \mathcal{R}$ : in fact if $g \in \tau(\mathcal{R})$, then $g=\psi(u)+h$ for some $h \in \mathcal{R}$, whence $f=h$. 
(i) Let us show that $\mathrm{D} 0$ and $\mathrm{D} 0^{\prime}$ are equivalent conditions.

Consider $g \in \tau(\mathcal{R}) \backslash \tau\left(\mathcal{L}^{+}(\Omega \times \mathcal{X})\right)$; then $g=\psi(u)+f, f \in \mathcal{R} \backslash \mathcal{L}^{+}(\Omega \times \mathcal{X})$. Applying D0, there is $\delta>0$ such that $f-\delta \in \mathcal{R}$, whence $\psi(u)+f-\delta=g-\delta \in \tau(\mathcal{R})$.

Conversely, if $f \in \mathcal{R} \backslash \mathcal{L}^{+}(\Omega \times \mathcal{X})$, then $\psi(u)+f \in \tau(\mathcal{R}) \backslash \tau\left(\mathcal{L}^{+}(\Omega \times \mathcal{X})\right)$; applying D0' we get that there is $\delta>0$ such that $\psi(u)+f-\delta \in \tau(\mathcal{R})$, whence $f-\delta \in \mathcal{R}$.

(ii) It is trivial that $\{\psi(u)+\lambda \psi(p-u): \lambda>0, p \succ u\} \subseteq \tau(\mathcal{R})$, so we concentrate on the converse inclusion.

Consider $\psi(u)+f$. Then there are $\lambda>0$ and $p_{1} \succ p_{2}$ such that $f=\lambda \psi\left(p_{1}-\right.$ $p_{2}$ ). Remember that $\left|\mathcal{X}_{z}\right| \geq 2$ by assumption (see Remark 3); whence, if we take $\mu \in\left(0, \frac{1}{\lambda\left|\mathcal{X}_{z}\right|}\right)$, we obtain that $\left[u+\mu \lambda\left(p_{1}-p_{2}\right)\right](\omega, x) \in[0,1]$ for all $(\omega, x) \in \Omega \times$ $\mathcal{X}_{z}$. Since in addition $\sum_{x \in \mathcal{X}_{z}}\left[p_{1}-p_{2}\right](\omega, x)=0$ for all $\omega \in \Omega$, we have also that $\sum_{x \in \mathcal{X}_{z}}\left[u+\mu \lambda\left(p_{1}-p_{2}\right)\right](\omega, x)=\sum_{x \in \mathcal{X}_{z}} u(\omega, x)=1$ for all $\omega \in \Omega$. We deduce that $p:=u+\mu \lambda\left(p_{1}-p_{2}\right) \in \mathcal{H}_{z}$.

Given that $p-u=\mu \lambda\left(p_{1}-p_{2}\right)$ and that $p_{1} \succ p_{2}$, we obtain from Proposition 1(iv) that $p \succ u$. And since $\psi(u)+f=\psi(u)+\frac{1}{\mu} \psi(p-u)$, we deduce that $\psi(u)+f \in$ $\{\psi(u)+\lambda \psi(p-u): \lambda>0, p \succ u\}$.

Proof of Theorem 8. (i) Coherence follows from Theorem 4. In order to prove that $\mathcal{R}$ is a set of strictly desirable gambles, it is enough to show that D0' holds, thanks to Lemma 31.

Consider $g \in \tau(\mathcal{R}) \backslash \tau\left(\mathcal{L}^{+}(\Omega \times \mathcal{X})\right)$. Then, according to Lemma 31, $g=\psi(u)+f$, with $f:=\lambda \psi(p-u) \in \mathcal{R}, \lambda>0$, and for some $p \in \mathcal{H}_{z}$ such that $p \succ u, p \not u$.

Since $u \succ z$, it follows from wA3 that there is some $\beta \in(0,1)$ such that $\beta p+(1-\beta) z \succ$ $u$, whence

$$
\psi(\beta(p-u)-(1-\beta)(u-z))=\frac{\beta f}{\lambda}-(1-\beta) \psi(u) \in \mathcal{R} .
$$

Since $\mathcal{R}$ is a cone, this means that $f-\frac{\lambda(1-\beta)}{\beta} \psi(u) \in \mathcal{R}$ for some $\beta \in(0,1)$, or, in other words, that $f-\delta \in \mathcal{R}$, with $\delta:=\frac{\lambda(1-\beta)}{\beta\left|\mathcal{X}_{z}\right|}>0$. We deduce that $g-\delta=\psi(u)+f-\delta \in$ $\tau(\mathcal{R})$, whence $\mathrm{D}^{\prime}$ holds and as a consequence $\mathcal{R}$ is a coherent set of strictly desirable gambles.

(ii) Let $\mathcal{C}:=\psi_{2}^{-1}(\mathcal{R})$. From the proof of Theorem $4, \mathcal{R}$ induces the following coherent preference relation:

$$
p \succ q \Leftrightarrow \lambda(p-q) \in \mathcal{C} \Leftrightarrow \lambda \psi(p-q) \in \mathcal{R} .
$$

We are left to show that if $\mathcal{R}$ is strictly desirable, then $\succ$ is weakly Archimedean. Let us consider $p \succ q, p \not q$. By D0, there is $\delta>0$ such that $\psi(p-q)-\delta \in \mathcal{R}$. Choose $\beta \in(0,1)$ so that $\psi((1-\beta) p) \leq \delta$. Then $\psi(\beta p-q) \geq \psi(p-q)-\delta$, whence $\psi(\beta p-q) \in \mathcal{R}$, which implies that $\beta p+(1-\beta) z \succ q$.

Proof of Theorem 9. (i) Let us consider the direct implication. If $p \triangleright q$, then $p \succ q$ holds as a consequence of Proposition 5 and the fact that $z$ is the worst outcome for 
$\succ$. Furthermore, if $r \succ s$, then using Definition 20, and recalling Remark 5, we obtain that $\psi_{1}^{-1}(r) \succ \psi_{1}^{-1}(s)$, which is equivalent to $\psi_{2}^{-1}(r-s) \in \mathcal{C}$, where $\mathcal{C}$ is the cone induced by $\succ$. As a consequence, $p-q=\psi_{2}^{-1}(r-s) \in \mathcal{C}$, whence $p \succ q$.

For the converse implication, it is enough to note that $p \triangleright z$ for all $p \neq z$, so that $p \succ z$; and that, on the other hand, $r \succ s$ and $\psi\left(\psi_{1}^{-1}(r)-\psi_{1}^{-1}(s)\right)=r-s$ imply that $\psi_{1}^{-1}(r) \succ \psi_{1}^{-1}(s)$.

(ii) Remember that any extension has a corresponding convex cone $\mathcal{C}$ (which is nonempty because of the worst outcome). We know by the first point in the theorem that $\psi_{2}^{-1}(r-s) \in \mathcal{C}$ if $r \succ s$ and moreover that $t-z \in \mathcal{C} \cup\{0\}$ for any $t \in \mathcal{H}_{z}$. Given that $\mathcal{C}$ is convex, then also $\lambda_{1} \psi_{2}^{-1}(r-s)+\lambda_{2}(t-z) \in \mathcal{C}$ for all $\lambda_{1}, \lambda_{2}>0$. In other words, if there are $p, q \in \mathcal{H}_{z}$ and $\lambda_{3}>0$ such that $\lambda_{1} \psi_{2}^{-1}(r-s)+\lambda_{2}(t-z)=\lambda_{3}(p-q)$, then $p \succ q$. Equivalently, $p \succ q$ if $\psi(p-q) \geq \lambda(r-s), r, s \in \mathcal{H}, r \succ s, \lambda>0$, where the inequality is obtained by letting $\lambda:=\lambda_{1} / \lambda_{3}$ and observing that $\psi\left(\lambda_{2}(t-z)\right)=\lambda_{2} \psi(t)$ spans the entire set $\{f \geq 0\}$ when $t \in \mathcal{H}_{z}$. This allows us to reformulate (14) in an equivalent way as follows, for all $p, q \in \mathcal{H}_{z}$ :

$$
p \succ q \text { if } p \triangleright q \text { or } \psi(p-q) \geq \lambda(r-s) \text { for some } r, s \in \mathcal{H}, r \succ s, \lambda>0 \text {. }
$$

At this point we consider $\mathcal{C}^{\prime}:=\{\lambda(r-s): r, s \in \mathcal{H}, r \succ s, \lambda>0\}$. Assume for the moment that $\mathcal{C}^{\prime}$ is non-empty. Then $\mathcal{C}^{\prime}$ is a convex cone that does not include the origin. If we regard $\mathcal{C}^{\prime}$ as a set of gambles in $\mathcal{L}(\Omega \times \mathcal{X})$, then we see that it is convex and does not contain zero; but it is not coherent because it does not contain $\mathcal{L}^{+}$. We can therefore take the smallest convex cone that includes both $\mathcal{C}^{\prime}$ and $\mathcal{L}^{+}$:

$$
\mathcal{R}:=\{f \geq \lambda(r-s): r, s \in \mathcal{H}, r \succ s, \lambda>0)\} \cup \mathcal{L}^{+} .
$$

$\mathcal{R}$ is the natural extension of $\mathcal{C}^{\prime}$, that is, the least-committal extension of $\mathcal{C}^{\prime}$ to a coherent set. Observe that if $\mathcal{C}^{\prime}=\emptyset$, then $\mathcal{R}=\mathcal{L}^{+}$, which is the vacuous set of gambles; therefore $\mathcal{R}$ represents the correct extension also in this special case.

Given that $\mathcal{R}$ is a coherent set of gambles on $\Omega \times \mathcal{X}$, we can use Theorem 4 to create a new coherent preference relation $\succ$ on $\mathcal{H}_{z} \times \mathcal{H}_{z}$, with corresponding cone $\mathcal{C}:=\psi^{-1}(\mathcal{R})$, for which $z$ is the worst outcome. Recalling that $p \succ q \Leftrightarrow \lambda \psi(p-q) \in \mathcal{R}$ for some $\lambda>0$, it follows that the new relation $\succ$ contains all the preferences expressed by (29) and only them: either $\lambda \psi(p-q) \in \mathcal{L}^{+}$, in which case $p \triangleright q$, or $\lambda \psi(p-q) \geq \lambda^{\prime}(r-s)$ for some $\lambda^{\prime}>0, r, s \in \mathcal{H}, r \succ s$. As a consequence it is the minimal extension of $\succ$ on $\mathcal{H} \times \mathcal{H}$ to the worst outcome, because any extension has to express at least those preferences.

(iii) Assume first of all that $\mathcal{C} \neq \emptyset$, and let us prove that $\underline{P}(f)=0$ for every $f \in \mathcal{C}$. This will imply that $\mathcal{R}$ is not a coherent set of strictly desirable gambles, because $\mathcal{C} \subseteq \mathcal{R} \backslash \mathcal{L}^{+}$, and by Eq. (7) we should have $\underline{P}(f)>0$ for every $f \in \mathcal{R} \backslash \mathcal{L}^{+}$.

Since $\mathcal{C} \subseteq \mathcal{R}$, it follows from Eq. (5) that $\underline{P}(f) \geq 0$ for every $f \in \mathcal{C}$. On the other hand, if $\underline{P}(f)>0$ for some $f \in \mathcal{C}$, there should be some $\varepsilon>0$ such that $f-\varepsilon \in \mathcal{R}$; but then we would have that for any $\omega \in \mathcal{X}, \sum_{x \in \mathcal{X}}(f-\varepsilon)(\omega, x)=-\varepsilon|\mathcal{X}|<0$, while 
by construction any gamble $g \in \mathcal{R}$ satisfies $\sum_{x \in \mathcal{X}} g(\omega, x) \geq 0$. Thus, $\underline{P}(f)=0$ and as a consequence, $\mathcal{R}$ is not a set of strictly desirable gambles.

The converse implication, where $\mathcal{C}=\emptyset$, follows from the fact that in this case $\mathcal{R}=\mathcal{L}^{+}$, which is a set of strictly desirable gambles.

Proof of Corollary 10. Denote by $\underline{P}_{1}$ the coherent lower prevision associated with $\mathcal{R}_{1}$ and by $\underline{P}$ the lower prevision induced by $\mathcal{R}$. Consider $f \in \mathcal{C}$. We know by point (iii) in Theorem 9 that $\underline{P}(f)=0$. In addition to $\mathcal{R}_{1}$ including $\mathcal{R}$, consider that it is a set of strictly desirable gambles and that $f \notin \mathcal{L}^{+}$, whence $\underline{P}_{1}(f)>0$. Written in other words:

$$
(\forall f \in \mathcal{C}) \underline{P}(f)=0<\underline{P}_{1}(f) .
$$

It follows that $\mathcal{M}\left(\underline{P}_{1}\right) \subsetneq \mathcal{M}(\underline{P})$ and that any linear prevision $P_{1} \geq \underline{P}_{1}$ satisfies $P_{1}(f)>0$ for every $f \in \mathcal{C}$. Fix now $f \in \mathcal{C}$, consider $P_{1} \in \mathcal{M}\left(\underline{P}_{1}\right)$ and $P \in \mathcal{M}(\underline{P})$ such that $P_{1}(f)=\underline{P}_{1}(f)$ and $P(f)=\underline{P}(f)$. Take $P_{2}:=\frac{1}{2} P_{1}+\frac{1}{2} P \in \mathcal{M}(\underline{P})$, and let $\mathcal{M}_{2}$ be the convex hull of $\mathcal{M}\left(\underline{P}_{1}\right) \cup\left\{P_{2}\right\}$. Its lower envelope $\underline{P}_{2}$ is a lower prevision that lies between $\underline{P}$ and $\underline{P}_{1}$ and satisfies in particular $\underline{P}_{2}(f)=\underline{P}_{1}(f) / 2$. As a consequence, its associated set of strictly desirable gambles $\mathcal{R}_{2}$ is a proper subset of $\mathcal{R}_{1}$ and moreover it properly includes $\mathcal{R}$ (because $\underline{P}_{2}(g)>0$ for all $g \in \mathcal{C}$ by construction while $\left.\underline{P}(g)=0\right)$.

Proof of Theorem 11. As usual, given a coherent preference relation $\succ$, we map it into the set of gambles defined by $\mathcal{R}:=\psi(\mathcal{C})$, where $\mathcal{C}:=\{\lambda(p-q): p \succ q, \lambda>0\}$. The set $\mathcal{R}$ is coherent as a consequence of Theorem 4. That the map is injective follows considered that $\psi_{2}$ (the restriction of $\psi$ to scaled differences of acts) is invertible. That it is surjective follows from Theorem 4. The equivalence of the weak Archimedean condition and strict desirability follows from Theorem 8. Finally, the relation and the set of strictly desirable gambles give rise to the same representation because $\mathcal{C}$ and $\mathcal{R}$ induce the same set of linear previsions.

Proof of Proposition 12. It follows from the work by Miranda et al. (2012, Prop. 29) that $\hat{\mathcal{R}}$ is the smallest conglomerable and coherent set of gambles that includes $\mathcal{R}_{\Omega}$ and $\mathcal{R} \mid\{\omega\}$ (for all $\omega \in \Omega$ ). It includes $\mathcal{R} \mid \Omega$ by construction. We are left to show that $\hat{\mathcal{R}}$ induces both $\mathcal{R}_{\Omega}$ and $\mathcal{R}_{\mathcal{X}}$

We begin by proving that the $\Omega$-marginal of $\hat{\mathcal{R}}$ is $\mathcal{R}_{\Omega}$. Consider an $\Omega$-measurable gamble $f \in \hat{\mathcal{R}}$. Then there are $g \in \mathcal{R}_{\Omega}$ and $h \in \mathcal{R} \mid \Omega$ such that $f \geq g+h$. For any $\omega \in \Omega$, it holds that

$$
0 \leq \max _{x \in \mathcal{X}} h(\omega, x) \leq \max _{x \in \mathcal{X}}(f(\omega, x)-g(\omega, x))=f^{\prime}(\omega)-g^{\prime}(\omega),
$$

where in last equality we are using that both $f, g$ are $\Omega$-measurable, and are denoting by $f^{\prime}, g^{\prime}$ their equivalent representations as gambles on $\Omega$. Thus, $f \geq g$, and since $\mathcal{R}_{\Omega}$ is a coherent set of gambles we conclude that also $f \in \mathcal{R}_{\Omega}$. The converse inclusion follows from Eq. (18).

Fix next $\omega \in \Omega$. If $f I_{\{\omega\}} \in \hat{\mathcal{R}}$, then there are $g \in \mathcal{R}_{\Omega}$ and $h \in \mathcal{R} \mid \Omega$ such that $f I_{\{\omega\}} \geq$ $g+h$. For any $\omega^{\prime} \neq \omega$, it holds that

$$
(\forall x \in \mathcal{X}) 0 \geq g\left(\omega^{\prime}, x\right)+h\left(\omega^{\prime}, x\right)
$$


whence it must hold that $g\left(\omega^{\prime}, x\right) \leq \min _{x \in \mathcal{X}}-h\left(\omega^{\prime}, x\right) \leq 0$ for every $x \in \mathcal{X}$, taking into account that $g$ is $\Omega$-measurable. Thus, if we consider instead $g^{\prime}:=g I_{\{\omega\}} \geq g \in \mathcal{R}_{\Omega}$ and $h^{\prime}:=h I_{\{\omega\}} \in \mathcal{R} \mid \Omega$, we also have that $f I_{\{\omega\}} \geq g^{\prime}+h^{\prime}$. Taking into account that $g^{\prime} \in \mathcal{R}_{\Omega}$, it should be $g^{\prime}(\omega, x)=g^{\prime}\left(\omega, x^{\prime}\right) \geq 0$ for every $x, x^{\prime} \in \mathcal{X}$, whence $f I_{\{\omega\}} \geq h I_{\{\omega\}}$ and thus $f \in \mathcal{R}_{\mathcal{X}}$. Again, the converse inclusion follows from Eq. (18).

Proof of Proposition 13. This follows from the definition of natural extension of a set of desirable gambles by Walley (1991, sec. 3.7), taking also into account that both $\mathcal{R}|\mathcal{X}, \mathcal{R}| \Omega$ are convex cones of gambles that include all non-negative gambles.

Proof of Proposition 14. The result follows from Definition 26, taking into account that this concatenation is a coherent lower prevision, by Walley (1991, sec. 6.7).

Proof of Proposition 15. We prove that (i) $\Leftrightarrow($ ii), that (ii) $\Leftrightarrow$ (iii) and that $(\mathrm{i}) \Leftrightarrow($ iv $)$.

$(i) \Leftrightarrow($ ii) This has been proven by Miranda and Zaffalon (2010, Prop. 6).

(ii) $\Rightarrow$ (iii) Consider gambles $f, g \notin \mathcal{R}$. We have that $\varepsilon / 2-f, \varepsilon / 2-g \in \mathcal{R}$ for all $\varepsilon>0$; whence $\varepsilon-(f+g) \in \mathcal{R}$ for all $\varepsilon>0$. By D2 and D4, it follows that $f+g-\varepsilon \notin \mathcal{R}$ for all $\varepsilon>0$.

$($ iii $) \Rightarrow($ ii $)$ Assume ex-absurdo that there is a gamble $h$ and $\varepsilon>0$ such that $h, \varepsilon-h \notin \mathcal{R}$. We obtain that $h+\varepsilon-h+\delta=\varepsilon+\delta \notin \mathcal{R}$ for all $\delta>0$, which contradicts D1.

$(i) \Rightarrow(i v)$ Note that

$$
\begin{array}{r}
\alpha \mu_{1}+(1-\alpha) \mu_{2} \succ \mu_{1} I_{A}+\mu_{2} I_{A^{c}} \Leftrightarrow \\
(1-\alpha)\left(\mu_{2}-\mu_{1}\right) I_{A}+\alpha\left(\mu_{1}-\mu_{2}\right) I_{A^{c}} \in \mathcal{R},
\end{array}
$$

and similarly

$$
\begin{array}{r}
\mu_{1} I_{A}+\mu_{2} I_{A^{c}} \succ \alpha^{\prime} \mu_{1}+\left(1-\alpha^{\prime}\right) \mu_{2} \Leftrightarrow \\
\left(1-\alpha^{\prime}\right)\left(\mu_{1}-\mu_{2}\right) I_{A}+\alpha^{\prime}\left(\mu_{2}-\mu_{1}\right) I_{A^{c}} \in \mathcal{R} .
\end{array}
$$

Now, if $P$ is a linear prevision,

$$
P\left((1-\alpha)\left(\mu_{2}-\mu_{1}\right) I_{A}+\alpha\left(\mu_{1}-\mu_{2}\right) I_{A^{c}}\right)=\left(\mu_{2}-\mu_{1}\right)(P(A)-\alpha) .
$$

Hence, there are two possibilities: either $P(A)<\alpha$, and then $P\left((1-\alpha)\left(\mu_{2}-\mu_{1}\right) I_{A}+\right.$ $\left.\alpha\left(\mu_{1}-\mu_{2}\right) I_{A^{c}}\right)>0$ and as a consequence $(1-\alpha)\left(\mu_{2}-\mu_{1}\right) I_{A}+\alpha\left(\mu_{1}-\mu_{2}\right) I_{A^{c}} \in \mathcal{R}$; or $P(A) \geq \alpha$, whence for every $\alpha^{\prime}<\alpha$ it holds that

$$
P\left(\left(1-\alpha^{\prime}\right)\left(\mu_{1}-\mu_{2}\right) I_{A}+\alpha^{\prime}\left(\mu_{2}-\mu_{1}\right) I_{A^{c}}\right)=\left(\mu_{1}-\mu_{2}\right)\left(P(A)-\alpha^{\prime}\right)>0,
$$

and as a consequence $\left(1-\alpha^{\prime}\right)\left(\mu_{1}-\mu_{2}\right) I_{A}+\alpha^{\prime}\left(\mu_{2}-\mu_{1}\right) I_{A^{c}} \in \mathcal{R}$. 
$(i v) \Rightarrow(i)$ If $\underline{P}$ is not a linear prevision, then its restriction to events cannot be additive, so there is some event $A$ such that $\underline{P}(A)<\bar{P}(A)$. Then if we take $\mu_{1}=1, \mu_{2}=0$ and $\alpha \in(\underline{P}(A), \bar{P}(A))$,

$$
\alpha \mu_{1}+(1-\alpha) \mu_{2}-\left(\mu_{1} I_{A}+\mu_{2} I_{A^{c}}\right)=\alpha-I_{A} \notin \mathcal{R} \text { because } \bar{P}(A)>\alpha,
$$

and similarly given $\alpha^{\prime} \in(\underline{P}(A), \alpha)$,

$$
\mu_{1} I_{A}+\mu_{2} I_{A^{c}}-\left(\alpha^{\prime} \mu_{1}+\left(1-\alpha^{\prime}\right) \mu_{2}\right)=I_{A}-\alpha^{\prime} \notin \mathcal{R} \text { because } \underline{P}(A)<\alpha^{\prime} .
$$

This is a contradiction.

Proof of Proposition 17. Let us begin by establishing the direct implication. Assume ex-absurdo that $\underline{P}$ is not dominated by the strong product $\underline{P}_{\Omega}^{\prime} \otimes \underline{P}_{\mathcal{X}}^{\prime}$. Then there are some $P_{\Omega}^{\prime} \in \mathcal{M}\left(\underline{P}_{\Omega}^{\prime}\right)$ and $P_{\mathcal{X}}^{\prime} \in \mathcal{M}\left(\underline{P}_{\mathcal{X}}^{\prime}\right)$ such that $P_{\Omega}^{\prime} \times P_{\mathcal{X}}^{\prime} \notin \mathcal{M}(\underline{P})$. Thus, there is some gamble $f$ in $\mathcal{L}(\Omega \times \mathcal{X})$ such that $\underline{P}(f)>\left(P_{\Omega}^{\prime} \times P_{\mathcal{X}}^{\prime}\right)(f)$. Furthermore,

$$
\left(P_{\Omega}^{\prime} \times P_{\mathcal{X}}^{\prime}\right)(f)=P_{\Omega}^{\prime}\left(P_{\mathcal{X}}(f \mid \Omega)\right) \geq \underline{P}_{\Omega}\left(P_{\mathcal{X}}(f \mid \Omega)\right)
$$

and this is a contradiction with Eq. (22).

Conversely, let us prove first of all that the strong product $\underline{P}_{\Omega}^{\prime} \otimes \underline{P}_{\mathcal{X}}^{\prime}$ satisfies Eq. (22). Consider $P_{\mathcal{X}}^{\prime} \geq \underline{P}_{\mathcal{X}}^{\prime}$ and a gamble $f \in \mathcal{L}(\Omega \times \mathcal{X})$. Given the gamble $P_{\mathcal{X}}(f \mid \Omega)$ on $\Omega$, there is some $P_{\Omega}^{\prime} \geq \underline{P}_{\Omega}^{\prime}$ such that

$$
P_{\Omega}^{\prime}\left(P_{\mathcal{X}}(f \mid \Omega)\right)=\underline{P}_{\Omega}^{\prime}\left(P_{\mathcal{X}}(f \mid \Omega)\right),
$$

whence $\underline{P}_{\Omega}^{\prime} \otimes \underline{P}_{\mathcal{X}}^{\prime}(f) \leq \underline{P}_{\Omega}^{\prime}\left(P_{\mathcal{X}}(f \mid \Omega)\right)$, and as a consequence (22) holds. Now, if $\underline{P} \leq \underline{P}_{\Omega}^{\prime} \otimes$ $\underline{P}_{\mathcal{X}}^{\prime}$, given $P_{\mathcal{X}}^{\prime} \geq \underline{P}_{\mathcal{X}}^{\prime}$ and a gamble $f \in \mathcal{L}(\Omega \times \mathcal{X})$, then $\underline{P}(f) \leq \underline{P}_{\Omega}^{\prime} \otimes \underline{P}_{\mathcal{X}}^{\prime}(f) \leq \underline{P}_{\Omega}^{\prime}\left(P_{\mathcal{X}}(f \mid \Omega)\right)$. Hence, $\underline{P}$ also satisfies (22).

Our next result is used in the proof of Lemma 18. It extends a result by de Cooman et al. (2011, Prop. 25).

Lemma 32. Let $\underline{P}$ be a coherent lower prevision on $\mathcal{L}(\Omega \times \mathcal{X})$ with marginals $\underline{P}_{\Omega}^{\prime}, \underline{P}_{\mathcal{X}}^{\prime}$. If $\underline{P}_{\mathcal{X}}^{\prime}=: P_{\mathcal{X}}^{\prime}$ is a linear prevision, then the only independent product of $\underline{P}_{\Omega}^{\prime}, P_{\mathcal{X}}^{\prime}$ is the strong product $\underline{P}_{\Omega}^{\prime} \otimes P_{\mathcal{X}}^{\prime}$, which coincides moreover with $\underline{P}_{\Omega}^{\prime}\left(P_{\mathcal{X}}(\cdot \mid \Omega)\right)$.

Proof. From a result by Miranda and Zaffalon (2015, Prop. 7), when $\underline{P}_{\mathcal{X}}^{\prime}$ is a linear prevision, then $\underline{P}$ is an independent product if and only if it dominates $\underline{P}_{\Omega}^{\prime}\left(P_{\mathcal{X}}(\cdot \mid \Omega)\right)$. Let us show that we must actually have the equality.

Given a gamble $f$, it holds that

$$
\begin{aligned}
& \underline{P}\left(f-P_{\mathcal{X}}(f \mid \Omega)\right) \geq \underline{P}_{\Omega}^{\prime}\left(P_{\mathcal{X}}\left(f-P_{\mathcal{X}}(f \mid \Omega)\right) \mid \Omega\right)=0 \text { and } \\
& \underline{P}\left(-f-P_{\mathcal{X}}(-f \mid \Omega)\right) \geq \underline{P}_{\Omega}^{\prime}\left(P_{\mathcal{X}}\left(-f-P_{\mathcal{X}}(-f \mid \Omega)\right) \mid \Omega\right)=0,
\end{aligned}
$$

whence

$$
\begin{aligned}
0 & =\underline{P}(0)=\underline{P}\left(\left(f-P_{\mathcal{X}}(f \mid \Omega)\right)+\left(-f-P_{\mathcal{X}}(-f \mid \Omega)\right)\right) \\
& \geq \underline{P}\left(f-P_{\mathcal{X}}(f \mid \Omega)\right)+\underline{P}\left(-f-P_{\mathcal{X}}(-f \mid \Omega)\right) \geq 0,
\end{aligned}
$$


and as a consequence $\underline{P}\left(f-P_{\mathcal{X}}(f \mid \Omega)\right)=0$ for every $f$. From this it follows that $\bar{P}(f-$ $\left.P_{\mathcal{X}}(f \mid \Omega)\right)=-\underline{P}\left(-\left(f-P_{\mathcal{X}}(f \mid \Omega)\right)\right)=-\underline{P}\left(-f-P_{\mathcal{X}}(-f \mid \Omega)\right)=0$ for every $f \in \mathcal{L}(\Omega \times \mathcal{X})$. Now, it is a consequence of a result by Walley (1991, Thm. 2.6.1(e)) that

$$
\underline{P}(f)=\underline{P}\left(\left(f-P_{\mathcal{X}}(f \mid \Omega)\right)+P_{\mathcal{X}}(f \mid \Omega)\right) \leq \bar{P}\left(f-P_{\mathcal{X}}(f \mid \Omega)\right)+\underline{P}\left(P_{\mathcal{X}}(f \mid \Omega)\right)=\underline{P}_{\Omega}^{\prime}\left(P_{\mathcal{X}}(f \mid \Omega)\right),
$$

and since we have the double inequality we obtain the equality. In particular the strong product, which is an independent product, must also coincide with $\underline{P}_{\Omega}^{\prime}\left(P_{\mathcal{X}}(\cdot \mid \Omega)\right)$.

Proof of Lemma 18. Let us first show that STP is equivalent to the following:

$$
(\forall g, f \in \mathcal{L}) \underline{P}(g-f) \geq \inf _{\omega \in \Omega} \underline{P}\left(g-f^{\omega}\right) .
$$

To show the direct implication, let $g^{\prime}:=g-\inf _{\omega \in \Omega} \underline{P}\left(g-f^{\omega}\right)$. Considered that $\inf _{\omega \in \Omega} \underline{P}\left(g^{\prime}-\right.$ $\left.f^{\omega}\right)=0$, we obtain by STP that $\underline{P}\left(g^{\prime}-f\right) \geq 0$, whence $\underline{P}(g-f) \geq \inf _{\omega \in \Omega} \underline{P}\left(g-f^{\omega}\right)$. For the converse implication, it is enough to consider that $\underline{P}(g-f) \geq \inf _{\omega \in \Omega} \underline{P}\left(g-f^{\omega}\right) \geq 0$.

Now, in the particular case that $\underline{P}$ is a linear prevision $P$ (with marginals $P_{\Omega}^{\prime}, P_{\mathcal{X}}^{\prime}$ ), then Eq. (30) is in turn equivalent to $P(-f) \geq \inf _{\omega \in \Omega} P\left(-f^{\omega}\right)=\inf _{\omega \in \Omega} P(-f \mid\{\omega\})$, where $P(\cdot \mid\{\omega\})$ is derived from $P_{\mathcal{X}}^{\prime}$ by Eq. (21). This is also equivalent to $P(f) \geq \inf _{\omega \in \Omega} P_{\mathcal{X}}(f \mid\{\omega\})$ because the former inequality has to hold for all gambles $f$.

If $P$ is an independent product of its marginals, then it is in particular coherent with $P_{\mathcal{X}}(\cdot \mid \Omega)$ (see Miranda \& Zaffalon, 2015, Def. 4), and applying a result by Walley (1991, Thm. 6.5.7), this is equivalent to $P(f) \geq \inf _{\omega \in \Omega} P_{\mathcal{X}}(f \mid\{\omega\})$. Conversely, if it is coherent with $P_{\mathcal{X}}(\cdot \mid \Omega)$, then applying a theorem by Walley (1991, Thm. 6.7.3) it must hold that $P=P_{\Omega}^{\prime}\left(P_{\mathcal{X}}(\cdot \mid \Omega)\right)$, and then, by Lemma $32, P$ is an independent product.

Let us show now that STP is preserved by taking lower envelopes. Let $\mathcal{M}$ be a family of linear previsions satisfying STP, and take $\underline{P}:=\inf \mathcal{M}$. Consider gambles $f, g \in \mathcal{L}(\Omega \times \mathcal{X})$. Then for any $\varepsilon>0$ there is some $P \in \mathcal{M}$ such that

$$
\begin{aligned}
\underline{P}\left(g-f-\inf _{\omega \in \Omega} \underline{P}\left(g-f^{\omega}\right)\right) & \geq P\left(g-f-\inf _{\omega \in \Omega} \underline{P}\left(g-f^{\omega}\right)\right)-\varepsilon \\
& \geq P\left(g-f-\inf _{\omega \in \Omega} P\left(g-f^{\omega}\right)\right)-\varepsilon \geq-\varepsilon,
\end{aligned}
$$

taking into account that $P \geq \underline{P}$ and that it satisfies STP. Since this holds for any $\varepsilon>0$, we deduce that $\underline{P}\left(g-f-\inf _{\omega \in \Omega} \underline{P}\left(g-f^{\omega}\right)\right) \geq 0$ and as a consequence $\underline{P}$ also satisfies STP.

The next proof is a reformulation of one given by Galaabaatar and Karni (2013, Thm. 1).

Proof of Proposition 19. The direct implication follows from Lemma 18. Let us prove the converse.

Let $\exp (\mathcal{M}(\underline{P}))$ denote the set of exposed points of $\mathcal{M}(\underline{P}) \cdot{ }^{21}$ Since $\Omega, \mathcal{X}$ are finite, it follows from Straszewicz's theorem (see for instance Rockafellar, 1970, Thm. 18.6) that $\mathcal{M}(\underline{P})$ is the closed convex hull of $\exp (\mathcal{M}(\underline{P}))$, and as a consequence $\underline{P}$ is the lower envelope of the set of exposed points. Let us show that each of these linear previsions satisfies Eq. (23).

21. From the book by Rockafellar (1970, sec. 18), an exposed point of a convex set $\mathcal{C}$ is a point through which there is a supporting hyperplane which contains no other point of $\mathcal{C}$. 
In order to prove this, we are going to show first that the following condition is necessary for the factorisation in Eq. (23):

$$
\left(\forall x_{1}, x_{2} \in \mathcal{X}\right)\left(\exists k_{x_{1}, x_{2}} \in \mathbb{R}\right)(\forall \omega \in \Omega) P\left(\left\{\left(\omega, x_{1}\right)\right\}\right)=k_{x_{1}, x_{2}} P\left(\left\{\left(\omega, x_{2}\right)\right\}\right) .
$$

To prove that this is the case, note that under the above conditions we get, for any $\omega \in \Omega, x \in \mathcal{X}$, that

$$
P(\{\omega\})=\sum_{x^{\prime} \in \mathcal{X}} P\left(\left\{\left(\omega, x^{\prime}\right)\right\}\right)=\sum_{x^{\prime} \in \mathcal{X}} k_{x, x^{\prime}} P(\{(\omega, x)\})=P(\{(\omega, x)\})\left(\sum_{x^{\prime} \in \mathcal{X}} k_{x, x^{\prime}}\right)
$$

and

$$
\begin{aligned}
P(\{x\}) k_{x, x^{\prime}}=\left(\sum_{\omega^{\prime} \in \Omega} P\left(\left\{\left(\omega^{\prime}, x\right)\right\}\right)\right) k_{x, x^{\prime}} & =\sum_{\omega^{\prime} \in \Omega}\left(k_{x, x^{\prime}} P\left(\left\{\left(\omega^{\prime}, x\right)\right\}\right)\right) \\
& =\sum_{\omega^{\prime} \in \Omega} P\left(\left\{\left(\omega^{\prime}, x^{\prime}\right)\right\}\right)=P\left(\left\{x^{\prime}\right\}\right),
\end{aligned}
$$

whence

$$
P(\{x\})\left(\sum_{x^{\prime} \in \mathcal{X}} k_{x, x^{\prime}}\right)=\sum_{x^{\prime} \in \mathcal{X}} k_{x, x^{\prime}} P(\{x\})=\sum_{x^{\prime} \in \mathcal{X}} P\left(\left\{x^{\prime}\right\}\right)=1 .
$$

This implies that $P(\{x\})>0$ and as a consequence that $\left(\sum_{x^{\prime} \in \mathcal{X}} k_{x, x^{\prime}}\right)=\frac{1}{P(\{x\})}$; using Eq. (31), we conclude that $P(\{(\omega, x)\})=P(\{\omega\}) P(\{x\})$.

Now, assume ex-absurdo that there is an exposed point $P$ of $\mathcal{M}(\underline{P})$ that does not satisfy Eq. (23). Then the reasoning above implies that we can find two elements $x_{1}, x_{2}$ in $\mathcal{X}$ for which there is no real $k$ such that $P\left(\left\{\left(\omega, x_{1}\right)\right\}\right)=k P\left(\left\{\left(\omega, x_{2}\right)\right\}\right)$ for all $\omega$.

This in turn implies that there are $\omega_{1}, \omega_{2}$ in $\Omega$ such that $P\left(\left\{\left(\omega_{1}, x_{1}\right)\right\}\right)=k_{1} P\left(\left\{\left(\omega_{1}, x_{2}\right)\right\}\right)$ and $P\left(\left\{\left(\omega_{2}, x_{1}\right)\right\}\right)=k_{2} P\left(\left\{\left(\omega_{2}, x_{2}\right)\right\}\right)$ for different non-negative real numbers $k_{1} \neq k_{2}$. Note that the inequalities $P\left(\left\{\left(\omega_{1}, x_{1}\right)\right\}\right) \neq k_{2} P\left(\left\{\left(\omega_{1}, x_{2}\right)\right\}\right)$ and $P\left(\left\{\left(\omega_{2}, x_{1}\right)\right\}\right) \neq k_{1} P\left(\left\{\left(\omega_{2}, x_{2}\right)\right\}\right)$ imply that $P\left(\left\{\left(\omega_{1}, x_{2}\right)\right\}\right), P\left(\left\{\left(\omega_{2}, x_{2}\right)\right\}\right) \neq 0$ and that $k_{1}, k_{2}>0$. Assume without loss of generality that $k_{1}>k_{2}$. Then for every $\lambda \in\left(\frac{1}{k_{1}}, \frac{1}{k_{2}}\right)$,

$$
P\left(\lambda I_{\left\{x_{1}\right\}} \mid\left\{\omega_{1}\right\}\right)>P\left(I_{\left\{x_{2}\right\}} \mid\left\{\omega_{1}\right\}\right) \text { and } P\left(\lambda I_{\left\{x_{1}\right\}} \mid\left\{\omega_{2}\right\}\right)<P\left(I_{\left\{x_{2}\right\}} \mid\left\{\omega_{2}\right\}\right) .
$$

In particular, we can choose some $\lambda^{*} \in\left(\frac{1}{k_{1}}, \frac{1}{k_{2}}\right)$ such that $P\left(\lambda^{*} I_{\left\{x_{1}\right\}}\right) \neq P\left(I_{\left\{x_{2}\right\}}\right)$. Consider then the gambles $h_{1}:=\lambda^{*} I_{\left\{x_{1}\right\}}$ and $h_{2}:=I_{\left\{x_{2}\right\}}$.

Assume for instance that $P\left(h_{1}\right)>P\left(h_{2}\right)$ (if $P\left(h_{1}\right)<P\left(h_{2}\right)$ then it suffices to reverse the argument below), and let us define the gamble $f \in \mathcal{L}(\Omega \times \mathcal{X})$ by

$$
\begin{aligned}
f: \Omega \times \mathcal{X} & \rightarrow \mathbb{R} \\
(\omega, x) & \mapsto \begin{cases}h_{1}(\omega, x) & \text { if } P\left(h_{1} \mid\{\omega\}\right) \geq P\left(h_{2} \mid\{\omega\}\right), \\
h_{2}(\omega, x) & \text { otherwise. }\end{cases}
\end{aligned}
$$

It follows from this definition that

$$
f^{\omega}= \begin{cases}h_{1}^{\omega} & \text { if } P\left(h_{1} \mid\{\omega\}\right) \geq P\left(h_{2} \mid\{\omega\}\right), \\ h_{2}^{\omega} & \text { otherwise }\end{cases}
$$


whence $f^{\omega_{1}}=h_{1}^{\omega_{1}}, f^{\omega_{2}}=h_{2}^{\omega_{2}}$.

Now, since $P$ is an exposed point, there is a gamble $g \in \mathcal{L}(\mathcal{X} \times \Omega)$ such that $P(g)<P^{\prime}(g)$ for any $P^{\prime} \in \mathcal{M}(\underline{P}), P^{\prime} \neq P$. Note that we can assume without loss of generality that $P(g)=0$, simply by redefining $g:=g-P(g)$. In particular, for every $\lambda>0$ it holds that $\underline{P}(\lambda g)=\lambda \underline{P}(g)=0$, and therefore

$$
(\forall \lambda>0) \underline{P}\left(\lambda g+h_{1}-h_{1}\right)=\underline{P}\left(\lambda g+h_{1}-f^{\omega_{1}}\right)=0,
$$

taking into account that $h_{1}^{\omega_{1}}=h_{1}$ because it is an $\mathcal{X}$-measurable gamble.

Let us show that there is some $\lambda>0$ such that $\underline{P}\left(\lambda g+h_{1}-h_{2}\right) \geq 0$. If this were not the case, then for every natural number $n$ there would be some $P_{n} \in \mathcal{M}(\underline{P})$ such that $P_{n}\left(n g+h_{1}-h_{2}\right)<0$, whence $P_{n}\left(h_{2}-h_{1}\right)>n P_{n}(g)$. Since $\mathcal{M}(\underline{P})$ is a compact subset of $\mathbb{R}^{|\Omega \times \mathcal{X}|}$, which is a metric space, it is sequentially compact, so there is a subsequence of $\left(P_{n}\right)_{n \in \mathbb{N}}$ that converges towards some $P^{\prime} \in \mathcal{M}(\underline{P})$. If $P^{\prime}(g)>0$, then we have that $P^{\prime}\left(h_{2}-h_{1}\right) \geq m P^{\prime}(g)$ for all $m \in \mathbb{N}$, which means that $P^{\prime}\left(h_{2}-h_{1}\right)=+\infty$, a contradiction. Thus, we must have $P^{\prime}(g)=0$ or, equivalently, $P^{\prime}=P$. But $P\left(h_{2}-h_{1}\right)=P^{\prime}\left(h_{2}-h_{1}\right) \geq 0$ by construction, while we have assumed before that $P\left(h_{1}\right)>P\left(h_{2}\right)$. This is a contradiction.

We conclude that there is some $\lambda^{\star}>0$ such that $\underline{P}\left(\lambda^{\star} g+h_{1}-h_{2}\right) \geq 0$. Then $\underline{P}\left(\lambda^{\star} g+\right.$ $\left.h_{1}-f^{\omega}\right) \geq 0$ for all $\omega \in \Omega$, since we have established that

$$
\underline{P}\left(\lambda^{\star} g+h_{1}-h_{1}\right) \geq 0 \text { and } \underline{P}\left(\lambda^{\star} g+h_{1}-h_{2}\right) \geq 0 .
$$

Moreover,

$$
\begin{aligned}
& \underline{P}\left(\lambda^{\star} g+h_{1}-f\right) \leq P\left(\lambda^{\star} g+h_{1}-f\right)=P\left(h_{1}-f\right)=\sum_{\omega \in \Omega} P(\{\omega\})\left[P\left(h_{1} \mid\{\omega\}\right)-P\left(f^{\omega} \mid\{\omega\}\right)\right] \\
& =\sum_{\omega \in \Omega: f^{\omega}=h_{2}} P(\{\omega\})\left[P\left(h_{1} \mid\{\omega\}\right)-P\left(h_{2} \mid\{\omega\}\right)\right] \leq P\left(\left\{\omega_{2}\right\}\right)\left[P\left(h_{1} \mid\left\{\omega_{2}\right\}\right)-P\left(h_{2} \mid\left\{\omega_{2}\right\}\right)\right]<0,
\end{aligned}
$$

since $P\left(\left\{\omega_{2}\right\}\right)>0$ and $P\left(h_{1} \mid\left\{\omega_{2}\right\}\right)<P\left(h_{2} \mid\left\{\omega_{2}\right\}\right)$. This is a contradiction with STP.

The next one is a technical result needed in the proof of Proposition 20 below.

Lemma 33. Let $\underline{P}_{1}, \underline{P}_{2}$ be two coherent lower previsions with a linear domain $\mathcal{K} \subseteq \mathcal{L}$, and such that $\underline{P}_{1}(f) \geq \underline{P}_{2}(f)$ for every $f \in \mathcal{K}$. Let $\underline{P}_{2}^{\prime}$ be a coherent lower prevision on $\mathcal{L}$ that extends $\underline{P}_{2}$. Then there is a coherent lower prevision $\underline{P}_{1}^{\prime}$ on $\mathcal{L}$ that extends $\underline{P}_{1}$ such that $\underline{P}_{1}^{\prime} \geq \underline{P}_{2}^{\prime}$.

Proof. Consider the credal set $\mathcal{M}\left(\underline{P}_{1}\right):=\left\{P\right.$ linear : $\left.(\forall f \in \mathcal{K}) P(f) \geq \underline{P}_{1}(f)\right\}$, and let $\mathcal{M}\left(\underline{P}_{2}^{\prime}\right)$ be the credal set associated with $\underline{P}_{2}^{\prime}$. Define $\mathcal{M}:=\mathcal{M}\left(\underline{P}_{1}\right) \cap \mathcal{M}\left(\underline{P}_{2}^{\prime}\right)$. This is the intersection of two compact and convex sets, so it is also compact and convex. We begin by proving that it is non-empty.

Let us assume by contradiction that $\mathcal{M}$ is empty. Then we can apply the strong separation theorem by Walley (1991, app. E3) and find a gamble $f$ and a real number $\mu$ such that $P^{\prime}(f)<\mu$ for every $P^{\prime} \in \mathcal{M}\left(\underline{P}_{1}\right)$ and $P^{\prime}(f)>\mu$ for every $P^{\prime} \in \mathcal{M}\left(\underline{P}_{2}^{\prime}\right)$. This means that the upper envelope $\bar{E}_{\underline{P}_{1}}$ of $\mathcal{M}\left(\underline{P}_{1}\right)$ satisfies $\bar{E}_{\underline{P}_{1}}(f)<\underline{P}_{2}^{\prime}(f)$.

Since $\mathcal{K}$ is a linear set, we can regard it as a convex cone with the additional property that $\mathcal{K}=-\mathcal{K}$. This allows us to deduce from a theorem by Walley (1991, Thm. 3.1.4) that 
$\bar{E}_{\underline{P}_{1}}(f)=\inf \left\{\bar{P}_{1}(g)+\mu: f \leq g+\mu, g \in \mathcal{K}\right\}<\underline{P}_{2}^{\prime}(f)$; whence there is some $\varepsilon>0$, some real $\mu$ and some $g \in \mathcal{K}$ such that $f \leq g+\mu$ and $\bar{P}_{1}(g)+\mu \leq \underline{P}_{2}^{\prime}(f)-\varepsilon$. It follows that

$$
\begin{aligned}
f-g-\mu+\bar{P}_{1}(g)+\mu \leq \underline{P}_{2}^{\prime}(f)-\varepsilon & \Rightarrow f-\underline{P}_{2}^{\prime}(f)-g+\bar{P}_{1}(g) \leq-\varepsilon \\
& \Rightarrow f-\underline{P}_{2}^{\prime}(f)-g+\underline{P}_{2}^{\prime}(g) \leq-\varepsilon,
\end{aligned}
$$

taking into account that $\bar{P}_{1}(g) \geq \underline{P}_{1}(g) \geq \underline{P}_{2}(g)=\underline{P}_{2}^{\prime}(g)$, given that $g \in \mathcal{K}$ and that $\underline{P}_{2}^{\prime}$ is an extension of $\underline{P}_{2}$. This contradicts the coherence of $\underline{P}_{2}^{\prime}$. We conclude that $\mathcal{M}$ is non-empty.

Applying now a result by Walley (1991, Thm. 3.6.1), $\mathcal{M}$ induces a coherent lower prevision $\underline{P}_{1}^{\prime}$ on $\mathcal{L}$, and $\mathcal{M}\left(\underline{P}_{1}^{\prime}\right)=\mathcal{M}=\mathcal{M}\left(\underline{P}_{1}\right) \cap \mathcal{M}\left(\underline{P}_{2}^{\prime}\right)$. This implies that $\underline{P}_{1}^{\prime}$ dominates $\underline{P}_{2}^{\prime}$ as well as $\underline{P}_{1}$. In order to show that it is an extension of $\underline{P}_{1}$, it remains to prove that $\underline{P}_{1}^{\prime}$ coincides with $\underline{P}_{1}$ on $\mathcal{K}$. Consider a gamble $f \in \mathcal{K}$, and let $P_{1} \in \mathcal{M}\left(\underline{P}_{1}\right)$ satisfy $P_{1}(f)=\underline{P}_{1}(f)$. The restriction of $P_{1}$ to $\mathcal{K}$ dominates $\underline{P}_{2}$, so we can reason as before to conclude that $\mathcal{M}\left(P_{1}\right) \cap \mathcal{M}\left(\underline{P}_{2}^{\prime}\right) \neq \emptyset$.

Note that any linear prevision $P_{1}^{\prime}$ in $\mathcal{M}\left(P_{1}\right)$ must agree with $P_{1}$ on $\mathcal{K}$, since this set is negation invariant and $P_{1}^{\prime}(f) \geq P_{1}(f)$ and also $P_{1}(f)=-P_{1}(-f) \geq-P_{1}^{\prime}(-f)=P_{1}^{\prime}(f)$ for any $f \in \mathcal{K}$. Thus, we conclude that there is a linear prevision $P_{1}^{\prime}$ that coincides with $P_{1}$ in $\mathcal{K}$ and dominates $\underline{P}_{2}^{\prime}$ on all gambles. It follows that $P_{1}^{\prime} \in \mathcal{M}\left(\underline{P}_{1}\right) \cap \mathcal{M}\left(\underline{P}_{2}^{\prime}\right)=\mathcal{M}\left(\underline{P}_{1}^{\prime}\right)$. As a consequence, $\underline{P}_{1}^{\prime}(f) \leq P_{1}^{\prime}(f)=\underline{P}_{1}(f)$, and since the converse inequality holds trivially we conclude that $\underline{P}_{1}^{\prime}=\underline{P}_{1}$ on $\mathcal{K}$.

Proof of Proposition 20. If $\Omega$ is finite, the result follows from Proposition 19. Consider next the case of $\Omega$ infinite, and assume ex-absurdo that there is a gamble $f$ on $\Omega \times \mathcal{X}$ such that $\underline{P}(f)<\left(\underline{P}_{\Omega}^{\prime} \otimes \underline{P}_{\mathcal{X}}^{\prime}\right)(f)$. By Lemma 18 , STP is preserved by taking lower envelopes, so we can redefine $\underline{P}:=\min \left\{\underline{P}, \underline{P}_{\Omega}^{\prime} \otimes \underline{P}_{\mathcal{X}}^{\prime}\right\}$ and obtain a coherent lower prevision on $\mathcal{L}(\Omega \times \mathcal{X})$ that is dominated by $\underline{P}_{\Omega}^{\prime} \otimes \underline{P}_{\mathcal{X}}^{\prime}$ and satisfies STP.

Let us prove that for every natural number $n$ there exists a simple gamble $f_{n}$ on $\Omega \times \mathcal{X}$ such that $\left\|f_{n}-f\right\| \leq \frac{1}{n}$.

Since $f$ is bounded, there exists some natural number $k$ such that $-k \leq \inf f \leq \sup f \leq$ $k$. For every $n$, there exists a finite partition $\mathcal{B}_{n}$ of $[-k, k]$ with intervals $I_{j}^{n}$ of length smaller than $\frac{1}{n}$. Let us define the gamble $f_{n}$ on $\Omega \times \mathcal{X}$ by

$$
f_{n}(\omega, x):=\inf I_{j}^{n} \quad \text { if } f(\omega, x) \in I_{j}^{n}
$$

for every $(\omega, x) \in \Omega \times \mathcal{X}$. Then since the partition $\mathcal{B}_{n}$ is finite, we deduce that $f_{n}$ is simple. Moreover, by construction $\left|f_{n}(\omega, x)-f(\omega, x)\right| \leq \frac{1}{n}$ for every $(\omega, x) \in \Omega \times \mathcal{X}$.

Let us define next the relation

$$
\omega \sim \omega^{\prime} \Leftrightarrow(\forall x \in \mathcal{X}) f_{n}(\omega, x)=f_{n}\left(\omega^{\prime}, x\right) .
$$

This is trivially an equivalence relation. It has a finite number of equivalence classes: if $\left\{z_{1}, \ldots, z_{k}\right\}$ denotes the finite range of $f_{n}$ and $\mathcal{X}=\left\{x_{1}, \ldots, x_{m}\right\}$, then the equivalence classes are given by

$$
\left\{\omega:(\forall i=1, \ldots, m) f_{n}\left(\omega, x_{i}\right)=z_{t_{i}}\right\}
$$

where $\left(t_{1}, \ldots, t_{m}\right) \in\{1, \ldots, k\}^{m}$ is finite. In other words, the number of equivalence classes is at most $\{1, \ldots, k\}^{m}$. Let us denote these classes by $\left\{B_{1}, \ldots, B_{\ell}\right\}$. 
Next, let us define the set of gambles

$$
\mathcal{K}:=\left\{g \in \mathcal{L}(\Omega \times \mathcal{X}):\left(\forall \omega \sim \omega^{\prime}\right) g(\omega, x)=g\left(\omega^{\prime}, x\right)\right\} .
$$

This is a linear set of gambles: given $g_{1}, g_{2} \in \mathcal{K}$ and reals $\lambda_{1}, \lambda_{2}$, it holds for every $\omega \sim \omega^{\prime}$ that

$$
\begin{aligned}
\left(\lambda_{1} g_{1}+\lambda_{2} g_{2}\right)(\omega, x) & =\lambda_{1} g_{1}(\omega, x)+\lambda_{2} g_{2}(\omega, x) \\
& =\lambda_{1} g_{1}\left(\omega^{\prime}, x\right)+\lambda_{2} g_{2}\left(\omega^{\prime}, x\right)=\left(\lambda_{1} g_{1}+\lambda_{2} g_{2}\right)\left(\omega^{\prime}, x\right) .
\end{aligned}
$$

The set $\mathcal{K}$ corresponds to the gambles that are measurable with respect to the finite partition $\left\{B_{j} \times\left\{x_{i}\right\}: i=1, \ldots, m, j=1, \ldots, \ell\right\}$ of $\Omega \times \mathcal{X}$, and by construction it includes the gamble $f_{n}$.

Let $\underline{Q}$ denote the restriction of $\underline{P}$ to $\mathcal{K}$. Its marginals are $\underline{Q}_{\Omega}^{\prime}$ and $\underline{Q}_{\mathcal{X}}^{\prime}$, where $\underline{Q}_{\Omega}^{\prime}$ is the restriction of $\underline{P}_{\Omega}^{\prime}$ to the gambles that are measurable with respect to the partition $\mathcal{B}:=$ $\left\{B_{1}, \ldots, B_{\ell}\right\}$ of $\Omega$. Moreover, $\mathcal{K}$ is in a one-to-one correspondence with $\mathcal{L}(\{1, \ldots, \ell\} \times \mathcal{X})$, so we can regard $Q$ as a coherent lower prevision defined on the set of gambles on a finite possibility space. Since $\underline{P}$ satisfies STP, so does its restriction $\underline{Q}$. By Proposition $19, \underline{Q}$ is a lower envelope of linear previsions satisfying Eq. (23), so there are two linear previsions $Q_{\Omega}^{\prime} \geq \underline{Q}_{\Omega}^{\prime}, Q_{\mathcal{X}}^{\prime} \geq \underline{Q}_{\mathcal{X}}^{\prime}$ such that $\underline{P}\left(f_{n}\right)=\underline{Q}\left(f_{n}\right)=\left(Q_{\Omega}^{\prime} \times Q_{\mathcal{X}}^{\prime}\right)\left(f_{n}\right)$. By construction, we can regard $Q_{\Omega}^{\prime}$ as a linear prevision defined on the set $\left\{f \in \mathcal{L}(\Omega):\left(\forall \omega \sim \omega^{\prime}\right) f(\omega)=f\left(\omega^{\prime}\right)\right\}$ that dominates $\underline{P}_{\Omega}^{\prime}$ on this domain. By Lemma 33, there is a linear prevision $P_{\Omega}^{\prime} \in \mathcal{M}\left(\underline{P}_{\Omega}^{\prime}\right)$ that extends $Q_{\Omega}^{\prime}$. This means that $\underline{P}\left(f_{n}\right)=\left(Q_{\Omega}^{\prime} \times Q_{\mathcal{X}}^{\prime}\right)\left(f_{n}\right) \geq\left(\underline{P}_{\Omega}^{\prime} \otimes \underline{P}_{\mathcal{X}}^{\prime}\right)\left(f_{n}\right)$, and applying coherence, that $\underline{P}(f)=\lim _{n \rightarrow \infty} \underline{P}\left(f_{n}\right) \geq \lim _{n \rightarrow \infty}\left(\underline{P}_{\Omega}^{\prime} \otimes \underline{P}_{\mathcal{X}}^{\prime}\right)\left(f_{n}\right)=\left(\underline{P}_{\Omega}^{\prime} \otimes \underline{P}_{\mathcal{X}}^{\prime}\right)(f)$. Since $\underline{P}$ is dominated by the strong product $\underline{P}_{\Omega}^{\prime} \otimes \underline{P}_{\mathcal{X}}^{\prime}$, we conclude that $\underline{P}(f)=\left(\underline{P}_{\Omega}^{\prime} \otimes \underline{P}_{\mathcal{X}}^{\prime}\right)(f)$, a contradiction.

Proof of Corollary 21. The equivalence between (i) and (ii) follows from Proposition 17, and the equivalence between (i) and (iii) is a consequence of Lemma 18.

Proof of Theorem 22. The proof follows from Proposition 17 and Lemma 18 for the direct implication, and from Propositions 17 and 20 for the converse one.

Proof of Proposition 23. (i) $(\Rightarrow)$ Consider $f, g \in \mathcal{C}$. Then we can express them as $f=\lambda_{1}\left(p_{1}-q_{1}\right), g=\lambda_{2}\left(p_{2}-q_{2}\right)$, for $\lambda_{1}, \lambda_{2}>0, p_{1} \succ q_{1}, p_{2} \succ q_{2}$. Assume for the time being that $\lambda_{1}=\lambda_{2}=1$. By A2,

$$
\alpha p_{1}+(1-\alpha) p_{2} \succ \alpha q_{1}+(1-\alpha) p_{2} \succ \alpha q_{1}+(1-\alpha) q_{2}
$$

for any $\alpha \in(0,1)$. Applying now A3, we deduce the existence of $\beta_{1}, \beta_{2} \in(0,1)$ such that

$$
\begin{aligned}
& \beta_{1}\left(\alpha p_{1}+(1-\alpha) p_{2}\right)+\left(1-\beta_{1}\right)\left(\alpha q_{1}+(1-\alpha) q_{2}\right) \succ \alpha q_{1}+(1-\alpha) p_{2} \text { and } \\
& \alpha q_{1}+(1-\alpha) p_{2} \succ \beta_{2}\left(\alpha p_{1}+(1-\alpha) p_{2}\right)+\left(1-\beta_{2}\right)\left(\alpha q_{1}+(1-\alpha) q_{2}\right),
\end{aligned}
$$

from which we deduce that

$$
\begin{aligned}
& \alpha \beta_{1}\left(p_{1}-q_{1}\right)-(1-\alpha)\left(1-\beta_{1}\right)\left(p_{2}-q_{2}\right) \in \mathcal{C} \text { and } \\
& (1-\alpha)\left(1-\beta_{2}\right)\left(p_{2}-q_{2}\right)-\alpha \beta_{2}\left(p_{1}-q_{1}\right) \in \mathcal{C}
\end{aligned}
$$


normalising we find $\gamma_{1}, \gamma_{2} \in(0,1)$ such that

$$
\gamma_{1} f-\left(1-\gamma_{1}\right) g \in \mathcal{C} \text { and } \gamma_{2} g-\left(1-\gamma_{2}\right) f \in \mathcal{C} .
$$

Next, if either $\lambda_{1}$ or $\lambda_{2} \neq 1$, we deduce from the reasoning above that there is some $\beta \in(0,1)$ such that

$$
\beta \frac{f}{\lambda_{1}}-(1-\beta) \frac{g}{\lambda_{2}} \in \mathcal{C}
$$

whence, given $k:=\beta+(1-\beta) \frac{\lambda_{1}}{\lambda_{2}}$, it holds that

$$
\beta \frac{f}{\lambda_{1}} \frac{\lambda_{1}}{k}-(1-\beta) \frac{g}{\lambda_{2}} \frac{\lambda_{1}}{k} \in \mathcal{C},
$$

and $\beta_{1}:=\frac{\beta}{k} \in(0,1)$. Analogously, we can find $\beta_{2} \in(0,1)$ such that $\beta_{2} g-(1-$ $\left.\beta_{2}\right) f \in(0,1)$.

$(\Leftarrow)$ Given $p \succ q \succ r$, it follows from Eq. (12) that $f:=p-q, g:=q-r \in \mathcal{C}$. Applying A3, there are $\beta_{1}, \beta_{2} \in(0,1)$ such that $\beta_{1} f-\left(1-\beta_{1}\right) g \in \mathcal{C}, \beta_{2} g-(1-$ $\left.\beta_{2}\right) f \in \mathcal{C}$. But taking into account that

$\beta_{1} f-\left(1-\beta_{1}\right) g=\beta_{1} p+\left(1-\beta_{1}\right) r-q$ and $\beta_{2} g-\left(1-\beta_{2}\right) f=q-\beta_{2} r-\left(1-\beta_{2}\right) p$, we deduce from Proposition 1 that $\beta_{1} p+\left(1-\beta_{1}\right) r \succ q \succ \beta_{2} r+\left(1-\beta_{2}\right) p$.

(ii) $(\Rightarrow)$ Consider $f \in \mathcal{C}, g \in \mathcal{A}$. Then there are $\lambda_{1}, \lambda_{2}>0, p \succ q$ and $r, s \in \mathcal{H}$ such that $f=\lambda_{1}(p-q), g=\lambda_{2}(r-s)$. Assume first of all that $\lambda_{1}=\lambda_{2}=1$. Then there is some $\beta \in(0,1)$ such that $\beta p+(1-\beta) r \succ \beta q+(1-\beta) s$, whence $\beta f+(1-\beta) g \in \mathcal{C}$. Now, if either $\lambda_{1}, \lambda_{2} \neq 1$, it follows from the above reasoning that there is some $\beta \in(0,1)$ such that $\beta \frac{f}{\lambda_{1}}+(1-\beta) \frac{g}{\lambda_{2}} \in \mathcal{C}$, whence $\beta f+\frac{(1-\beta) \lambda_{1}}{\lambda_{2}} g \in \mathcal{C}$, and, by considering the normalising constant $k:=\beta+\frac{(1-\beta) \lambda_{1}}{\lambda_{2}}$, it follows that, given $\beta^{\prime}:=\frac{\beta}{k} \in(0,1), \beta^{\prime} f+\left(1-\beta^{\prime}\right) g \in \mathcal{C}$.

$(\Leftarrow)$ Consider $p \succ q$ and take $r, s \in \mathcal{H}$. Then $f:=p-q \in \mathcal{C}, g:=r-s \in \mathcal{A}$, whence there is some $\beta \in(0,1)$ such that $\beta f+(1-\beta) g=\beta p+(1-\beta) r-\beta q-(1-\beta) s \in \mathcal{C}$. Applying Proposition 1, we deduce that $\beta p+(1-\beta) r \succ \beta q+(1-\beta) s$.

(iii) Consider $f, g \in \mathcal{C}$. Since $-g,-f \in \mathcal{A}$, we deduce from the second statement that there are $\beta_{1}, \beta_{2} \in(0,1)$ such that $\beta_{1} f-\left(1-\beta_{1}\right) g \in \mathcal{C}$ and $\beta_{2} g-\left(1-\beta_{2}\right) f \in \mathcal{C}$. Applying the first statement, we deduce that $\succ$ satisfies A3. This completes the proof.

Proof of Proposition 24. (i $\Rightarrow$ ii) This case has been proven in Proposition 23.

(i $\Leftarrow \mathrm{ii})$ Consider $p, q, r, s \in \mathcal{H}_{z}$ such that $p \succ q$. Observe first of all that the following is true for some $\alpha \in(0,1)$ :

$$
\alpha p+(1-\alpha) z \succ \alpha q+(1-\alpha) s .
$$

This is trivial if $s=z$ and otherwise it follows from Proposition 23 through $\alpha(p-q)-$ $(1-\alpha)(s-z) \in \mathcal{C}$. It shows in particular that the case $r=z$ holds. Therefore assume that $r \neq z$. Apply A2 to obtain that $\alpha p+(1-\alpha) r \succ \alpha p+(1-\alpha) z$. Combining this with (32), we conclude that $\alpha p+(1-\alpha) r \succ \alpha q+(1-\alpha) s$. 
(ii $\Rightarrow$ iii) By using the equivalence between wA3 and A3' (see Proposition 7), we deduce that A3 implies wA3. Thus it is enough to have that $\underline{P}(f)>0$ for all $f \in \mathcal{L}^{+}(\Omega \times \mathcal{X})$; this is equivalent to showing that for every $(\omega, x) \in \Omega \times \mathcal{X}$ there is some $\delta>0$ such that $I_{\{(\omega, x)\}}-\delta \in \mathcal{R}$.

Let $u$ be the uniform act on $\Omega \times \mathcal{X}_{z}$, and let $p$ be the horse lottery given by $p:=$ $u+\frac{1}{\left|\mathcal{X}_{z}\right|}\left(I_{\{(\omega, x)\}}-I_{\{(\omega, z)\}}\right)$. Then it follows that $p \succ u \succ z$. Applying A3, we deduce that there is some $\alpha \in(0,1)$ such that $\alpha p+(1-\alpha) z \succ u$, whence

$$
\psi(\alpha p+(1-\alpha) z-u)=(2 \alpha-1) \frac{1}{\left|\mathcal{X}_{z}\right|} I_{\{(\omega, x)\}}-(1-\alpha) \frac{1}{\left|\mathcal{X}_{z}\right|} I_{\{(\omega, x)\}^{c}} \in \mathcal{R}
$$

From this it follows that $2 \alpha-1>0$, and applying the non-negative homogeneity of $\mathcal{R}$ we deduce that there is some $\delta>0$ such that $I_{\{(\omega, x)\}}-\delta I_{\{(\omega, x)\}^{c}} \in \mathcal{R}$. Consider now a natural number $N>\delta$. Then

$$
\begin{aligned}
I_{\{(\omega, x)\}}- & \frac{\delta}{N+1}= \\
& \quad\left(\frac{1}{N+1} I_{\{(\omega, x)\}}-\frac{\delta}{N+1} I_{\{(\omega, x)\}^{c}}\right)+\left(\frac{N}{N+1}-\frac{\delta}{N+1}\right) I_{\{(\omega, x)\}} \in \mathcal{R} .
\end{aligned}
$$

(ii $\Leftarrow$ iii) Thanks to Proposition 7 , we know that wA3 implies A3 as long as the latter is applied to non-trivial preferences. Let us show that the positivity condition implies the case with objective preferences involved. In particular, let us consider $p \triangleright q \succ r$ (the remaining cases are analogous). By the super-additivity and positive homogeneity of coherent lower previsions, and for any $\alpha \in(0,1)$, we have that $\underline{P}(\alpha \psi(p-q)+$ $(1-\alpha) \psi(r-q)) \geq \alpha \underline{P}(\psi(p-q))+(1-\alpha) \underline{P}(\psi(r-q))$; and this is positive for small enough $1-\alpha$, considered that $\underline{P}(\psi(p-q))>0$ by assumption. This implies that $\alpha \psi(p-q)+(1-\alpha) \psi(r-q) \in \mathcal{R}$, or, in other words, that $\alpha p+(1-\alpha) r \succ$ $\alpha q+(1-\alpha) q=q$.

Lemma 34. Assume $\Omega$ is finite, and let $\succ$ be a coherent relation on $\mathcal{H} \times \mathcal{H}$. Let $\mathcal{R}$, from (15), be its associated coherent set of desirable gambles, and $\underline{P}$ its corresponding coherent lower prevision.

(i) $\mathcal{R}$ has an open superset $\Leftrightarrow(\forall f \in \mathcal{C}) \bar{P}(f)>0$.

(ii) If $\succ$ satisfies sA3, then: $(\exists f \in \mathcal{C}) \bar{P}(f)=0 \Rightarrow(\forall g \in \mathcal{A}) \underline{P}(g)=\bar{P}(g)=0$.

Proof. (i) We begin with the direct implication. Let $\mathcal{R}^{\prime}$ be an open superset of $\mathcal{R}$, and let $\underline{P}^{\prime}$ be its associated coherent lower prevision. The inclusion $\mathcal{R} \subseteq \mathcal{R}^{\prime}$ implies that $\underline{P}(f) \leq \underline{P}^{\prime}(f) \leq \bar{P}^{\prime}(f) \leq \bar{P}(f)$ for any gamble $f$. Moreover, for any $f \in \mathcal{R} \backslash \mathcal{L}^{+} \subseteq$ $\mathcal{R}^{\prime} \backslash \mathcal{L}^{+}$, it holds that $\underline{P}^{\prime}(f)>0$ because $\mathcal{R}^{\prime}$ is a coherent set of strictly desirable gambles, and as a consequence also $\bar{P}(f)>0$ for every $f \in \mathcal{C}$.

Conversely, taking into account that linear previsions on $\mathcal{L}(\Omega \times \mathcal{X})$ are characterized by their restrictions to events, the credal set $\mathcal{M}(\underline{P})$ can be regarded as a closed and convex subset of the Euclidean space. 
Take $P$ in the relative interior of $\mathcal{M}(\underline{P})$. Then there is some $\varepsilon>0$ such that $B(P ; \varepsilon) \cap$ $\operatorname{aff}(\mathcal{M}(\underline{P})) \subseteq \mathcal{M}(\underline{P})$, where aff $(\mathcal{M}(\underline{P}))$ denotes the affine expansion of $\mathcal{M}(\underline{P})$. We are going to prove that $P(f)>0$ for any $f \in \mathcal{C}$. Fix $f \in \mathcal{C}$. Using point (iii) in Theorem 9 , $\underline{P}(f)=0<\bar{P}(f)$. Assume ex-absurdo that $P(f)=0$.

Then for any other $P_{1} \neq P$ in $B(P ; \varepsilon)$ we can consider $P_{2}:=2 P-P_{1} \in \operatorname{aff}(\mathcal{M}(\underline{P})) \cap$ $B(P ; \varepsilon)$ : to prove that indeed $P_{2} \in B(P ; \varepsilon)$, note that $\left|P_{2}(\{\omega, x\})-P(\{\omega, x\})\right|=$ $\left|P(\{\omega, x\})-P_{1}(\{\omega, x\})\right| \leq \varepsilon$. Now, $P(f)=\underline{P}(f)=0$ implies that $P_{1}(f)=P_{2}(f)=0$, and with this reasoning we would conclude that $P^{\prime}(f)=0$ for every $P^{\prime} \in B(P ; \varepsilon)$.

However, since $f \neq 0$ because $\succ$ is irreflexive, there must be some $\omega \in \Omega$ such that $f(\omega, \cdot)$ is not constant on 0 , and then we deduce that there must be some $x_{i} \neq x_{j} \in \mathcal{X}$ such that

$$
f\left(\omega, x_{i}\right) \neq f\left(\omega, x_{j}\right) \text { and } \max \left\{P\left(\left\{\left(\omega, x_{i}\right)\right\}\right), P\left(\left\{\left(\omega, x_{j}\right)\right\}\right)\right\}>0
$$

to prove this, note that there must be some $x^{*} \in \mathcal{X}$ such that $P\left(\left\{\left(\omega, x^{*}\right)\right\}\right)>0$, and then if Eq. (33) did not hold we would obtain that $f\left(\omega, x^{*}\right)=f\left(\omega, x^{\prime}\right)$ for every $x^{\prime} \neq x^{*}$; since $\sum_{x \in \mathcal{X}} f(\omega, x)=0$, this would mean that $f(\omega, \cdot)$ is constant on 0 , a contradiction.

Consider then $x_{i}, x_{j}$ in the conditions of Eq. (33), and assume without loss of generality that $P\left(\left\{\left(\omega, x_{j}\right)\right\}\right)>0$. Then for $\varepsilon^{\prime}$ small enough $P^{\prime}:=P+\varepsilon^{\prime}\left(I_{\left\{\left(\omega, x_{i}\right)\right\}}-I_{\left\{\left(\omega, x_{j}\right)\right\}}\right)$ belongs to $B(P ; \varepsilon)$ and $P^{\prime}(f) \neq P(f)$, a contradiction.

Thus, we conclude that there is some linear prevision $P \in \mathcal{M}(\underline{P})$ satisfying $P(f)>0$ for every $f \in \mathcal{C}$. It follows that its associated set of strictly desirable gambles includes $\mathcal{R}$.

(ii) Assume $\underline{P}(f)=\bar{P}(f)=0$ for some $f \in \mathcal{C}$, and take $g \neq f$ in $\mathcal{A}$. Since $\succ$ satisfies sA3, we deduce from Proposition 23 that there is some $\beta \in(0,1)$ such that $\beta f+(1-\beta) g \in \mathcal{C}$, whence $0 \leq \underline{P}(\beta f-(1-\beta) g) \leq \bar{P}(\beta f)+\underline{P}((1-\beta) g)=0-(1-\beta) \underline{P}(g) \leq 0$, whence also $\underline{P}(g)=0$. Applying the same reasoning to $-g$ we conclude that $\underline{P}$ is constant on 0 in $\mathcal{A}$.

Proof of Proposition 25. Let $\underline{P}$ be the coherent lower prevision induced by $\mathcal{R}$, and assume ex-absurdo that $\mathcal{R}$ has no open superset. From Lemma 34, it follows that $\underline{P}(f)=$ $\bar{P}(f)=0$ for every $f \in \mathcal{A}$. Let us show that this leads to a contradiction.

For every $f \in \mathcal{A}, \underline{P}(f)=0$ implies that for every $\varepsilon>0$ there is some $g_{\varepsilon} \in \mathcal{C}$ such that $f+\varepsilon \geq g_{\varepsilon}$, or, equivalently, that $g_{\varepsilon}-f \leq \varepsilon$. If there were a pair $(\omega, x)$ such that $g_{\varepsilon}(\omega, x)-f(\omega, x)<-\varepsilon|\Omega \times \mathcal{X}|$, then it would be impossible to satisfy the constraint that $\sum_{(\omega, x) \in \Omega \times \mathcal{X}} f(\omega, x)=\sum_{(\omega, x) \in \Omega \times \mathcal{X}} g_{\varepsilon}(\omega, x)=0$, taking into account that $g_{\varepsilon}-f \leq \varepsilon$. Therefore, we obtain that $\left\|f-g_{\varepsilon}\right\| \leq \varepsilon|\Omega \times \mathcal{X}|$, where $\|\cdot\|$ denotes the supremum norm. As a consequence, $\mathcal{A}=\overline{\mathcal{C}}$, where the closure is taken in the topology of uniform convergence.

Let now $\psi^{\prime}$ denote the projection operator from $\Omega \times \mathcal{X}$ to $\Omega \times \mathcal{X}^{\prime}$, where $\mathcal{X}^{\prime}$ results from dropping the last component in $\mathcal{X}$. It follows that $\psi^{\prime}(\mathcal{A})=\mathbb{R}^{\left|\Omega \times \mathcal{X}^{\prime}\right|}$. In addition, since $\mathcal{C}$ is convex we deduce that $\psi(\mathcal{C})$ is also convex, and also $\overline{(\psi(\mathcal{C}))}=\psi(\overline{\mathcal{C}})=\mathbb{R}^{\left|\Omega \times \mathcal{X}^{\prime}\right|}$. But the only convex set that is dense in an Euclidean space is the Euclidean space itself, applying a result by Cheridito (2013, Cor. 1.2.13), whence $\psi(\mathcal{C})=\psi(\mathcal{A})$, and therefore $\mathcal{C}=\mathcal{A}$. But 
this would mean that for every $f \in \mathcal{A}$ both $f,-f \in \mathcal{C}$, which contradicts the coherence of the set $\mathcal{R}$.

Thus, $\bar{P}(f)>0$ for every $f \in \mathcal{C}$ and as a consequence $\mathcal{R}$ has an open superset.

Lemma 35. Assume that $\Omega$ is finite, and let $k:=|\mathcal{X}|-1$. Then the set $\mathcal{A}$ has a finite generating family $\left\{g_{1}, \ldots, g_{\ell}\right\}$ such that, if $\|f\| \leq \varepsilon$, then we can write $f=\sum_{i=1}^{\ell} \lambda_{i} g_{i}$ and $\lambda_{i} \in[0, k \varepsilon]$ for $i=1, \ldots, \ell$.

Proof. Denote the elements of $\mathcal{X}$ by $x_{1}, \ldots, x_{k+1}$ and consider the family $\left\{I_{\left\{\left(\omega, x_{i}\right)\right\}}-I_{\left\{\left(\omega, x_{j}\right)\right\}}\right.$ : $\left.\omega \in \Omega, x_{i} \neq x_{j} \in \mathcal{X}\right\}$, and let $f \in \mathcal{A}$. Let us show that we can express $f$ as a linear combination, with non-negative coefficients, of the functions in the generating family. To this end, we can work independently on each $\omega$.

Fix then $\omega \in \Omega$. In order to simplify the notation, in the following we rename $f_{\omega}:=$ $f(\omega, \cdot)$ and drop any other reference to $\omega$. Moreover, we assume without loss of generality that all the non-negative values of $f_{\omega}$ occur prior to the negative ones; that is, that $f_{\omega}\left(x_{i}\right) \geq 0$ for $i=1, \ldots, m$ and $f_{\omega}\left(x_{i}\right)<0$ for $i>m$. Let us show then that

$$
f_{\omega}=\sum_{i=1}^{k}\left[\left(I_{\left\{x_{i}\right\}}-I_{\left\{x_{i+1}\right\}}\right) \sum_{j=1}^{i} f_{\omega}\left(x_{i}\right)\right] .
$$

Observe first that the coefficients $\lambda_{i}:=\sum_{j=1}^{i} f_{\omega}\left(x_{i}\right)$ are indeed non-negative, thanks to the assumption that the non-negative values occur prior to the remaining ones, and because $f \in \mathcal{A}$. It trivially holds also that $\lambda_{i} \leq k \varepsilon$.

Now, to show that Eq. (34) holds, it is convenient to rewrite it as follows:

$$
f_{\omega}=\lambda_{1} I_{\left\{x_{1}\right\}}+\left[\sum_{i=2}^{k}\left(\lambda_{i}-\lambda_{i-1}\right) I_{\left\{x_{i}\right\}}\right]-\lambda_{k} I_{\left\{x_{k+1}\right\}} .
$$

Then it is trivial that (35) holds in the case of $x_{1}$. In case $i \in\{2, \ldots, k\}$, it is enough to observe that $\lambda_{i}-\lambda_{i-1}=\sum_{j=1}^{i} f_{\omega}\left(x_{i}\right)-\sum_{j=1}^{i-1} f_{\omega}\left(x_{i}\right)=f_{\omega}\left(x_{i}\right)$. As a consequence, the case of $x_{k+1}$ becomes trivial as well, given that $\sum_{i=1}^{k+1} f_{\omega}\left(x_{i}\right)=0$.

Lemma 36. Let $\succ$ be a coherent preference relation on $\mathcal{H} \times \mathcal{H}$ satisfying sA3 and with corresponding cone $\mathcal{C}$. Assume $\Omega$ is finite, and let $\mathcal{R}$ be given by Eq. (15). Then:

(i) For any gamble $f \in \mathcal{C}$, there exists some $\varepsilon_{f}>0$ such that the open ball $B\left(f ; \varepsilon_{f}\right)$ satisfies

$$
B\left(f ; \varepsilon_{f}\right) \cap \mathcal{A} \subseteq \mathcal{C}
$$

(ii) $\operatorname{posi}(\widetilde{\mathcal{R}}) \cap \mathcal{A}=\operatorname{posi}(\widetilde{\mathcal{R}} \cap \mathcal{A})$, where $\widetilde{\mathcal{R}}:=\cup_{f \in \mathcal{C}} B\left(f ; \varepsilon_{f}\right)$ and posi denotes the conic hull operation.

Proof. (i) Let $\underline{P}$ be the coherent lower prevision induced by $\mathcal{R}$. Let $f:=p-q$, and $\left\{g_{1}, \ldots, g_{\ell}\right\}$ be the finite generating family of $\mathcal{A}$, existing by Lemma 35. By Proposition 23 , for any $i=1, \ldots, \ell$, there is some $\beta_{i} \in(0,1)$ such that $\beta_{i} f+\left(1-\beta_{i}\right) g_{i} \in \mathcal{C}$. Moreover, since $f \in \mathcal{C}$ it follows that for any $\beta \geq \beta_{i}$ the gamble $\beta f+(1-\beta) g_{i}$ 
also belongs to $\mathcal{C}$. As a consequence, given $\beta^{*}:=\max _{i=1, \ldots, \ell} \beta_{i} \in(0,1)$, it holds that $\beta^{*} f+\left(1-\beta^{*}\right) g_{i} \in \mathcal{C}$ for all $i=1, \ldots, \ell$. Now, since $\mathcal{C}$ is a convex cone, given $\alpha_{i} \geq 0$ such that $\sum_{i=1}^{\ell} \alpha_{i}>0$,

$$
\sum_{i=1}^{\ell} \alpha_{i}\left(\beta^{*} f+\left(1-\beta^{*}\right) g_{i}\right)=\beta^{*}\left(\sum_{i=1}^{\ell} \alpha_{i}\right) f+\left(1-\beta^{*}\right) \sum_{i=1}^{\ell} \alpha_{i} g_{i} \in \mathcal{C} .
$$

Consider $\varepsilon<\frac{1-\beta^{*}}{\beta^{*} k \ell}$. By Lemma 35, for every $g \in \mathcal{A}$ such that $\|g-f\| \leq \varepsilon$, there are $\lambda_{i} \in[0, \varepsilon k]$ such that $g-f=\sum_{i=1}^{\ell} \lambda_{i} g_{i}$, whence

$$
g=f+\sum_{i=1}^{\ell} \lambda_{i} g_{i}=f+\frac{1-\beta^{*}}{\beta^{*}} \sum_{i=1}^{\ell}\left(\frac{\lambda_{i} \beta^{*}}{1-\beta^{*}}\right) g_{i}
$$

recall that $\|\cdot\|$ refers to the supremum norm. Applying Eq. (36) with $\alpha_{i}:=\frac{\lambda_{i} \beta^{*}}{1-\beta^{*}}$ and $\delta:=\sum_{i=1}^{\ell} \alpha_{i}>0$, we deduce that

$$
\beta^{*} \delta f+\left(1-\beta^{*}\right) \sum_{i=1}^{\ell} \frac{\lambda_{i} \beta^{*}}{1-\beta^{*}} g_{i}=\beta^{*} \delta f+\sum_{i=1}^{\ell} \lambda_{i} \beta^{*} g_{i} \in \mathcal{C},
$$

and since this set is a cone,

$$
\delta f+\sum_{i=1}^{\ell} \lambda_{i} g_{i} \in \mathcal{C} .
$$

Observe that by construction, $0<\delta=\sum_{i=1}^{\ell} \frac{\lambda_{i} \beta^{*}}{1-\beta^{*}} \leq \frac{\ell k \varepsilon \beta^{*}}{1-\beta^{*}}<1$, whence also $(1-\delta) f \in$ $\mathcal{C}$; and since this set is convex, we deduce that

$$
f+\sum_{i=1}^{\ell} \lambda_{i} g_{i}=g \in \mathcal{C} .
$$

We conclude that there is some $\varepsilon>0$ such that $g \in \mathcal{C}$ for every $g$ satisfying $\|g-f\| \leq \varepsilon$.

(ii) We immediately have that $\operatorname{posi}(\widetilde{\mathcal{R}} \cap \mathcal{A}) \subseteq \operatorname{posi}(\widetilde{\mathcal{R}}) \cap \operatorname{posi}(\mathcal{A})=\operatorname{posi}(\widetilde{\mathcal{R}}) \cap \mathcal{A}$, so we only need to establish the converse inclusion.

Let us begin by showing that $\widetilde{\mathcal{R}}$ is a cone. Consider a gamble $g \in \widetilde{\mathcal{R}}$ and $\lambda>0$. Then there is some $f \in \mathcal{C}$ such that $g \in B\left(f ; \varepsilon_{f}\right)$, whence $\lambda g \in B\left(\lambda f ; \lambda \varepsilon_{f}\right)$. Since on the other hand

$$
B\left(\lambda f ; \lambda \varepsilon_{f}\right) \cap \mathcal{A}=\lambda B\left(f ; \varepsilon_{f}\right) \cap \mathcal{A}=\lambda\left(B\left(f ; \varepsilon_{f}\right) \cap \mathcal{A}\right) \subseteq \lambda \mathcal{C}=\mathcal{C},
$$

we deduce that $\varepsilon_{\lambda f} \geq \lambda \varepsilon_{f}$, and as a consequence $\lambda g \in \widetilde{\mathcal{R}}$.

Consider a gamble $f \in \operatorname{posi}(\widetilde{\mathcal{R}}) \cap \mathcal{A}$. Then there are $f_{1}, \ldots, f_{n} \in \widetilde{\mathcal{R}}, \lambda_{1}, \ldots, \lambda_{n}>0$ such that $f=\sum_{i=1}^{n} \lambda_{i} f_{i}$. Note that since $\widetilde{\mathcal{R}}$ is a cone, we can assume without loss of generality that $\lambda_{i}=1$ for every $i=1, \ldots, n$. For every $i=1, \ldots, n$, there is some $g_{i} \in \mathcal{C}$ and some $h_{i} \in B\left(0 ; \varepsilon_{g_{i}}\right)$ such that $f_{i}=g_{i}+h_{i}$. 
Let us prove that there are $h_{i}^{\prime \prime} \in B\left(0 ; \varepsilon_{g_{i}}\right) \cap \mathcal{A}$ such that $f=\sum_{i=1}^{n}\left(g_{i}+h_{i}^{\prime \prime}\right)$. Since $g_{i}+h_{i}^{\prime \prime}$ will belong to $\widetilde{\mathcal{R}} \cap \mathcal{A}$, we will conclude that $f \in \operatorname{posi}(\widetilde{\mathcal{R}} \cap \mathcal{A})$, and the proof will be complete.

Note that we can work independently for each $\omega \in \Omega$; in addition, our construction of the gambles $h_{i}^{\prime \prime}$ shall not depend on the gambles $g_{i}$ above. Fix then $\omega \in \Omega$, and let $\mathcal{X}=\left\{x_{1}, \ldots, x_{k}\right\}$.

Consider the table

$$
\begin{array}{cccc}
h_{1}\left(\omega, x_{1}\right) & h_{2}\left(\omega, x_{1}\right) & \ldots & h_{n}\left(\omega, x_{1}\right) \\
h_{1}\left(\omega, x_{2}\right) & h_{2}\left(\omega, x_{2}\right) & \ldots & h_{n}\left(\omega, x_{2}\right) \\
\vdots & \vdots & \ldots & \vdots \\
h_{1}\left(\omega, x_{k}\right) & h_{2}\left(\omega, x_{k}\right) & \ldots & h_{n}\left(\omega, x_{k}\right) .
\end{array}
$$

By construction, the i-th column is bounded in $\left(-\varepsilon_{g_{i}}, \varepsilon_{g_{i}}\right)$, and the sum of all the elements of the table is equal to 0 , because $f \in \mathcal{A}$. Note, though, that the sum in each row is not necessarily equal to 0 .

Our goal then is to find a table

$$
\begin{array}{cccc}
h_{1}^{\prime \prime}\left(\omega, x_{1}\right) & h_{2}^{\prime \prime}\left(\omega, x_{1}\right) & \ldots & h_{n}^{\prime \prime}\left(\omega, x_{1}\right) \\
h_{1}^{\prime \prime}\left(\omega, x_{2}\right) & h_{2}^{\prime \prime}\left(\omega, x_{2}\right) & \ldots & h_{n}^{\prime \prime}\left(\omega, x_{2}\right) \\
\vdots & \vdots & \ldots & \vdots \\
h_{1}^{\prime \prime}\left(\omega, x_{k}\right) & h_{2}^{\prime \prime}\left(\omega, x_{k}\right) & \ldots & h_{n}^{\prime \prime}\left(\omega, x_{k}\right)
\end{array}
$$

such that:

$\circ(\forall i=1, \ldots, n) \sum_{j=1}^{k} h_{i}^{\prime \prime}\left(\omega, x_{j}\right)=0$.

$\circ(\forall i=1, \ldots, n)(\forall j=1, \ldots, k) h_{i}^{\prime \prime}\left(\omega, x_{j}\right) \in\left(-\varepsilon_{g_{i}}, \varepsilon_{g_{i}}\right)$.

$\circ(\forall j=1, \ldots, k) \sum_{i=1}^{n} h_{i}^{\prime \prime}\left(\omega, x_{j}\right)=\sum_{i=1}^{n} h_{i}\left(\omega, x_{j}\right)$.

The first of these requirements is done in order to guarantee that $h_{i}^{\prime \prime}$ belongs to $\mathcal{A}$; the second, to prove that $h_{i}^{\prime \prime}$ belongs to $B\left(0 ; \varepsilon_{g_{i}}\right)$; and the third, to guarantee that $f=\sum_{i=1}^{n}\left(g_{i}+h_{i}^{\prime \prime}\right)$. Then by repeating the process for every $\omega$ we end up with the gambles $h_{1}^{\prime \prime}, \ldots, h_{n}^{\prime \prime}$ required above.

Consider $j \in\{1, \ldots, n-1\}$, and let $f_{j}^{\prime}$ be a gamble on $\mathcal{X}$ whose supremum norm satisfies

$$
\left\|f_{j}^{\prime}\right\|<\sum_{i=j}^{n} \varepsilon_{g_{j}} .
$$

We shall show that there exists a gamble $h_{j}^{\prime}$ on $\mathcal{X}$ satisfying the following three conditions:

$$
\begin{aligned}
& \left\|f_{j}^{\prime}-h_{j}^{\prime}\right\|<\sum_{i=j+1}^{n} \varepsilon_{g_{i}} . \\
& \left\|h_{j}^{\prime}\right\|<\varepsilon_{g_{j} .} \\
& \sum_{x \in \mathcal{X}} h_{j}^{\prime}(x)=0 .
\end{aligned}
$$


Once we have established that it is possible to find the gamble $h_{j}^{\prime}$, we proceed as follows:

○ We consider the gamble $f_{1}^{\prime}$ on $\mathcal{X}$ given by $f_{1}^{\prime}(x):=\sum_{i=1}^{n} h_{i}(\omega, x)$. It satisfies $\left\|f_{1}^{\prime}\right\|<\sum_{i=1}^{n} \varepsilon_{g_{i}}$, so we find $h_{1}^{\prime}$ satisfying Eqs. (37)-(39) for $j=1$.

○ The gamble $f_{2}^{\prime}:=f_{1}^{\prime}-h_{1}^{\prime}$ satisfies $\left\|f_{2}^{\prime}\right\|<\sum_{i=2}^{n} \varepsilon_{g_{i}}$ because of Eq. (37), so we find $h_{2}^{\prime}$ satisfying Eqs. (37)-(39) for $j=2$.

○ We repeat the procedure for $f_{j}^{\prime}:=f_{j-1}^{\prime}-h_{j-1}^{\prime}$ for $j=3, \ldots, n-1$.

○ Finally, given $f_{n}^{\prime}:=f_{n-1}^{\prime}-h_{n-1}^{\prime}=f_{1}^{\prime}-h_{1}^{\prime}-\cdots-h_{n-1}^{\prime}$, we take $h_{n}^{\prime}:=f_{n}^{\prime}$. By (37), $\left\|h_{n}^{\prime}\right\|<\varepsilon_{g_{n}}$ and $f_{1}^{\prime}(x)=\sum_{i=1}^{n} h_{i}^{\prime}(x)$. Moreover,

$$
\sum_{x \in \mathcal{X}} h_{n}^{\prime}(x)=\sum_{x \in \mathcal{X}} f_{1}^{\prime}(x)-\sum_{i=1}^{n-1} \sum_{x \in \mathcal{X}} h_{i}^{\prime}(x)=0-\sum_{i=1}^{n-1} 0=0 .
$$

At that stage it suffices to make $h_{i}^{\prime \prime}\left(\omega, x_{j}\right)=h_{i}^{\prime}\left(x_{j}\right)$ for every $j=1, \ldots, k$ to obtain the gambles $h_{1}^{\prime \prime}, \ldots, h_{n}^{\prime \prime}$ we need.

So take $j \in\{1, \ldots, n-1\}$, a gamble $f_{j}^{\prime}$ with $\left\|f_{j}^{\prime}\right\|<\sum_{i=j}^{n} \varepsilon_{g_{i}}$ and let us establish the existence of a gamble $h_{j}^{\prime}$ satisfying conditions (37)-(39) above. Consider the partition of $\mathcal{X}$ given by:

$$
\begin{aligned}
& \mathcal{X}_{0}:=\left\{x: f_{j}^{\prime}(x)=0\right\}, \\
& \mathcal{X}_{1}:=\left\{x: f_{j}^{\prime}(x)>0, f_{j}^{\prime}(x) \geq \varepsilon_{g_{j}}\right\}, \\
& \mathcal{X}_{2}:=\left\{x: f_{j}^{\prime}(x)>0, f_{j}^{\prime}(x)<\varepsilon_{g_{j}}\right\}, \\
& \mathcal{X}_{3}:=\left\{x: f_{j}^{\prime}(x)<0, f_{j}^{\prime}(x) \leq-\varepsilon_{g_{j}}\right\}, \\
& \mathcal{X}_{4}:=\left\{x: f_{j}^{\prime}(x)<0, f_{j}^{\prime}(x)>-\varepsilon_{g_{j}}\right\} .
\end{aligned}
$$

We make $h_{j}^{\prime}(x):=f_{j}^{\prime}(x)=0$ for every $x \in \mathcal{X}_{0}$. Then $h_{j}^{\prime}$ satisfies Eqs. (37), (38) when it satisfies

$$
\begin{aligned}
& \left(\forall x \in \mathcal{X}_{1}\right) \quad h_{j}^{\prime}(x) \in\left(\max \left\{f_{j}^{\prime}(x)-\sum_{i=j+1}^{n} \varepsilon_{g_{i}}, 0\right\}, \varepsilon_{g_{j}}\right), \\
& \left(\forall x \in \mathcal{X}_{2}\right) \quad h_{j}^{\prime}(x) \in\left(\max \left\{f_{j}^{\prime}(x)-\sum_{i=j+1}^{n} \varepsilon_{g_{i}}, 0\right\}, f_{j}^{\prime}(x)\right], \\
& \left(\forall x \in \mathcal{X}_{3}\right) \quad h_{j}^{\prime}(x) \in\left(-\varepsilon_{g_{j}}, \max \left\{f_{j}^{\prime}(x)+\sum_{i=j+1}^{n} \varepsilon_{g_{i}}, 0\right\}\right), \\
& \left(\forall x \in \mathcal{X}_{4}\right) \quad h_{j}^{\prime}(x) \in\left[f_{j}^{\prime}(x), \max \left\{f_{j}^{\prime}(x)+\sum_{i=j+1}^{n} \varepsilon_{g_{i}}, 0\right\}\right) .
\end{aligned}
$$

Let us detail this for the case of $x \in \mathcal{X}_{1}$; the proof for the other cases is similar. First of all, since by construction $h_{j}^{\prime}(x)<\varepsilon_{g_{j}}<f_{j}^{\prime}(x)$ and also $0<h_{j}^{\prime}(x)$, we see that 
Eq. (38) is satisfied. With respect to (37), note that $\left|f_{j}^{\prime}(x)-h_{j}^{\prime}(x)\right|=f_{j}^{\prime}(x)-h_{j}^{\prime}(x)<$ $\sum_{i=j+1}^{n} \varepsilon_{g_{i}} \Leftrightarrow h_{j}^{\prime}(x)>f_{j}^{\prime}(x)-\sum_{i=j+1}^{n} \varepsilon_{g_{i}}$.

Now, it follows from interval arithmetic that $\sum_{x \in \mathcal{X}_{1} \cup \mathcal{X}_{2}} h_{j}^{\prime}(x)$ can take any value in

$$
\left(\sum_{x \in \mathcal{X}_{1} \cup \mathcal{X}_{2}} \max \left\{f_{j}^{\prime}(x)-\sum_{i=j+1}^{n} \varepsilon_{g_{i}}, 0\right\}, \sum_{x \in \mathcal{X}_{1}} \varepsilon_{g_{j}}+\sum_{x \in \mathcal{X}_{2}} f_{j}^{\prime}(x)\right)=:(C, D),
$$

and similarly $\sum_{x \in \mathcal{X}_{3} \cup \mathcal{X}_{4}} h_{j}^{\prime}(x)$ can take any value in

$$
\left(-\sum_{x \in \mathcal{X}_{3}} \varepsilon_{g_{j}}+\sum_{x \in \mathcal{X}_{4}} f_{j}^{\prime}(x), \sum_{x \in \mathcal{X}_{3} \cup \mathcal{X}_{4}} \max \left\{f_{j}^{\prime}(x)+\sum_{i=j+1}^{n} \varepsilon_{g_{i}}, 0\right\}\right)=:(E, F),
$$

and therefore that $\sum_{x \in \mathcal{X}} h_{j}^{\prime}(x)$ can take any value in the open interval $(C+E, D+F)$. Thus, in order to prove that it is possible to satisfy the constraints in Eqs. (40)-(43) and also $\sum_{x \in \mathcal{X}} h_{j}^{\prime}(x)=0$ (i.e., Eq. (39)), we are going to establish that $C+E<0<$ $D+F$.

Let us establish that $C+E<0$, or, in other words, that $C<-E$; the proof of $D+F>0$ is symmetrical.

Let $k:=\sum_{x \in \mathcal{X}_{1} \cup \mathcal{X}_{2}} f_{j}^{\prime}(x)>0$. Then, there exists some natural number $m$ such that $k \in\left[m \sum_{i=j}^{n} \varepsilon_{g_{i}},(m+1) \sum_{i=j}^{n} \varepsilon_{g_{i}}\right)$ (because the ratio $\frac{k}{\sum_{i=j}^{n} \varepsilon_{g_{i}}}$ is positive and so it must belong to an interval $[m, m+1)$ for some natural number $m$ ). Define $k^{\prime}:=$ $k-m \sum_{i=j}^{n} \varepsilon_{g_{i}} \in\left[0, \sum_{i=j}^{n} \varepsilon_{g_{i}}\right)$. Let us establish that $C$ is strictly bounded above by

$$
m \varepsilon_{g_{j}}+\max \left\{k^{\prime}-\sum_{i=j+1}^{n} \varepsilon_{g_{i}}, 0\right\} .
$$

Note that for every $x \in \mathcal{X}_{1} \cup \mathcal{X}_{2}$, it holds that $\max \left\{f_{j}^{\prime}(x)-\sum_{i=j+1}^{n} \varepsilon_{g_{i}}, 0\right\}>0$ only when $f_{j}^{\prime}(x) \geq \sum_{i=j+1}^{n} \varepsilon_{g_{i}}$, so if we define $B:=\left\{x: f_{j}^{\prime}(x) \geq \sum_{i=j+1}^{n} \varepsilon_{g_{i}}\right\}$ it holds that

$$
C= \begin{cases}\sum_{x \in B}\left(f_{j}^{\prime}(x)-\sum_{i=j+1}^{n} \varepsilon_{g_{i}}\right) & \text { if } B \neq \emptyset \\ 0 & \text { otherwise }\end{cases}
$$

This means that in order to maximize the value of $C$ we would like that $\sum_{x \in B} f_{j}^{\prime}(x)$ is as close to $k$ as possible. On the other hand, for a given value of $\sum_{x \in B} f^{\prime}(x)$, the outer sum in the right-hand side in (45) maximises when $B$ is as small as possible, which in turn implies that $f^{\prime}(x)$ is as large as possible for the elements of $B$. Putting these considerations together, we conclude that we maximize $C$ for a given value of $k$ when $B$ has $m+1$ elements: in $m$ of them we have $f_{j}^{\prime}(x)=\sum_{i=j}^{n} \varepsilon_{g_{i}}$ (and so $h_{j}^{\prime}=\varepsilon_{g_{j}}$ ), while in the other one $f_{j}^{\prime}(x)=k-m \sum_{i=j}^{n} \varepsilon_{g_{i}}=k^{\prime}\left(\right.$ and so $\left.h_{j}^{\prime}(x)=\max \left\{k^{\prime}-\sum_{i=j+1}^{n} \varepsilon_{g_{i}}, 0\right\}\right)$.

Thus $C$ is strictly bounded by Eq. (44); the strict inequality holds because by assumption $\left|f_{j}^{\prime}(x)\right|<\sum_{i=j}^{n} \varepsilon_{g_{i}}$ for every $x$, so we can only get arbitrarily close to the equalities mentioned in the previous phrase. 
Since $\sum_{x \in \mathcal{X}} f_{j}^{\prime}(x)=0$, it holds that $k=-\sum_{x \in \mathcal{X}_{3} \cup \mathcal{X}_{4}} f_{j}^{\prime}(x)$. Then $-E=\sum_{x \in \mathcal{X}_{3}} \varepsilon_{g_{j}}-$ $\sum_{x \in \mathcal{X}_{4}} f_{j}^{\prime}(x)$. For a lower bound on $-E$ we want this sum to be as far from $k$ as possible; this means that we want $\mathcal{X}_{3}$ to be as large as possible, and that for any $x \in \mathcal{X}_{3}$ we want to maximise $-f^{\prime}(x)-\varepsilon_{g_{j}}$. We do this when $-f^{\prime}(x)=\sum_{i=j}^{n} \varepsilon_{g_{i}}$ as often as possible (again $m$ times, taking into account the assumption of $k \in\left[m \sum_{i=j}^{n} \varepsilon_{g_{i}},(m+\right.$ 1) $\left.\sum_{i=j}^{n} \varepsilon_{g_{i}}\right)$ ); while for the remaining $k^{\prime}=k-m \sum_{i=j}^{n} \varepsilon_{g_{i}}$ we must allocate at least $\min \left\{k^{\prime}, \varepsilon_{g_{j}}\right\}$ (we allocate $\varepsilon_{g_{j}}$ if we can still be in $\mathcal{X}_{3}$ and $k^{\prime}$ when we must be in $\mathcal{X}_{4}$ ). These considerations imply that $-E$ is bounded below by

$$
m \varepsilon_{g_{j}}+\min \left\{k^{\prime}, \varepsilon_{g_{j}}\right\}
$$

with the same considerations about the strict inequality as above.

The result then follows by noting that the value in (44) is smaller than the value in (46). Indeed, there are two possible cases:

○ If $k^{\prime} \geq \varepsilon_{g_{j}}$, then

$$
\min \left\{k^{\prime}, \varepsilon_{g_{j}}\right\}=\varepsilon_{g_{j}} \geq \max \left\{k^{\prime}-\sum_{i=j+1}^{n} \varepsilon_{g_{i}}, 0\right\}, \text { because } k^{\prime} \leq \sum_{i=j}^{n} \varepsilon_{g_{i}}
$$

$\circ$ if $k^{\prime}<\varepsilon_{g_{j}}$, then we obtain

$$
\max \left\{k^{\prime}-\sum_{i=j+1}^{n} \varepsilon_{g_{i}}, 0\right\} \leq k^{\prime}=\min \left\{k^{\prime}, \varepsilon_{g_{j}}\right\} .
$$

We see then that $C+E<0$. In a similar way we can see that $D+F>0$. As a consequence, if we consider $h_{j}^{\prime}(x)$ in the intervals determined by Eqs. (40)-(43), the sum $\sum_{x \in \mathcal{X}} h_{j}^{\prime}(x)$ can take any value in the interval $(C+E, D+F)$, and in particular the value 0 . Thus, there is a gamble $h_{j}^{\prime}$ satisfying conditions (37)-(39). If we now apply this result in the manner described above, we end up with gambles $h_{1}^{\prime}, \ldots, h_{n}^{\prime}$ such that $f_{1}^{\prime}(x)=\sum_{i=1}^{n} h_{i}^{\prime}(x),\left\|h_{i}^{\prime}\right\|<\varepsilon_{g_{i}}$ and $\sum_{x \in \mathcal{X}} h_{i}^{\prime}(x)=0$ for all $i=1, \ldots, n$. Finally, repeating the process for every $\omega$ we obtain the gambles $h_{1}^{\prime \prime}, \ldots, h_{n}^{\prime \prime}$ required.

Proof of Proposition 26. We begin with the direct implication. From Lemma 36, if $\succ$ satisfies sA3 then for every $f \in \mathcal{C}$ there is some $\varepsilon_{f}>0$ such that $B\left(f ; \varepsilon_{f}\right) \cap \mathcal{A} \subseteq \mathcal{C}$. As a consequence, $\mathcal{C}$ is an open subset of $\mathcal{A}$.

Conversely, let $\mathcal{C}$ be an open convex cone in $\mathcal{A}$, and let $\succ$ be the coherent preference relation given by $p \succ q \Leftrightarrow p-q \in \mathcal{C}$. We show that $\succ$ satisfies sA3 by means of the characterisation in Proposition 23. Consider $f \in \mathcal{C}, g \in \mathcal{A}$. By Lemma 36, there is some $\varepsilon_{f}>$ 0 such that $B\left(f ; \varepsilon_{f}\right) \cap \mathcal{A} \subseteq \mathcal{C}$. In particular, given $\beta \in(0,1)$ such that $\|(1-\beta)(f-g)\|<\varepsilon_{f}$,

$$
\|f-(\beta f+(1-\beta) g)\|=\|(1-\beta)(f-g)\|<\varepsilon_{f} \Rightarrow \beta f+(1-\beta) g \in B\left(f ; \varepsilon_{f}\right) \cap \mathcal{A} .
$$

As a consequence, $\beta f+(1-\beta) g \in \mathcal{C}$ and applying Proposition 23 we conclude that $\succ$ is strongly Archimedean. 
Proof of Proposition 27. For the direct implication, it suffices to show that $\widetilde{\mathcal{R}}$ satisfies Eq. (25).

Consider a set $B$ and a gamble $f \in \widetilde{\mathcal{R}} \mid B$. Then there are $f_{1} \in \mathcal{R}_{1}\left|B, f_{2} \in \mathcal{R}_{2}\right| B$ such that $f=f_{1}+f_{2}$. Note that by construction $S\left(f_{1}\right), S\left(f_{2}\right), S(f) \subseteq B$ and also there is some $\varepsilon>0$ such that $B\left(f_{2}-\varepsilon\right) \in \mathcal{R}$. Let us prove that $I_{S(f)}\left(f-\frac{\varepsilon}{2}\right) \in \widetilde{\mathcal{R}} \mid B$. Since $S(f) \subseteq B$, it holds that, for any $\delta>0$,

$I_{S(f)}(f-\delta)=B(f-\delta)+\delta I_{B \backslash S(f)}=B\left(f_{1}+f_{2}-\delta\right)+\delta I_{B \backslash S(f)}=B\left(f_{2}-\delta\right)+B f_{1}+\delta I_{B \backslash S(f)} ;$

the gamble $B f_{1}+\delta I_{B \backslash S(f)}$ belongs to $\mathcal{R}_{1} \mid B$ for any $\delta>0$; moreover, $B\left(f_{2}-\delta\right)$ belongs to $\mathcal{R}_{2} \mid B$ for any $\delta \in(0, \varepsilon)$, and in particular for $\delta=\frac{\varepsilon}{2}$. As a consequence, $I_{S(f)}\left(f-\frac{\varepsilon}{2}\right) \in \widetilde{\mathcal{R}} \mid B$.

This implies that $\widetilde{\mathcal{R}} \mid B$ satisfies Eq. (25), and as a consequence so does $\widetilde{\mathcal{R}}$. Therefore, its natural extension $\mathcal{R}$ is a fully strictly desirable set of gambles.

As for the converse implication, by construction, $\widetilde{\mathcal{R}} \mid B \subseteq \mathcal{R}$ for every set $B$, whence $\mathcal{R}$ includes the natural extension of $\widetilde{\mathcal{R}}$. If $\mathcal{R}$ is a fully strictly desirable set, then it is the natural extension of a set $\mathcal{R}^{\prime}$ satisfying Eq. (25). Given a gamble $f \in \mathcal{R}^{\prime}$, it follows from (25) that $f \in \mathcal{R}_{2}|S(f) \subseteq \widetilde{\mathcal{R}}| S(f)$, and hence $\mathcal{R}^{\prime} \subseteq \widetilde{\mathcal{R}} \subseteq \mathcal{R}$. As a consequence, $\mathcal{R}$ is the natural extension of $\widetilde{\mathcal{R}}$.

Proof of Proposition 28. First of all, we are going to show that $\mathcal{R}$ is coherent. For this, it suffices to show that the set $\cup_{i \in I} \mathcal{R}_{2} \mid B_{i}$ avoids partial loss. Since for every $i \in I$ the set $\mathcal{R}_{2} \mid B_{i}$ is a convex cone, given that $\underline{P}_{i}\left(\cdot \mid B_{i}\right)$ is coherent, the set $\cup_{i \in I} \mathcal{R}_{2} \mid B_{i}$ incurs partial loss if and only if there is some finite $J \subseteq I$ and $g_{j} \in \mathcal{R}_{2} \mid B_{j}$ for every $j \in J$ such that $\sum_{j \in J} g_{j} \leq 0$. In that case,

$$
0 \geq \sum_{j \in J} g_{j}=\sum_{j \in J} B_{j}\left(f_{j}-\left(\underline{P}_{j}\left(f_{j} \mid B_{j}\right)-\varepsilon_{j}\right)\right),
$$

for some $f_{j} \in \mathcal{L}$ and some $\varepsilon_{j}>0$. As a consequence,

$$
\sup _{\omega \in \cup_{j \in J} B_{j}}\left[\sum_{j \in J} B_{j}\left(f_{j}-\underline{P}\left(f_{j} \mid B_{j}\right)\right)\right](\omega)<0,
$$

a contradiction with Eq. (27).

Let us show next that $\widetilde{\mathcal{R}} \mid B_{i}$ satisfies Eq. (25). Consider $f \in \widetilde{\mathcal{R}} \mid B_{i}$. Then there are $f_{1} \in$ $\mathcal{R}_{1}\left|B_{i}, f_{2} \in \mathcal{R}_{2}\right| B_{i}$ such that $f=f_{1}+f_{2}$. Note that by construction $S\left(f_{1}\right), S\left(f_{2}\right), S(f) \subseteq B_{i}$. Thus, for any $\delta>0$,

$$
\begin{aligned}
& I_{S(f)}(f-\delta)=B_{i}(f-\delta)+\delta I_{B_{i} \backslash S(f)}= \\
& B_{i}\left(f_{1}+f_{2}-\delta\right)+\delta I_{B_{i} \backslash S(f)}=B_{i}\left(f_{2}-\delta\right)+B_{i} f_{1}+\delta I_{B_{i} \backslash S(f)} ;
\end{aligned}
$$

the gamble $B_{i} f_{1}+\delta I_{B_{i} \backslash S(f)}$ belongs to $\mathcal{R}_{1} \mid B_{i}$ for any $\delta>0$; moreover, for $f_{2}$ there exists some $g_{2} \in \mathcal{L}$ and some $\varepsilon>0$ such that $f_{2}=B_{i}\left(g_{2}-\left(\underline{P}_{i}\left(g_{2} \mid B_{i}\right)-\varepsilon\right)\right)$, whence if we take $\delta \in(0, \varepsilon)$ we obtain that $B_{i}\left(f_{2}-\delta\right)=B_{i}\left(g_{2}-\left(\underline{P}_{i}\left(g_{2} \mid B_{i}\right)-(\varepsilon-\delta)\right)\right) \in \mathcal{R}_{2} \mid B_{i}$. As a consequence, $I_{S(f)}(f-\delta) \in \widetilde{\mathcal{R}} \mid B_{i}$.

We have obtained that the set $\widetilde{\mathcal{R}} \mid B_{i}$ satisfies Eq. (25) for all $i \in I$, and as a consequence so does the union $\cup_{i \in I} \widetilde{\mathcal{R}} \mid B_{i}$. Since $\mathcal{R}$ is the natural extension of $\cup_{i \in I} \widetilde{\mathcal{R}} \mid B_{i}$, we deduce that it is a fully strictly desirable set. 
Proof of Proposition 29. Assume that a set of gambles $\mathcal{R}^{\prime}$ satisfies (25), and let $\mathcal{R}$ be its natural extension. Then by definition of the natural extension, for every $f \in \mathcal{R}$ there are gambles $f_{1}, \ldots, f_{n} \in \mathcal{R}^{\prime}$ and positive real numbers $\lambda_{1}, \ldots, \lambda_{n}$ such that

$$
f=I_{S(f)} f \geq \sum_{j=1}^{n} \lambda_{j} f_{j} .
$$

By $(25)$, for every $j \in\{1, \ldots, n\}$ there is some $\varepsilon_{j}$ such that $I_{S\left(f_{j}\right)}\left(f_{j}-\varepsilon_{j}\right) \in \mathcal{R}^{\prime}$. Let

$$
\varepsilon:=\min \left\{\min _{j} \lambda_{j} \varepsilon_{j}, \min _{\omega \in S(f) \backslash \cup_{j} S\left(f_{j}\right)} f(\omega)\right\}>0 .
$$

Note that $f>0$ in $I_{S(f) \backslash \cup_{j} S\left(f_{j}\right)}$, provided that $S(f) \backslash \cup_{j} S\left(f_{j}\right) \neq \emptyset$, since $f \neq 0$ in $S(f)$, while $f \geq \sum_{j=1}^{n} \lambda_{j} f_{j}=0$ outside $\cup_{j} S\left(f_{j}\right)$; moreover, since $S(f)$ is finite we deduce that $\min _{\omega \in S(f) \backslash \cup_{j} S\left(f_{j}\right)} f(\omega)>0$. Taking into account that $\lambda_{j} \varepsilon_{j}>0$ for all $j$, we conclude that indeed $\varepsilon>0$. Moreover, we deduce also that

$$
I_{S(f) \backslash \cup_{j} S\left(f_{j}\right)}(f-\varepsilon) \geq 0
$$

to prove this, it is enough to reconsider that $f>0$ in $S(f) \backslash \cup_{j} S\left(f_{j}\right)$, and that $\varepsilon \leq$ $\min _{\omega \in S(f) \backslash \cup_{j} S\left(f_{j}\right)} f(\omega)$.

Now,

$$
\begin{aligned}
I_{S(f)}(f-\varepsilon) & \geq\left(\sum_{j=1}^{n} \lambda_{j} f_{j}\right)-\varepsilon I_{S(f)} \geq \sum_{j=1}^{n} \lambda_{j} I_{S\left(f_{j}\right)} f_{j}-\varepsilon I_{\cup_{j} S\left(f_{j}\right)}-\varepsilon I_{S(f) \backslash \cup_{j} S\left(f_{j}\right)} \\
& \geq \sum_{j=1}^{n}\left(\lambda_{j} I_{S\left(f_{j}\right)} f_{j}-\varepsilon I_{S\left(f_{j}\right)}\right)-\varepsilon I_{S(f) \backslash \cup_{j} S\left(f_{j}\right)} \\
& \geq \sum_{j=1}^{n} \lambda_{j} I_{S\left(f_{j}\right)}\left(f_{j}-\varepsilon_{j}\right)-\varepsilon I_{S(f) \backslash \cup_{j} S\left(f_{j}\right)},
\end{aligned}
$$

where in the last passage we have used that $\varepsilon \leq \min _{j} \lambda_{j} \varepsilon_{j}$.

Consider two cases:

o If $\omega \in S(f) \backslash \cup_{j} S\left(f_{j}\right)$, then $I_{S(f)}(f-\varepsilon) \geq 0=\sum_{j=1}^{n} \lambda_{j} I_{S\left(f_{j}\right)}\left(f_{j}-\varepsilon_{j}\right)$, where the inequality follows from (47).

○ If $\omega \in \cup_{j} S\left(f_{j}\right)$, then $I_{S(f)}(f-\varepsilon) \geq \sum_{j=1}^{n} \lambda_{j} I_{S\left(f_{j}\right)}\left(f_{j}-\varepsilon_{j}\right)$, as it follows from (48).

As a consequence, $I_{S(f)}(f-\varepsilon) \geq \sum_{j=1}^{n} \lambda_{j} I_{S\left(f_{j}\right)}\left(f_{j}-\varepsilon_{j}\right)$, and since $I_{S\left(f_{j}\right)}\left(f_{j}-\varepsilon_{j}\right) \in \mathcal{R}^{\prime}$, we deduce that $I_{S(f)}(f-\varepsilon)$ belongs to $\mathcal{R}$. Thus, $\mathcal{R}$ also satisfies $(25)$.

We conclude that $\mathcal{R}$ is fully strictly desirable if and only if it satisfies (25).

\section{Appendix C. List of Symbols and Their Relations}

Table 1 below provides a summary list of the main symbols used in the paper with their meaning.

Figure 1 displays the most relevant relations between them: an arrow from symbol A to $\mathrm{B}$ means that $\mathrm{B}$ can be derived or constructed from A. The number in parentheses on the arrows point to the equations where the relations are detailed. 


\begin{tabular}{c|c} 
Symbol & Meaning \\
\hline$\Omega$ & Space of possibilities \\
$\mathcal{X}$ & Space of outcomes \\
$\mathcal{X}_{z}$ & Space of outcomes, with a worst outcome \\
$\mathcal{H}$ & Set of horse lotteries \\
$\mathcal{H}_{z}$ & Set of horse lotteries, with a worst outcome \\
$\mathcal{A}$ & Scaled differences of horse lotteries \\
$\mathcal{C}$ & Scaled differences of preferences \\
$\mathcal{B}$ & Generic partition of $\Omega$ \\
$\mathcal{L}$ & Space of gambles \\
$\mathcal{L}^{+}$ & Positive gambles \\
$\mathcal{R}$ & A set of desirable gambles \\
$\mathcal{R} \mid B$ & A set of gambles conditional on a set $B$ \\
$\mathcal{R}\rfloor B$ & An equivalent representation of $\mathcal{R} \mid B$ \\
$\mathcal{R} \mid \Omega$ & A set of gambles conditional on partition $\Omega$ of $\Omega$ \\
$\mathcal{R} \Omega$ & Marginal set of gambles on $\Omega$ \\
$\mathcal{R}_{\Omega}^{\prime}$ & An equivalent representation of $\mathcal{R}_{\Omega}$ \\
$\mathcal{R}$ & Marginal extension of $\mathcal{R}$ \\
$\succ$ & Preference relation \\
$\triangleright$ & Objective preference relation \\
$\underline{P}$ & Coherent lower prevision \\
$\mathcal{P}$ & Coherent upper prevision \\
$\mathcal{M}(\underline{P})$ & Credal set associated with $\underline{P}$
\end{tabular}

Table 1: Main symbols used in the paper with their meaning. 


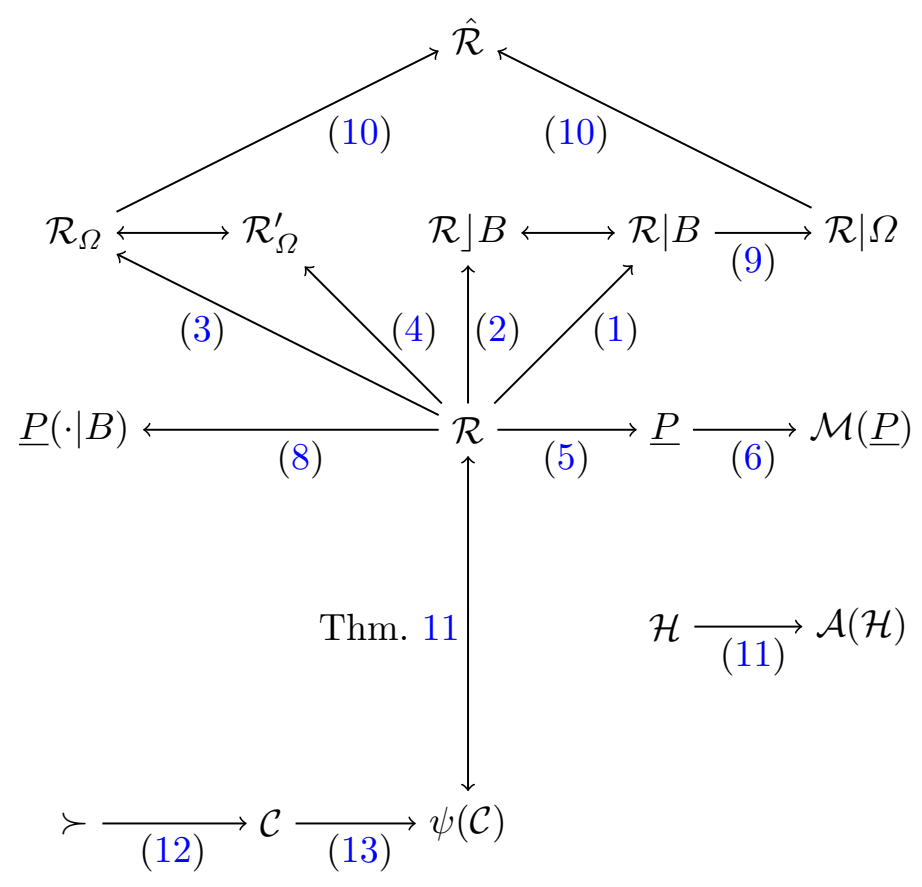

Figure 1: Relevant relations between the main concepts.

\section{References}

Allais, M. (1953). Le comportement de l'homme rationnel devant le risque: critique des postulats et axiomes de l'école Américaine. Econometrica, 21 (4), 503-546.

Anscombe, F. J., \& Aumann, R. J. (1963). A definition of subjective probability. The Annals of Mathematical Statistics, 34, 199-2005.

Artzner, P., Delbaen, F., Eber, J.-M., \& Heath, D. (1999). Coherent measures of risk. Mathematical Finance, 9, 203-228.

Augustin, T., Coolen, F., de Cooman, G., \& Troffaes, M. (Eds.). (2014). Introduction to Imprecise Probabilities. Wiley.

Aumann, R. J. (1962). Utility theory without the completeness axiom. Econometrica, 30, $445-462$.

Bewley, T. (2002). Knightian decision theory: part I. Decisions in economics and finance, 25, 79-110. Reprint of Discussion Paper 807, Cowles Foundation, 1986.

Cheridito, P. (2013). Lecture Notes in Convex Analysis. Princeton University. https: //people.math.ethz.ch/ patrickc/CA2013.pdf.

Coletti, G., \& Scozzafava, R. (2002). Probabilistic logic in a coherent setting. Kluwer.

Corani, G., \& Zaffalon, M. (2008). Learning reliable classifiers from small or incomplete data sets: the naive credal classifier 2. Journal of Machine Learning Research, 9, $581-621$. 
Couso, I., \& Moral, S. (2011). Sets of desirable gambles: Conditioning, representation, and precise probabilities. International Journal of Approximate Reasoning, 52, 1034-1055.

Cozman, F. G. (2012). Sets of probability distributions, independence, and convexity. Synthese, $186(2), 577-600$.

De Bock, J., \& de Cooman, G. (2014). An efficient algorithm for estimating state sequences in imprecise hidden Markov models. Journal of Artificial Intelligence Research, 50, 189-233.

De Bock, J., \& de Cooman, G. (2015a). Conditioning, updating and lower probability zero. International Journal of Approximate Reasoning, 67, 1-36.

De Bock, J., \& de Cooman, G. (2015b). Credal networks under epistemic irrelevance: The sets of desirable gambles approach. International Journal of Approximate Reasoning, 56, 178-207.

De Cooman, G., \& Hermans, F. (2008). Imprecise probability trees: bridging two theories of imprecise probability. Artificial Intelligence, 172(11), 1400-1427.

De Cooman, G., \& Miranda, E. (2009). Forward irrelevance. Journal of Statistical Planning and Inference, $139(2), 256-276$.

De Cooman, G., \& Miranda, E. (2012). Irrelevance and independence for sets of desirable gambles. Journal of Artificial Intelligence Research, 45, 601-640.

De Cooman, G., Miranda, E., \& Zaffalon, M. (2011). Independent natural extension. Artificial Intelligence, 175(12-13), 1911-1950.

De Cooman, G., \& Quaeghebeur, E. (2012). Exchangeability and sets of desirable gambles. International Journal of Approximate Reasoning, 53, 363-395. Special issue in honour of Henry E. Kyburg, Jr.

De Finetti, B. (1937). La prévision: ses lois logiques, ses sources subjectives. Annales de l'Institut Henri Poincaré, 7, 1-68. English translation in (Kyburg Jr. \& Smokler, 1964).

Del Amo, A. G., \& Ríos Insua, D. (2002). A note on an open problem in the foundations of statistics. RACSAM, 96(1), 55-61.

Drèze, J. H. (1971). Market allocation under uncertainty. European Economic Review, 2(2), $133-165$.

Dubins, L. E. (1975). Finitely additive conditional probabilities, conglomerability and disintegrations. The Annals of Probability, 3, 88-99.

Dubois, D., Fargier, H., \& Perny, P. (2003). Qualitative decision theory with preference relations and comparative uncertainty: an axiomatic approach. Artificial Intelligence, 148(1-2), 219-260.

Dubra, J., Maccheroni, F., \& Ok, E. (2004). Expected utility theory without the completeness axiom. Journal of Economic Theory, 115, 118-133.

Galaabaatar, T., \& Karni, E. (2013). Subjective expected utility with incomplete preferences. Econometrica, 81(1), 255-284. 
Ghirardato, P., \& Siniscalchi, M. (2012). Ambiguity in the small and in the large. Econometrica, 80(6), 2827-2847.

Gilboa, I., Maccheroni, F., Marinacci, M., \& Schmeidler, D. (2010). Objective and subjective rationality in a multiple prior model. Econometrica, 78, 755-770.

Gilboa, I., \& Schmeidler, D. (1989). Maxmin expected utility with a non-unique prior. Journal of Mathematical Economics, 18, 141-153.

Kikuti, D., Cozman, F. G., \& Filho, R. S. (2011). Sequential decision making with partially ordered preferences. Artificial Intelligence, 175(7-8), 1346-1365.

Kyburg Jr., H. E., \& Smokler, H. E. (Eds.). (1964). Studies in Subjective Probability. Wiley, New York. Second edition (with new material) 1980.

Miranda, E. (2008). A survey of the theory of coherent lower previsions. International Journal of Approximate Reasoning, 48(2), 628-658.

Miranda, E., \& de Cooman, G. (2007). Marginal extension in the theory of coherent lower previsions. International Journal of Approximate Reasoning, 46(1), 188-225.

Miranda, E., \& Zaffalon, M. (2010). Notes on desirability and conditional lower previsions. Annals of Mathematics and Artificial Intelligence, 60(3-4), 251-309.

Miranda, E., \& Zaffalon, M. (2015). Independent products in infinite spaces. Journal of Mathematical Analysis and Applications, 425(1), 460-488.

Miranda, E., Zaffalon, M., \& de Cooman, G. (2012). Conglomerable natural extension. International Journal of Approximate Reasoning, 53(8), 1200-1227.

Miranda, E., \& Zaffalon, M. (2016). Conformity and independence with coherent lower previsions. International Journal of Approximate Reasoning, 78, 125-137.

Moral, S. (2005). Epistemic irrelevance on sets of desirable gambles. Annals of Mathematics and Artificial Intelligence, 45, 197-214.

Nau, R. (2006). The shape of incomplete preferences. The Annals of Statistics, 34(5), $2430-2448$.

Nau, R. (2008). Phd seminar on choice theory. Course notes. https://faculty.fuqua. duke.edu/ rnau/choice/choice02.pdf.

Nau, R. (2016). Arbitrage and Rational Choice. In preparation.

Ok, E., Ortoleva, P., \& Riella, G. (2012). Incomplete preferences under uncertainty: indecisiveness in beliefs versus tastes. Econometrica, 80(4), 1791-1808.

Pedersen, A. P. (2014). Comparative expectations. Studia Logica, 102(4), 811-848.

Quaeghebeur, E., de Cooman, G., \& Hermans, F. (2015). Accept \& reject statement-based uncertainty models. International Journal of Approximate Reasoning, 57, 69-102.

Rockafellar, R. T. (1970). Convex Analysis. Princeton University Press, Princeton, NJ.

Savage, L. J. (1954). The Foundations of Statistics. Wiley, New York.

Seidenfeld, T., Schervish, M. J., \& Kadane, J. B. (1990). Decisions without ordering. In Sieg, W. (Ed.), Acting and reflecting, Vol. 211 of Synthese Library, pp. 143-170. Kluwer, Dordrecht. 
Seidenfeld, T., Schervish, M. J., \& Kadane, J. B. (1995). A representation of partially ordered preferences. The Annals of Statistics, 23, 2168-2217. Reprinted in (Seidenfeld, Schervish, \& Kadane, 1999), pp. 69-129.

Seidenfeld, T., Schervish, M. J., \& Kadane, J. B. (1999). Rethinking the Foundations of Statistics. Cambridge University Press, Cambridge.

Shimony, A. (1955). Coherence and the axioms of confirmation. The Journal of Symbolic Logic, 20(1), 1-28.

Smith, C. A. B. (1961). Consistency in statistical inference and decision. Journal of the Royal Statistical Society, Series A, 23, 1-37.

Von Neumann, J., \& Morgestern, O. (1947). Theory of games and economic behaviour. Princeton University Press.

Walley, P. (1991). Statistical Reasoning with Imprecise Probabilities. Chapman and Hall, London.

Williams, P. M. (1975). Notes on conditional previsions. Tech. rep., School of Mathematical and Physical Science, University of Sussex, UK. Reprinted in (Williams, 2007).

Williams, P. M. (2007). Notes on conditional previsions. International Journal of Approximate Reasoning, 44, 366-383. Revised journal version of (Williams, 1975).

Zaffalon, M., \& Miranda, E. (2013). Probability and time. Artificial Intelligence, 198(1), $1-51$.

Zaffalon, M., \& Miranda, E. (2015). Desirability and the birth of incomplete preferences. CoRR, abs/1506.00529. http://arxiv.org/abs/1506.00529. 